\title{
Elder-Rule-Staircodes for Augmented Metric Spaces
}

\author{
Chen Cai ${ }^{* 1}$, Woojin $\mathrm{Kim}^{\dagger 3}$, Facundo Mémoli ${ }^{\ddagger 1,2}$, and Yusu Wang ${ }^{\S 1}$ \\ ${ }^{1}$ Department of Computer Science and Engineering, The Ohio State \\ University. \\ ${ }^{2}$ Department of Mathematics, The Ohio State University. \\ ${ }^{3}$ Department of Mathematics, Duke University.
}

July 14, 2020

\begin{abstract}
An augmented metric space is a metric space $\left(X, d_{X}\right)$ equipped with a function $f_{X}$ : $X \rightarrow \mathbb{R}$. This type of data arises commonly in practice, e.g, a point cloud $X$ in $\mathbb{R}^{d}$ where each point $x \in X$ has a density function value $f_{X}(x)$ associated to it. An augmented metric space $\left(X, d_{X}, f_{X}\right)$ naturally gives rise to a 2 -parameter filtration $\mathcal{K}$. However, the resulting 2-parameter persistent homology $\mathrm{H}_{\bullet}(\mathcal{K})$ could still be of wild representation type, and may not have simple indecomposables. In this paper, motivated by the elder-rule for the zeroth homology of 1-parameter filtration, we propose a barcode-like summary, called the elder-rule-staircode, as a way to encode $\mathrm{H}_{0}(\mathcal{K})$. Specifically, if $n=$ $|X|$, the elder-rule-staircode consists of $n$ number of staircase-like blocks in the plane. We show that if $\mathrm{H}_{0}(\mathcal{K})$ is interval decomposable, then the barcode of $\mathrm{H}_{0}(\mathcal{K})$ is equal to the elder-rule-staircode. Furthermore, regardless of the interval decomposability, the fibered barcode, the dimension function (a.k.a. the Hilbert function), and the graded Betti numbers of $\mathrm{H}_{0}(\mathcal{K})$ can all be efficiently computed once the elder-rule-staircode is given. Finally, we develop and implement an efficient algorithm to compute the elderrule-staircode in $O\left(n^{2} \log n\right)$ time, which can be improved to $O\left(n^{2} \alpha(n)\right)$ if $X$ is from a fixed dimensional Euclidean space $\mathbb{R}^{d}$, where $\alpha(n)$ is the inverse Ackermann function.
\end{abstract}

\section{Introduction}

An augmented metric space is a metric space $\left(X, d_{X}\right)$ equipped with a function $f_{X}: X \rightarrow \mathbb{R}$ $[4,11,17]$. This type of data arises commonly in practice: e.g, a point cloud $X$ in $\mathbb{R}^{d}$ where each point has a density function value $f_{X}$ associated to it. Studying hierarchical clustering

*cai.507@osu.edu

${ }^{\dagger}$ woojin.kim205@duke.edu

${ }^{\ddagger}$ memoli@math.osu.edu

$\S_{\text {yusu@cse.ohio-state.edu }}$ 
methods induced in this setting has attracted much attention starting with [11] and more recently with $[4,8,35]$. Another example is where $X=V$ equals to the vertex set of a graph $G=(V, E), d_{X}$ represents certain graph-induced metric on $X$ (e.g, the diffusion distance induced by $G$ ), and $f_{X}$ is some descriptor function (e.g, discrete Ricci curvature) at graph nodes. This graph setting occurs often in practice for graph analysis applications, where $G$ can be viewed as a skeleton of a hidden domain. When summarizing or characterizing $G$, one wishes to take into consideration both the metric structure of this domain and node attributes. Given that persistence-based summaries from only the edge weights or from only node attributes have already shown promise in graph classification (e.g, [7, 13, 27, 43]), it would be highly desirable to incorporate (potentially more informative) summaries encoding both types of information to tackle such tasks. In brief, we wish to develop topological invariants induced from such augmented metric spaces.

On the other hand, an augmented metric space naturally gives rise to a 2-parameter filtration (by filtering both via $f_{X}$ and via distance $d_{X}$; see Definition 3.2). However, while a standard (1-parameter) filtration and its induced persistence module has persistence diagram as a complete discrete invariant, multi-parameter persistence modules do not have such complete discrete invariant $[9,20]$. The 2-parameter persistence module induced from an augmented metric space may still be of wild representation type, and may not have simple indecomposables [4]. Instead, several recent works consider informative (but not necessarily complete) invariants for multiparameter persistence modules [23, 26, 28, 33, 36, 37, 42]. In particular, RIVET [33] provides an interactive visualization of barcodes associated to 1dimensional slices of an input 2-parameter persistence module $M$, which are called the fibered barcode. For implementing the interactive aspect, RIVET makes efficient use of graded Betti numbers of $M$, another invariant of the 2-parameter persistence module $M$.

Our contributions. We propose a barcode-like summary, called the elder-rule-staircode, as a way to encode the zeroth homology of the 2-parameter filtration induced by a finite augmented metric space. Specifically, given a finite $\mathcal{X}=\left(X, d_{X}, f_{X}\right)$, its elder-rule-staircode consists of $n=|X|$ number of staircase-like blocks of $O(n)$ descriptive complexity in the plane. The development of the elder-rule-staircode is motivated by the elder-rule behind the construction of persistence pairing for a 1-parameter filtration [24]. For the 1-parameter case, barcodes [44] can be obtained by the decomposition of persistence modules in the realm of commutative algebra, or equivalently, by applying the elder-rule which is flavored with combinatorics or order theory. As we describe in Section 4, our elder-rule-staircodes are obtained by adapting the elder-rule for treegrams arisen from 1-parameter filtration.

Interestingly, we show that our elder-rule-staircode encodes much of topological information of the 2-parameter filtration $\mathcal{K}$ induced by $\mathcal{X}$. In particular, the fibered barcodes, the fibered treegrams, and the graded Betti numbers associated to $\mathrm{H}_{0}(\mathcal{K})$ can all be efficiently computed from the elder-rule-staircodes (see Theorems 3.7, 4.13 and 5.4). Furthermore, if $\mathrm{H}_{0}(\mathcal{K})$ is interval decomposable, then the interval indecomposables appearing in its decomposition correspond exactly to its staircode (see Theorem 4.16). This implies that testing the interval decomposability of $\mathrm{H}_{0}(\mathcal{K})$ is reduced to testing isomorphism of two given persistence modules [6] (see Remark 4.17). We also provide sufficient conditions on $\mathcal{X}$ which ensure the interval decomposability of $\mathrm{H}_{0}(\mathcal{K})$ (see Theorem 4.10 and Corollary 4.11). Therefore, 
to explore exotic isomorphism types of indecomposable summands of $\mathrm{H}_{0}(\mathcal{K}$ ) (a question of interest considered in [4]), it suffices to restrict our attention to augmented metric spaces which do not satisfy these conditions.

Finally, in Section 6, we show that the elder-rule-staircode can be computed in $O\left(n^{2} \log n\right)$ time for a finite augmented metric space $\left(X, d_{X}, f_{X}\right)$ where $n=|X|$, and $O\left(n^{2} \alpha(n)\right)$ time if $X$ is from a fixed dimensional Euclidean space and $d_{X}$ is Euclidean distance. We have software to compute elder-rule-staircodes and to explore / retrieve information such as fibered barcodes interactively, which is available at https://github.com/Chen-Cai-OSU/ ER-staircode.

More on related work. The elder-rule is an underlying principle for extracting the persistence diagram from a persistence module induced by a nested family of simplicial complexes [24, Chapter 7]. Recently this principle has come into the spotlight again for generalizing persistence diagrams [28, 36, 39] and for addressing inverse problems in TDA [21]. An algorithm for testing interval decomposability of multiparameter persistence modules has been studied [1]. A method to approximate 2-parameter persistence modules by interval-decomposable persistence modules has been proposed [2].

The software RIVET and work of [34] can also be used to recover fibered barcodes and graded Betti numbers. However, for the special case of zeroth 2-parameter persistence modules induced from augmented metric spaces, our elder-rule-staircodes are simpler and more efficient to achieve these goals: In particular, given an augmented metric space containing $n$ points, the algorithm of [34] computes the graded Betti numbers in $\Omega\left(n^{3}\right)$ time, while it takes $O\left(n^{2} \log n\right)$ time using elder-rule-staircode via Theorem 6.1. For zeroth fibered barcodes, RIVET takes $O\left(n^{8}\right)$ time to compute a data structure of size $O\left(n^{6}\right)$ so as to support efficient query time of $O\left(\log n+\left|B^{L}\right|\right)$ where $\left|B^{L}\right|$ is the size of the fibered barcode $B^{L}$ for a particular line $L$ of positive slope. Our algorithm computes elder-rule-staircode of size $O\left(n^{2}\right)$ in $O\left(n^{2} \log n\right)$ time, after which $B^{L}$ can be computed in $O\left(\left|B^{L}\right| \log n\right)$ time for any query line $L$. See Section 6.2 for more detailed comparison. However, it is important to note that RIVET allows much broader inputs and can work beyond zeroth homology.

Outline. In Section 2 we review the definitions of persistence modules, barcodes, and graded Betti numbers. In Section 3 we introduce a 2-parameter filtration $\mathcal{K}$ induced by an augmented metric space $\mathcal{X}$ and define the elder-rule-staircode of $\mathcal{X}$. In Section 4 we show that the elder-rule-staircode recovers the fibered barcode of $\mathrm{H}_{0}(\mathcal{K})$. We also prove that, if $\mathrm{H}_{0}(\mathcal{K})$ is interval decomposable, then the set of indecomposables corresponds exactly to the staircode. In Section 5 we show that the elder-rule-staircode recovers the graded Betti numbers of $\mathrm{H}_{0}(\mathcal{K})$. In Section 6 we develop and implement an efficient algorithm to compute the elder-rule-staircode. In Section 7 we discuss open problems. For readability we have relegated some proofs to an appendix.

Acknowledgements. The authors thank to the anonymous reviewers who made a number of helpful comments to improve the paper. Also, CC and WK thank Cheng Xin for helpful discussions. This work is supported by NSF grants DMS-1723003, CCF-1740761, DMS1547357, and IIS-1815697. 


\section{Preliminaries}

In Section 2.1 we review the definitions of persistence modules and their barcodes. In Section 2.2 we review the notion of graded Betti number of a persistence module.

\subsection{Persistence modules and their decompositions}

First we briefly review the definition of persistence modules. Let $\mathbb{P}$ be a poset. We regard $\mathbb{P}$ as the category that has elements of $\mathbb{P}$ as objects. Also, for any $\mathbf{a}, \mathbf{b} \in \mathbb{P}$, there exists a unique morphism $\mathbf{a} \rightarrow \mathbf{b}$ if and only if $\mathbf{a} \leq \mathbf{b}$. For $d \in \mathbb{N}$, let $\mathbb{Z}^{d}$ be the set of $d$-tuples of integers equipped with the partial order defined as $\left(a_{1}, a_{2}, \ldots, a_{d}\right) \leq\left(b_{1}, b_{2}, \ldots, b_{d}\right)$ if and only if $a_{i} \leq b_{i}$ for each $i=1,2, \ldots, d$. The poset structure on $\mathbb{R}^{d}$ is defined in the same way.

We fix a certain field $\mathbb{F}$ and every vector space in this paper is over $\mathbb{F}$. Let Vec denote the category of finite dimensional vector spaces over $\mathbb{F}$.

A ( $\mathbb{P}$-indexed) persistence module is a functor $M: \mathbb{P} \rightarrow$ Vec. In other words, to each $\mathbf{a} \in \mathbb{P}$, a vector space $M(\mathbf{a})$ is associated, and to each pair $\mathbf{a} \leq \mathbf{b}$ in $\mathbb{P}$, a linear map $\varphi_{M}(\mathbf{a}, \mathbf{b}): M(\mathbf{a}) \rightarrow M(\mathbf{b})$ is associated. When $\mathbb{P}=\mathbb{R}^{d}$ or $\mathbb{Z}^{d}, M$ is said to be a d-parameter persistence module. A morphism between $M, N: \mathbb{P} \rightarrow$ Vec is a natural transformation $f: M \rightarrow N$ between $M$ and $N$. That is, $f$ is a collection $\left\{f_{\mathbf{a}}\right\}_{\mathbf{a} \in \mathbb{P}}$ of linear maps such that for every pair $\mathbf{a} \leq \mathbf{b}$ in $\mathbb{P}$, the following diagram commutes:

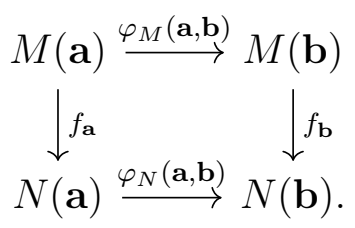

Two persistence modules $M$ and $N$ are isomorphic, denoted by $M \cong N$, if there exists a natural transformation $\left\{f_{\mathbf{a}}\right\}_{\mathbf{a} \in \mathbb{P}}$ from $M$ to $N$ where each $f_{\mathbf{a}}$ is an isomorphism.

We now review the standard definition of barcodes, following notation from [5].

Definition 2.1 (Intervals). Let $\mathbb{P}$ be a poset. An interval $\mathcal{J}$ of $\mathbb{P}$ is a subset $\mathcal{J} \subset \mathbb{P}$ s.t.: (1) $\mathcal{J}$ is non-empty. (2) If $\mathbf{a}, \mathbf{b} \in \mathcal{J}$ and $\mathbf{a} \leq \mathbf{c} \leq \mathbf{b}$, then $\mathbf{c} \in \mathcal{J}$. (3) For any $\mathbf{a}, \mathbf{b} \in \mathcal{J}$, there is a sequence $\mathbf{a}=\mathbf{a}_{0}, \mathbf{a}_{1}, \cdots, \mathbf{a}_{l}=\mathbf{b}$ of elements of $\mathcal{J}$ with $\mathbf{a}_{i}$ and $\mathbf{a}_{i+1}$ comparable for $0 \leq i \leq l-1$.

For $\mathcal{J}$ an interval of $\mathbb{P}$, the interval module $I^{\mathcal{J}}: \mathbb{P} \rightarrow$ Vec is defined as

$$
I^{\mathcal{J}}(\mathbf{a})=\left\{\begin{array}{ll}
\mathbb{F} & \text { if } \mathbf{a} \in \mathcal{J}, \\
0 & \text { otherwise }
\end{array} \quad \varphi_{I \mathcal{J}}(\mathbf{a}, \mathbf{b})= \begin{cases}\operatorname{id}_{\mathbb{F}} & \text { if } \mathbf{a}, \mathbf{b} \in \mathcal{J}, \mathbf{a} \leq \mathbf{b} \\
0 & \text { otherwise }\end{cases}\right.
$$

Recall that a multiset is a collection of objects (called elements) in which elements may occur more than once, and the number of instances of an element is its multiplicity.

Definition 2.2 (Interval decomposability and barcodes). A functor $M: \mathbb{P} \rightarrow$ Vec is interval decomposable if there exists a multiset barc $(M)$ of intervals (Definition 2.1) of $\mathbb{P}$ such that $M \cong \bigoplus_{\mathcal{J} \in \operatorname{barc}(M)} I^{\mathcal{J}}$. We call $\mathbf{b a r c}(M)$ the barcode of $M$. 
By the theorem of Azumaya-Krull-Remak-Schmidt [3], such a decomposition is unique up to a permutation of the terms in the direct sum. Therefore, the multiset $\operatorname{barc}(M)$ is unique if $M$ is interval decomposable. For $d=1$, any $M: \mathbb{R}^{d}\left(\right.$ or $\left.\mathbb{Z}^{d}\right) \rightarrow$ Vec is interval decomposable and thus $\operatorname{barc}(M)$ exists. However, for $d \geq 2, M$ may not be interval decomposable.

\subsection{Graded Betti numbers}

Persistence module as a module over a polynomial ring. In Section 2.1 we defined $d$-parameter persistence modules as Vec-valued functors over the posets $\mathbb{Z}^{d}$ or $\mathbb{R}^{d}$, and morphisms between them as natural transformations. Definitions below are equivalent to those definitions [9, Theorem 1], and allow us to define the graded Betti numbers of persistence modules. We mostly adopt notation in [23, 34].

Let $\mathbb{F}\left[t_{1}, t_{2}, \ldots, t_{d}\right]$ be the polynomial ring in the $d$-variables $t_{1}, t_{2}, \ldots, t_{d}$. To ease notation, for $\mathbf{n}:=\left(n_{1}, n_{2}, \ldots, n_{d}\right) \in \mathbb{Z}_{>0}^{d}$, the monomial $t_{1}^{n_{1}} t_{2}^{n_{2}} \ldots t_{d}^{n_{d}} \in \mathbb{F}\left[t_{1}, t_{2}, \ldots, t_{n}\right]$ will be written as $\mathbf{x}^{\mathbf{n}}$. A $d$-parameter persistence module $M: \mathbb{Z}^{d} \rightarrow$ Vec is an $\mathbb{F}\left[t_{1}, t_{2}, \ldots, t_{d}\right]$-module $M$ with a direct sum decomposition as an $\mathbb{F}$-vector space $M \cong \bigoplus_{\mathbf{a} \in \mathbb{Z}^{d}} M_{\mathbf{a}}$ such that the action of $\mathbb{F}\left[t_{1}, t_{2}, \ldots, t_{d}\right]$ on $M$ is uniquely specified as follows: for all $\mathbf{a}=\left(a_{1}, a_{2}, \ldots, a_{d}\right) \in \mathbb{Z}^{d}$ and $v \in M_{\mathbf{a}}$, and for all $\mathbf{n}=\left(n_{1}, n_{2}, \ldots, n_{d}\right) \in \mathbb{Z}_{\geq 0}^{d}$, and for all $c \in k$,

$$
\left(c \cdot \mathbf{x}^{\mathbf{n}}\right) \cdot v:=c \cdot \varphi_{M}(\mathbf{a}, \mathbf{a}+\mathbf{n})(v) .
$$

Let $M$ and $N$ be any two persistence modules. A morphism $f: M \rightarrow N$ is the module homomorphism such that $f\left(M_{\mathbf{a}}\right) \subseteq N_{\mathbf{a}}$ for all $\mathbf{a} \in \mathbb{Z}^{d}$. Since our interests are in studying finite aug-MSs, we will restrict ourselves to finite persistence modules [9] - the $k$-th homology of a filtration of a finite simplicial complex for some $k \in \mathbb{Z}_{\geq 0}$ - in what follows.

The kernel, image, and cokernel of $f$ are analogously defined to those of a linear map between vector spaces. The kernel of $f$ is defined as the submodule $\operatorname{ker}(f):=\bigoplus_{\mathbf{a} \in \mathbb{Z}^{d}} \operatorname{ker}\left(f_{\mathbf{a}}\right)$ of $M$. The image of $f$ is defined as the submodule $\operatorname{im}(f):=\bigoplus_{\mathbf{a} \in \mathbb{Z}^{d}} \operatorname{im}\left(f_{\mathbf{a}}\right)$ of $N$. The cokernel of $f$ is defined as coker $(f):=\bigoplus_{\mathbf{a} \in \mathbb{Z}^{d}}\left(N_{\mathbf{a}} / \operatorname{im}\left(f_{\mathbf{a}}\right)\right)$.

Graded Betti numbers. We briefly review the concept of graded Betti numbers [9, 30, 33, 34, 40, 44]. Since our interests are in studying finite aug-MSs, we restrict ourselves to finite persistence modules - the $k$-th homology of a filtration of a finite simplicial complex for some $k \in \mathbb{Z}_{\geq 0}[9]$.

Fix $\mathbf{a} \in \mathbb{Z}^{d}$. By $Q^{\mathbf{a}}: \mathbb{Z}^{d} \rightarrow$ Vec, we denote the persistence module defined as

$$
Q_{\mathbf{x}}^{\mathbf{a}}=\left\{\begin{array}{ll}
\mathbb{F}, & \text { if } \mathbf{a} \leq \mathbf{x} \\
0, & \text { otherwise }
\end{array} \quad \varphi_{Q^{\mathbf{a}}}(\mathbf{x}, \mathbf{y})= \begin{cases}\mathrm{id}_{\mathbb{F}}, & \text { if } \mathbf{a} \leq \mathbf{x} \\
0, & \text { otherwise }\end{cases}\right.
$$

Any $F: \mathbb{Z}^{d} \rightarrow$ Vec is said to be free if there exists a multiset $\mathcal{A}$ of elements of $\mathbb{Z}^{2}$ such that $F \cong \bigoplus_{\mathbf{a} \in \mathcal{A}} Q^{\mathbf{a}}$. For simplicity, we will refer to free persistence modules as free modules. Let $M$ be a persistence module. An element $m \in M_{\mathbf{a}}$ for some $\mathbf{a} \in \mathbb{Z}^{d}$ is called a homogeneous element of $M$. In this case, we write $\operatorname{gr}(m)=\mathbf{a}$. Let $F$ be a free module. A basis $B$ of $F$ is defined as a minimal homogeneous set of generators of $F$. There can exist two bases $B$ and $B^{\prime}$ of $F$ (analogous to the fact that a vector space can have multiple bases). However, the number of elements at each grade $\mathbf{a} \in \mathbb{Z}^{d}$ in a basis of $F$ is an isomorphism invariant. 
For a finite $M$, let $I M$ denote the submodule of $M$ generated by the images of all linear maps $\varphi_{M}(\mathbf{a}, \mathbf{b})$, with $\mathbf{a}<\mathbf{b}$ in $\mathbb{Z}^{2}$. Assume that there is a chain of modules

$$
F^{\bullet}: \cdots \stackrel{\partial_{3}}{\longrightarrow} F^{2} \stackrel{\partial_{2}}{\longrightarrow} F^{1} \stackrel{\partial_{1}}{\longrightarrow} F^{0} \stackrel{\partial_{0}}{\longrightarrow} M \stackrel{0\left(=: \partial_{-1}\right)}{\longrightarrow} 0
$$

such that (1) each $F^{i}$ is a free module, and $(2) \operatorname{im}\left(\partial^{i}\right)=\operatorname{ker}\left(\partial^{i-1}\right), i=0,1,2, \cdots$. Then we call $F^{\bullet}$ a resolution of $M$. The condition (2) is referred to as exactness of $F^{\bullet}$. We call the resolution $F^{\bullet}$ minimal if $\operatorname{im}\left(\partial^{i}\right) \subseteq I F^{i-1}$ for $i=1,2, \cdots$. It is a standard fact that a minimal resolution of $M$ always exists and is unique up to isomorphism [40, Chapter I].

Definition 2.3 (Graded Betti numbers). Let $M: \mathbb{Z}^{d} \rightarrow$ Vec be finite. Assume that a minimal free resolution of $M$ is $F^{\bullet}$ in (1). For $i \in \mathbb{Z}_{\geq 0}$, the $i$-th graded Betti number $\beta_{i}^{M}: \mathbb{Z}^{d} \rightarrow \mathbb{Z}_{\geq 0}$ is defined as $\beta_{i}^{M}(\mathbf{a})=$ (number of elements at grade $\mathbf{a}$ in any basis of $F^{i}$ ).

Remark 2.4. (i) Note that if $M \cong N_{1} \bigoplus N_{2}$, then $\beta_{i}^{M}=\beta_{i}^{N_{1}}+\beta_{i}^{N_{2}}$. This is a key fact to define the persistent graded Betti numbers introduced in [23].

(ii) $\beta_{i}^{M}: \mathbb{Z}^{d} \rightarrow \mathbb{Z}_{\geq 0}$ is the zero function for every integer $i>d$ [25, Theorem 1.13].

(iii) Definition 2.3 is not in the exactly same form as those in the literature such as [9, 30, 33]. However, by Nakayama's lemma [40, Lemma 2.11] all those are equivalent, as already noted in [34, Section 2.3].

For any $M: \mathbb{Z}^{d} \rightarrow$ Vec, the dimension function $\operatorname{dm}(M): \mathbb{Z}^{d} \rightarrow \mathbb{Z}_{\geq 0}$ of $M$ is defined as $\mathbf{a} \mapsto \operatorname{dim} M_{\mathbf{a}}$. The graded Betti numbers of $M$ recover $\operatorname{dm}(M)$ :

Theorem 2.5 ([34, Proposition 2.3]). Let $M: \mathbb{Z}^{d} \rightarrow$ Vec be a finite persistence module. For all $\mathbf{a} \in \mathbb{Z}^{d}$,

$$
\operatorname{dm}(M)(\mathbf{a})=\sum_{\mathbf{x} \leq \mathbf{a}} \sum_{i=0}^{d}(-1)^{i} \beta_{i}^{M}(\mathbf{x})
$$

\section{$3 \quad$ Elder-rule-staircodes for augmented metric spaces}

Rips bifiltration for an aug-MS. Let $\left(X, d_{X}\right)$ be a metric space. For $\varepsilon \in \mathbb{R}$, the Rips complex $\mathcal{R}_{\varepsilon}\left(X, d_{X}\right)$ is the abstract simplicial complex defined as

$$
\mathcal{R}_{\varepsilon}\left(X, d_{X}\right)=\left\{A \subseteq X: \text { for all } x, x^{\prime} \in A, d_{X}\left(x, x^{\prime}\right) \leq \varepsilon\right\} .
$$

Let Simp be the category of abstract simplicial complexes and simplicial maps. The Rips filtration is the functor $\mathcal{R}_{\bullet}\left(X, d_{X}\right): \mathbb{R} \rightarrow$ Simp defined as

$$
\varepsilon \mapsto \mathcal{R}_{\varepsilon}\left(X, d_{X}\right) \text {, and } \varepsilon \leq \varepsilon^{\prime} \mapsto \mathcal{R}_{\varepsilon}\left(X, d_{X}\right) \hookrightarrow \mathcal{R}_{\varepsilon^{\prime}}\left(X, d_{X}\right)
$$

Definition 3.1 (Augmented metric spaces). Let $\left(X, d_{X}\right)$ be a metric space and $f_{X}: X \rightarrow \mathbb{R}$ a function. We call the triple $\mathcal{X}=\left(X, d_{X}, f_{X}\right)$ an augmented metric space (abbrev. aug-MS).

We say that $\mathcal{X}$ is injective if $f_{X}: X \rightarrow \mathbb{R}$ is an injective function. 
Throughout this paper, every (augmented) metric space will be assumed to be finite. Let $\mathcal{X}=\left(X, d_{X}, f_{X}\right)$ be an aug-MS. For $\sigma \in \mathbb{R}$, let $X_{\sigma}$ denote the sublevel set $f_{X}^{-1}(-\infty, \sigma] \subseteq X$. Let $\left(X_{\sigma}, d_{X}\right)$ denote the restriction of the metric space $\left(X, d_{X}\right)$ to the subset $X_{\sigma} \subseteq X$. Similarly, $\left(X_{\sigma}, d_{X}, f_{X}\right)$ is the aug-MS obtained by restricting $d_{X}$ to $X_{\sigma} \times X_{\sigma}$ and $f_{X}$ to $X_{\sigma}$. The following 2-parameter filtration is considered in $[4,11,12]$ in the context of filtered single linkage hierarchical clustering or filtered persistent homology:

Definition 3.2 (Rips bifiltration of an aug-MS). Let $\mathcal{X}=\left(X, d_{X}, f_{X}\right)$ be an aug-MS. We define the Rips bifiltration $\mathcal{R}_{\bullet}^{\text {bi }}(\mathcal{X}): \mathbb{R}^{2} \rightarrow \operatorname{Simp}$ of $\mathcal{X}$ as $(\varepsilon, \sigma) \mapsto \mathcal{R}_{\varepsilon}\left(X_{\sigma}, d_{X}\right)$.

By applying the $k$-th simplicial homology functor to the Rips bifiltration $\mathcal{R}_{\bullet}^{\text {bi }}(\mathcal{X})$, we obtain the persistence module $M:=\mathrm{H}_{k}\left(\mathcal{R}_{\bullet}^{\mathrm{bi}}(\mathcal{X})\right): \mathbb{R}^{2} \rightarrow$ Vec. Let $\mathcal{L}$ denote the set of all lines of (strictly) positive slopes in $\mathbb{R}^{2}$. Given $L \in \mathcal{L}$, the restriction $\left.M\right|_{L}: L \rightarrow$ Vec can be decomposed into the unique direct sum of interval modules over $L$ and thus we have the barcode $\operatorname{barc}\left(\left.M\right|_{L}\right)$ of $\left.M\right|_{L}$. The $k$-th fibered barcode of $\mathcal{X}$ refers to the $\mathcal{L}$-parametrized collection $\left\{\operatorname{barc}\left(\left.M\right|_{L}\right)\right\}_{L \in \mathcal{L}}[14,31,33]$.

Elder-rule-staircode for an aug-MS. Let $\left(X, d_{X}\right)$ be a finite metric space. For $\varepsilon \in$ $[0, \infty)$, an $\varepsilon$-chain between $x, x^{\prime} \in X$ stands for a sequence $x=x_{1}, x_{2}, \ldots, x_{\ell}=x^{\prime}$ of points in $X$ such that $d_{X}\left(x_{i}, x_{i+1}\right) \leq \varepsilon$ for $i=1, \ldots, \ell-1$. Now given $\mathcal{X}=\left(X, d_{X}, f_{X}\right)$ and $\sigma \in \mathbb{R}_{\geq 0}$, consider a point $x \in X_{\sigma}$. Then for any $\varepsilon \geq 0$, set $[x]_{(\sigma, \varepsilon)}$ as the collection of all points $x^{\prime} \in X_{\sigma}$ that can be connected to $x$ through an $\varepsilon$-chain in $X_{\sigma}$.

The function $f_{X}: X \rightarrow \mathbb{R}$ induces an order on $X$ : consider any two $x, x^{\prime} \in X$. If $f_{X}(x)<f_{X}\left(x^{\prime}\right)$, then we say that $x$ is older than $x^{\prime}$.

Definition 3.3 (Elder-rule-staircode for an aug-MS). Let $\mathcal{X}=\left(X, d_{X}, f_{X}\right)$ be an injective aug-MS. For each $x \in X$, we define its staircode as:

$$
I_{x}:=\left\{(\sigma, \varepsilon) \in \mathbb{R}^{2}: x \in X_{\sigma} \text { and } x \text { is the oldest in }[x]_{(\sigma, \varepsilon)}\right\}
$$

The collection $\mathcal{I}_{\mathcal{X}}:=\left\{I_{x}\right\}_{x \in X}$ is called the elder-rule-staircode (ER-staircode for short) of $\mathcal{X}$.

See Figure 1 for an example. The relationship between the ER-staircode and the classic elder-rule will become clear in Section 4.1.

Definition 3.4. An interval I of $\mathbb{R}^{2}$ (Definition 2.1) is a staircase interval (or simply staircase) if there exists $\left(\sigma_{0}, \varepsilon_{0}\right) \in I$ such that $\left(\sigma_{0}, \varepsilon_{0}\right) \leq(\sigma, \varepsilon)$ for all $(\sigma, \varepsilon) \in I$, and $I$ is not bounded in the direction of $\sigma$-axis (see Figure 3).

It turns out that each $I_{x} \in \mathcal{I}_{\mathcal{X}}$ is a staircase interval:

Proposition 3.5. Each $I_{x}$ in Definition 3.3 is a staircase interval (proof in Appendix A). 


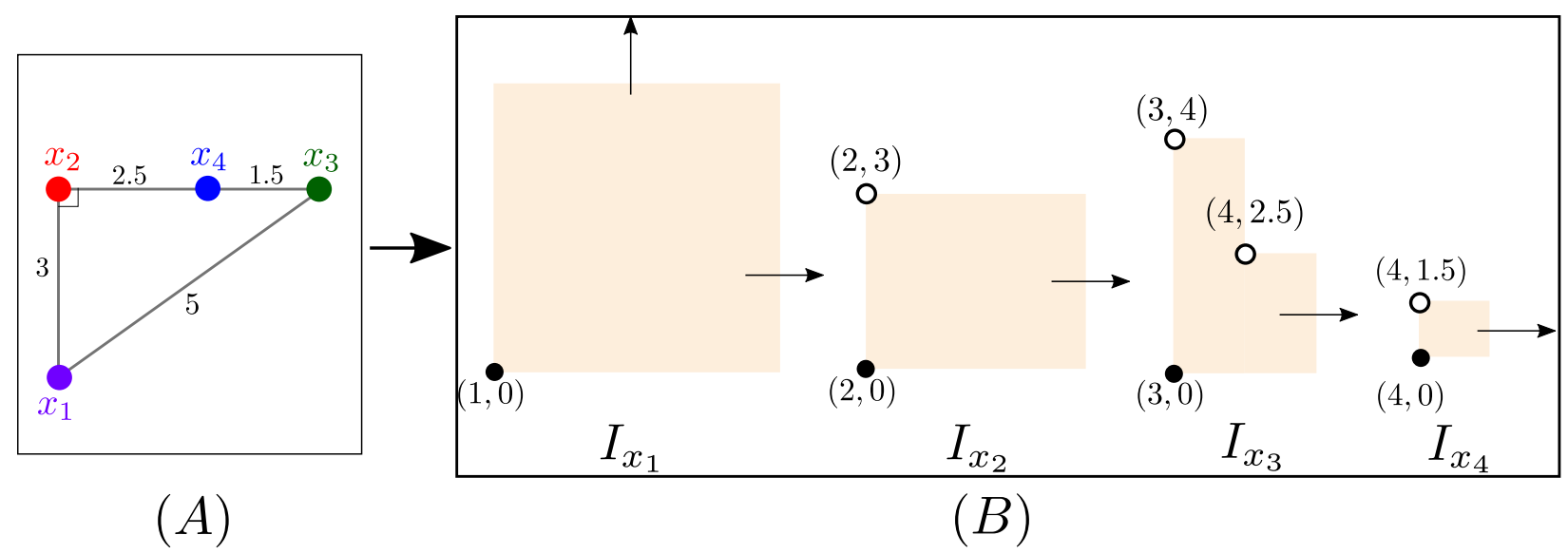

Figure 1: (A) Consider the triangle with edge lengths 3,4 and 5. Consider the aug-MS $\mathcal{X}=\left(X, d_{X}, f_{X}\right)$ where $X:=\left\{x_{1}, x_{2}, x_{3}, x_{4}\right\}, d_{X}$ is the Euclidean metric on the plane, and $f_{X}$ is given as $f_{X}\left(x_{i}\right)=i$ for $i=1,2,3,4$. (B) The ER-staircode of $\mathcal{X}$.

Staircodes for non-injective case. Even if $f_{X}$ is not injective, we still have the concept of the ER-staircode. Consider an aug-MS $\mathcal{X}=\left(X, d_{X}, f_{X}\right)$ such that $f_{X}$ is not injective. To induce the ER-staircode of $\mathcal{X}$, we pick any order on $X$ which is compatible with $f_{X}$ : An order $<$ on $X$ is compatible with $f_{X}$ if $f_{X}(x)<f_{X}\left(x^{\prime}\right)$ implies $x<x^{\prime}$ for all $x, x^{\prime} \in X$. Now we define $\mathcal{I}_{\mathcal{X}}^{<}=\left\{\left\{I_{x}^{<}: x \in X\right\}\right.$ where

$$
I_{x}^{<}:=\left\{(\sigma, \varepsilon) \in \mathbb{R}^{2}: x \in X_{\sigma} \text { and } x=\min \left([x]_{(\sigma, \varepsilon)},<\right)\right\}
$$

(we use double-curly-brackets $\{\{-\}$ to denote multisets). Regardless of the choice of $<$, the collection $\mathcal{I}_{\mathcal{X}}^{<}=\left\{I_{x}^{<}: x \in X\right\}$ satisfies all properties and theorems we prove later. Hence, for any possible compatible order $<$ we will refer to $\mathcal{I}_{\mathcal{X}}^{<}$as an ER-staircode of $\mathcal{X}$.

Example 3.6 (Constant function case). Let $\left(X, d_{X}\right)$ be a metric space of $n$ points. Then, the barcode of $\mathrm{H}_{0}\left(\mathcal{R} \bullet\left(X, d_{X}\right)\right): \mathbb{R} \rightarrow$ Vec consists of $n$ intervals $J_{i}, i=1, \ldots, n$. Let $\mathcal{X}=$ $\left(X, d_{X}, f_{X}\right)$ be the aug-MS where $f_{X}$ is constant at $c \in \mathbb{R}$. Then, all possible total orders on $X$ are compatible with $f_{X}$ and all induce the same ER-staircode $\mathcal{I}_{\mathcal{X}}=\left\{[c, \infty) \times J_{i}: i=1, \ldots, n\right\}$.

In contrast to Example 3.6, different orders on $X$ in general induce different ER-staircodes of $\mathcal{X}=\left(X, d_{X}, f_{X}\right)$; see Example 3.8. Therefore, a single ER-staircode of $\mathcal{X}$ is not necessarily an invariant of $\mathcal{X}$, whereas the collection of all possible ER-staircodes of $\mathcal{X}$ can be seen so (see item 4 in Section 7). This collection, however, is not a complete invariant of $\mathcal{X}$ by the following reasoning: It is not difficult to find two non-isometric metric spaces $\left(X, d_{X}\right)$ and $\left(Y, d_{Y}\right)$ such that $\mathrm{H}_{0}\left(\mathcal{R} \bullet\left(X, d_{X}\right)\right)$ and $\mathrm{H}_{0}\left(\mathcal{R} \bullet\left(Y, d_{Y}\right)\right)$ have the same barcode. Let $f_{X}: X \rightarrow \mathbb{R}$ and $f_{Y}: Y \rightarrow \mathbb{R}$ be constant at $c \in \mathbb{R}$. Then, by Example 3.6, all the ER-staircodes of $\left(X, d_{X}, f_{X}\right)$ and $\left(Y, d_{Y}, f_{Y}\right)$ (induced by all possible total orders on $X$ and $Y$ ) are the same (see item 5 in Section 7).

We can recover the zeroth fibered barcode of an aug-MS $\mathcal{X}$ from its ER-staircode: Computation of ER-staircode and query time for fibered barcode are given in Theorem 6.1. 


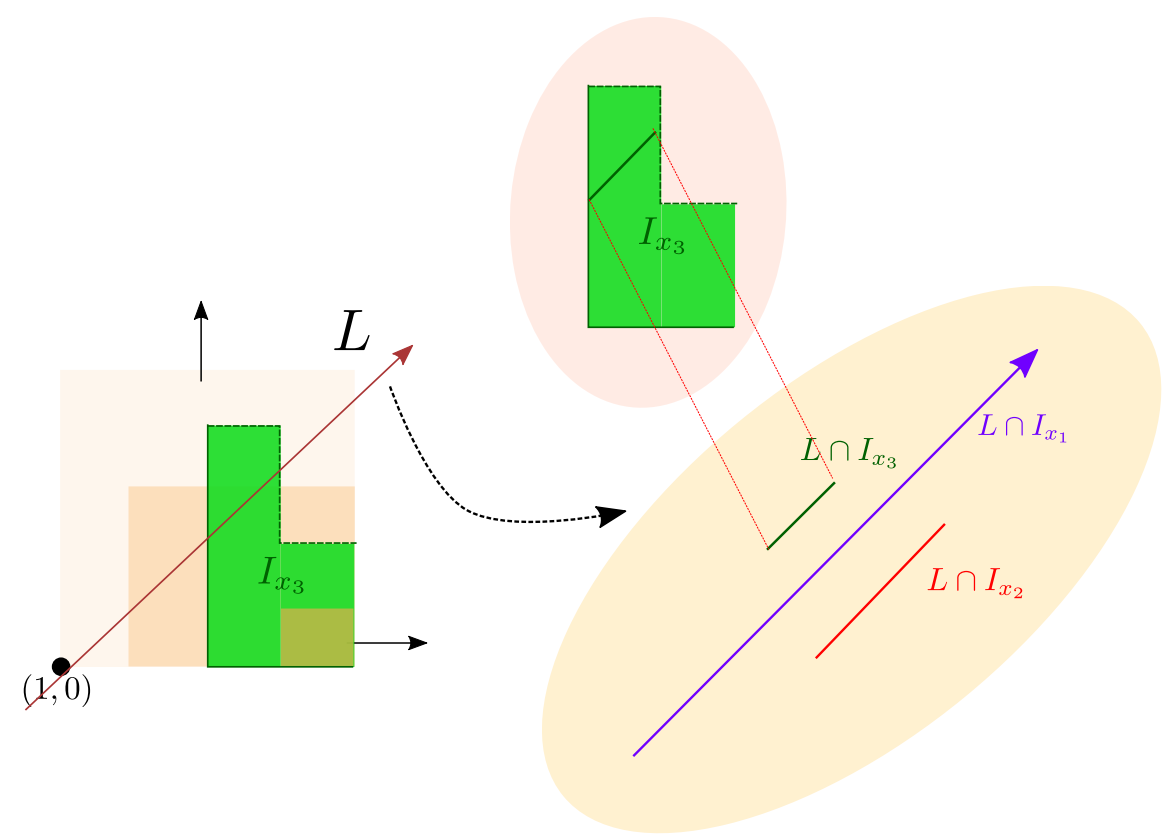

Figure 2: Left: The stack of $I_{x_{i}}, i=1,2,3,4$ from Figure 1 and a line $L \in \mathcal{L}$. Right: The barcode of $\left.M\right|_{L}$. Since $L$ does not intersect $I_{x_{4}}$, only three intervals of $L \subset \mathbb{R}^{2}$ appear in the barcode.

Theorem 3.7. Let $\mathcal{X}$ be an aug-MS and let $M:=\mathrm{H}_{0}\left(\mathcal{R}_{\bullet}^{\mathrm{bi}}(\mathcal{X})\right)$. Let $\mathcal{I}_{\mathcal{X}}=\left\{\left\{I_{x}: x \in X\right\}\right.$ be an ER-staircode of $\mathcal{X}$. For each $L \in \mathcal{L}$, the barcode $\operatorname{barc}\left(\left.M\right|_{L}\right)$ coincides with the multiset $\left\{\left\{\cap I_{x}: x \in X\right\}\right.$ (up to removal of empty sets, see Figure 2), (proof in Section 4.1).

Example 3.8. If an aug-MS is not injective, then there can be different ER-staircodes w.r.t. different compatible orders. However, each of them will still be valid to produce the fibered barcodes. For example, let $\left(X, d_{X}\right)$ be the metric space in Figure 1 (A). Define $g_{X}: X \rightarrow \mathbb{R}$ by sending $x_{1}, x_{2}, x_{3}, x_{4}$ to $1,2,2,4$, respectively. Two orders $\left(x_{1}<x_{2}<x_{3}<x_{4}\right)$ and $\left(x_{1}<^{\prime} x_{3}<^{\prime} x_{2}<^{\prime} x_{4}\right)$ are compatible with $g_{X}$. Consider the two ER-staircodes $\mathcal{I}_{\mathcal{X}}^{<}=$ $\left\{\left\{I_{x_{i}}^{<}: i=1,2,3,4\right\}\right\}$ and $\mathcal{I}_{\mathcal{X}}^{<^{\prime}}=\left\{\left\{I_{x_{i}}^{<^{\prime}}: i=1,2,3,4\right\}\right\}$. While $I_{x_{i}}^{<}=I_{x_{i}}^{<^{\prime}}$ for $i=1,4$, the equality does not hold for $i=2,3$. However, both $\mathcal{I}_{\mathcal{X}}^{<}$and $\mathcal{I}_{\mathcal{X}}^{<}$satisfy the statement in Theorem 3.7. See Figure 3.

We will close this section with some definitions that will be useful later. Let $I$ be a staircase interval of $\mathbb{R}^{2}$. We define the three types of corner points as in Figure 3. Roughly speaking, for each staircase $I_{x}$, type-0 is the left-bottom point; type- 1 corners are those where the boundary transitions from a vertical segment to a horizontal one, while type- 2 are those transitions from a horizontal one to vertical one (precise descriptions are given in Definition A.1 of Appendix A).

Given a staircase interval $I$, for each $j=0,1,2$ we define the function $\gamma_{j}(I): \mathbb{R}^{2} \rightarrow \mathbb{Z}_{\geq 0}$ as

$$
\gamma_{j}(I)(\mathbf{a})= \begin{cases}1, & \mathbf{a} \text { is a } j \text {-th type corner point of } I \\ 0, & \text { otherwise. }\end{cases}
$$

Elder-rule feature functions defined below will be useful in the later chapters. 


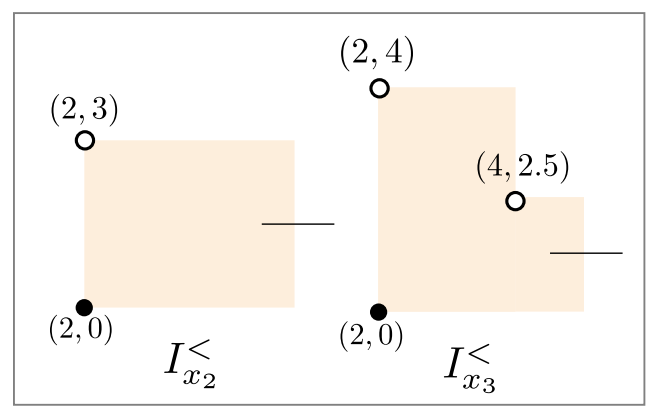

$(A)$

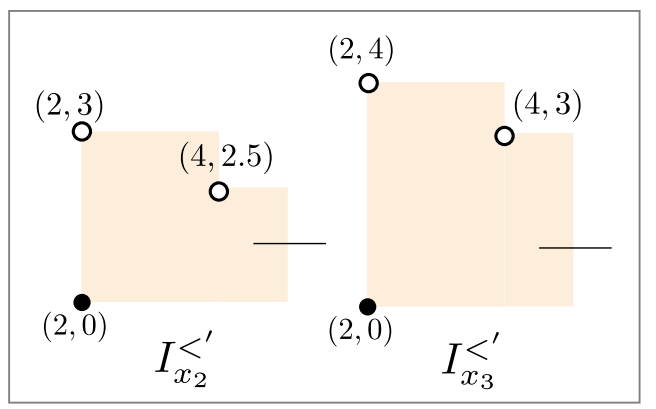

$(B)$

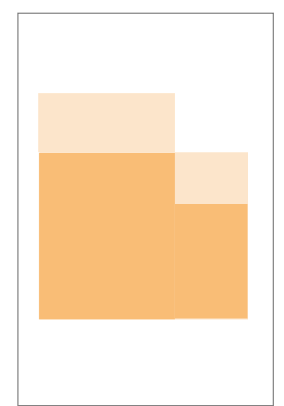

$(C)$

Figure 3: Illustration for Example 3.8: (A) $I_{x_{2}}^{<}$and $I_{x_{3}}^{<}$. (B) $I_{x_{2}}^{<^{\prime}}$ and $I_{x_{3}}^{<^{\prime}}$. (C) Stack of $I_{x_{2}}^{<}$and $I_{x_{3}}^{<}$. Stack of $I_{x_{2}}^{<^{\prime}}$ and $I_{x_{3}}^{<^{\prime}}$ look the same. Observe that for any $L \in \mathcal{L},\left\{\left\{L \cap I_{x_{2}}^{<}, L \cap I_{x_{3}}^{<}\right\}\right\}=$ $\left\{\left\{L \cap I_{x_{2}}^{<^{\prime}}, L \cap I_{x_{3}}^{<^{\prime}}\right\}\right\}$.

Definition 3.9 (Elder-rule feature functions). Let $\mathcal{X}$ be an aug-MS and let $I_{\mathcal{X}}=\left\{\left\{I_{x}: x \in X\right\}\right.$ be an ER-staircode of $\mathcal{X}$. For $j=0,1,2$, we define the $j$-th elder-rule feature function as the sum $\gamma_{j}^{\mathcal{X}}=\sum_{x \in X} \gamma_{j}\left(I_{x}\right)$.

Remark 3.10. It is not hard to check that $\gamma_{j}(I)$ in (4) is equal to the $j$-th graded Betti number of the interval module $\mathbb{R}^{2} \rightarrow$ Vec supported by $I$ (Definition 2.3). Thus $\gamma_{j}^{\mathcal{X}}=$ $\sum_{x \in X} \beta_{j}^{I^{I x}}$.

\section{Decorated elder-rule-staircodes and treegrams}

In Section 4.1 we prove Theorem 3.7 and introduce bipersistence treegrams to encode multiscale clustering information of aug-MSs. In Section 4.2 we show that an "enriched" ERstaircode of an aug-MS $\mathcal{X}$ can recover the so-called fibered treegram of $\mathcal{X}$, i.e. 1-dimensional slices of the aforementioned bipersistence treegram. Also, we identify a sufficient condition on $\mathcal{X}$ for its ER-staircode to be the barcode of the 2-parameter persistence module $\mathrm{H}_{0}\left(\mathcal{R}_{\bullet}^{\mathrm{bi}}(\mathcal{X})\right)$. In Section 4.3 we show that if $\mathrm{H}_{0}\left(\mathcal{R}_{\bullet}^{\mathrm{bi}}(\mathcal{X})\right)$ is interval decomposable, then its barcode is equal to the ER-staircode of $\mathcal{X}$. Also, we stratify the collection of aug-MSs $\mathcal{X}$ according to the complexity of the indecomposable summands of $\mathrm{H}_{0}\left(\mathcal{R}_{\bullet}^{\mathrm{bi}}(\mathcal{X})\right)$.

\subsection{Bipersistence treegrams}

Partitions and sub-partitions. Let $X$ be a non-empty finite set. We will call any partition $P$ of a subset $X^{\prime}$ of $X$ a sub-partition of $X$. In this case we call $X^{\prime}$ the underlying set of $P$. A partition of the empty set is defined as the empty set. By $\operatorname{Subpart}(X)$, we denote the set of all sub-partitions of $X$, i.e. $\operatorname{Subpart}(X):=\left\{P: \exists X^{\prime} \subseteq X, P\right.$ is a partition of $\left.X^{\prime}\right\}$. We refer to elements of a sub-partition of $X$ as blocks.

Let $P, Q \in \operatorname{Subpart}(X)$. By $P \leq Q$, we mean $P$ refines $Q$, i.e. for all $B \in P$, there exists $C \in Q$ such that $B \subseteq C$. For example, let $X=\left\{x_{1}, x_{2}, x_{3}\right\}$ and consider the sub-partitions $P:=\left\{\left\{x_{1}\right\},\left\{x_{2}\right\}\right\}$ and $Q:=\left\{\left\{x_{1}, x_{2}\right\},\left\{x_{3}\right\}\right\}$ of $X$. Then, it is easy to see that $P \leq Q$. 


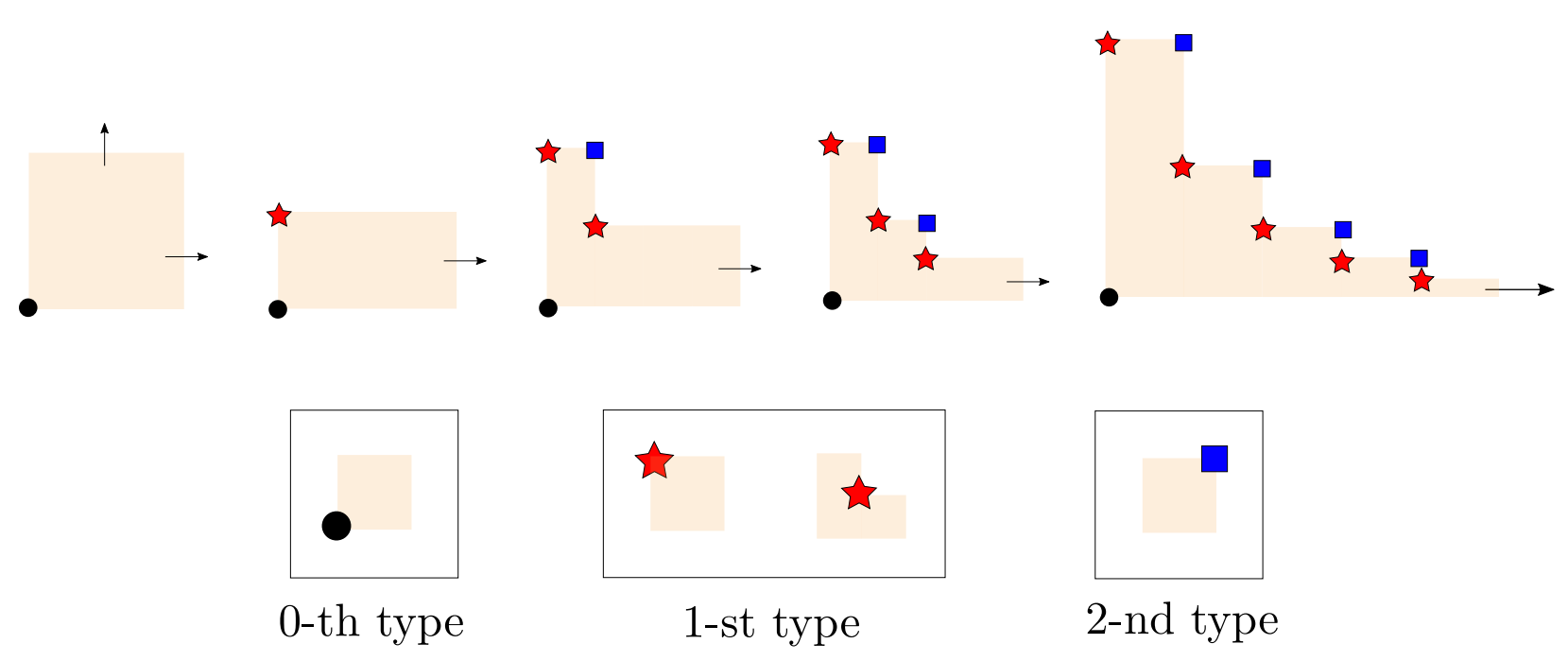

Figure 4: Every corner point of a staircase interval falls into three different types depending on its neighborhood information, as the pictures above illustrate. Staircase intervals in the first row are decorated by their corner points (a precise description is in Definition A.1 of Appendix).

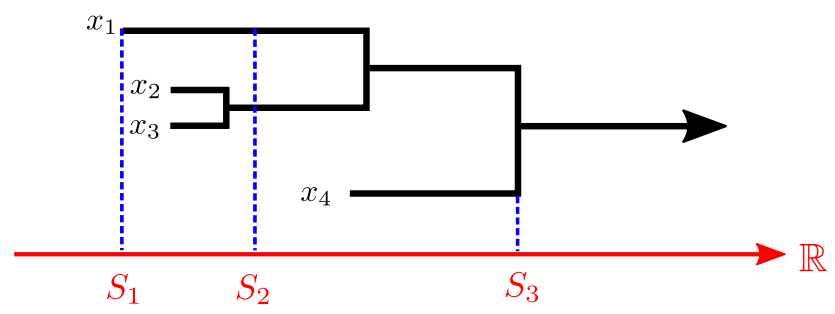

Figure 5: A (1D) treegram $\theta_{X}$ over the set $X:=\left\{x_{1}, x_{2}, x_{3}, x_{4}\right\}$. Notice that $\theta_{X}(t)=\emptyset$ for $t \in\left(-\infty, S_{1}\right)$. Also, $\theta_{X}\left(S_{1}\right)=\left\{\left\{x_{1}\right\}\right\}, \theta_{X}\left(S_{2}\right)=\left\{\left\{x_{1}\right\},\left\{x_{2}, x_{3}\right\}\right\}$, and $\theta_{X}(t)=\{X\}$ for all $t \in\left[S_{3}, \infty\right)$.

Treegrams are a generalized notion of dendrograms [41], which are useful for visualizing the evolution of clustering information of 1-parameter simplicial filtrations:

Definition 4.1 (Treegrams [41]). A treegram over a finite set $X$ is any function $\theta_{X}: \mathbb{R} \rightarrow$ $\operatorname{Subpart}(X)$ such that the following properties hold: (1) if $t_{1} \leq t_{2}$, then $\theta_{X}\left(t_{1}\right) \leq \theta_{X}\left(t_{2}\right)$, (2) there exists $T>0$ such that $\theta_{X}(t)=\{X\}$ for $t \geq T$ and $\theta_{X}(t)$ is empty for $t \leq-T$, and (3) for all $t$ there exists $\epsilon>0$ s.t. $\theta_{X}(s)=\theta_{X}(t)$ for $s \in[t, t+\epsilon]$. See Figure 5 for an example. Also, even when the domain $\mathbb{R}$ is replaced by any totally ordered set $L$ isomorphic to $\mathbb{R}, \theta_{X}$ is said to be a (1-parameter) treegram.

Given a simplicial complex $K$, let $K^{(0)}$ be the vertex set of $K$. Let $\pi_{0}(K)$ be the partition of the vertex set $K^{(0)}$ according to the connected components of $K$. A functor $\mathcal{K}: \mathbb{P} \rightarrow$ Simp is said to be a filtration of $K$ if $\mathcal{K}(\mathbf{a}) \subseteq K$ for all $\mathbf{a} \in \mathbb{P}$, every internal map is an inclusion, and there exists $\mathbf{a}_{0} \in \mathbb{P}$ such that for all $\mathbf{a} \in \mathbb{P}$ with $\mathbf{a}_{0} \leq \mathbf{a}, \mathcal{K}(\mathbf{a})=K$. 


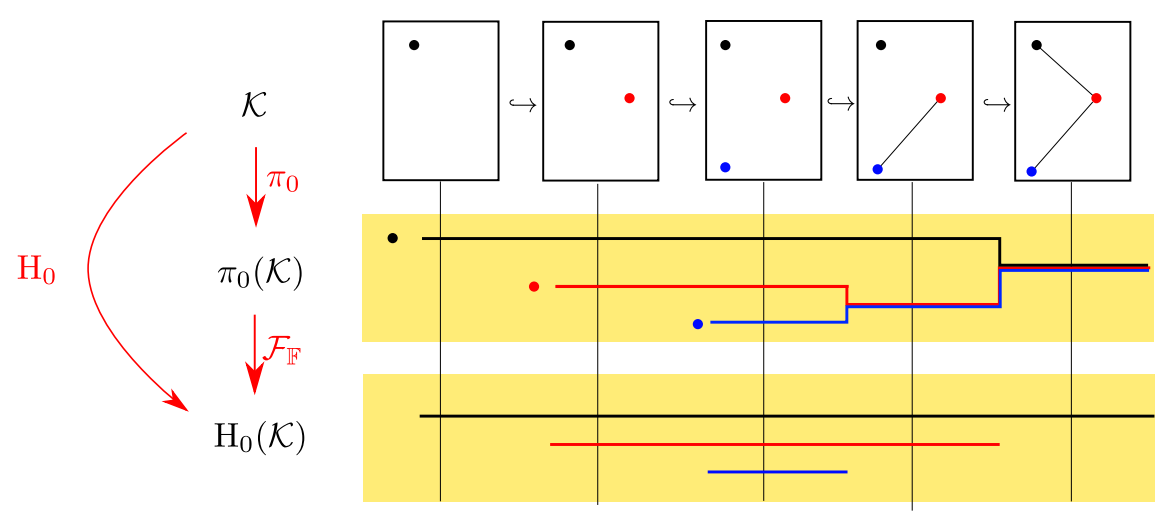

Figure 6: The first row represents a simplicial filtration $\mathcal{K}$. The second row stands for the the treegram $\pi_{0}(\mathcal{K})$ which encodes the evolution of clusters in $\mathcal{K}$ (Remark 4.2). The third row is the barcode of $\mathrm{H}_{0}(\mathcal{K})$. The persistence module $\mathrm{H}_{0}(\mathcal{K})$ can be obtained by applying the linearization functor (Definition 4.14 in Appendix) to $\pi_{0}(\mathcal{K})$. Alternatively, the barcode of $\mathrm{H}_{0}(\mathcal{K})$ can also be obtained by applying the elder rule to $\pi_{0}(\mathcal{K})$ (Definition 4.3).

Remark 4.2 (Treegrams induced by simplicial filtrations). Let $K$ be a simplicial complex on the vertex set $X=\left\{x_{1}, x_{2}, \ldots, x_{n}\right\}$ and let $\mathcal{K}: \mathbb{R} \rightarrow \operatorname{Simp}$ be a filtration of $K$. Assume that $K$ consists solely of one connected component, i.e. $\pi_{0}(K)=\{X\}$. Then, the function $\pi_{0}(\mathcal{K}): \mathbb{R} \rightarrow \operatorname{Subpart}(X)$ defined as $\varepsilon \mapsto \pi_{0}(\mathcal{K}(\varepsilon))$ is a treegram over $X$.

The zeroth elder rule for a 1-parameter filtration. Let $\theta_{X}$ be a treegram over $X$. We define the birth time of $x$ as $b(x):=\min \left\{\varepsilon \in \mathbb{R}: x\right.$ is in the underlying set of $\left.\theta_{X}(\varepsilon)\right\}$ (by Definition 4.1 (2) and (3), every $x \in X$ has the birth time $b(x)$ ). Pick any order $<$ on $X$ such that $b(x)<b\left(x^{\prime}\right)$ implies $x<x^{\prime}$ for all $x, x^{\prime} \in X .{ }^{1}$ For $\varepsilon \in[b(x), \infty)$, we denote the block to which $x$ belong in the sub-partition $\theta_{X}(\varepsilon)$ by $[x]_{\varepsilon}$. We define the death time of $x$ as $d^{<}(x)=\sup \left\{\varepsilon \in[b(x), \infty]: x=\min \left([x]_{\varepsilon},<\right)\right\}$. As long as $<$ is compatible with the birth times, the elder-rule-barcode is uniquely defined (which will be proved in Appendix):

Definition 4.3 (Elder-rule-barcode of a treegram). Let $\theta_{X}: \mathbb{R} \rightarrow \operatorname{Subpart}(X)$ be a treegram over $X$. For any order $<$ on $X$ compatible with the birth times, let $J_{x}:=\left[b(x), d^{<}(x)\right)$. The elder-rule-barcode of $\theta_{X}$ is defined as the multiset $\operatorname{barc}\left(\theta_{X}\right):=\left\{\left\{J_{x}: x \in X\right\}\right.$.

For the 1-parameter case, the elder-rule-barcode of a treegram can be obtained by dismantling the treegram into linear pieces w.r.t. the elder rule - see the theorem below. Even though this result is well-known (e.g, [21]), we include a proof at the end of this section.

Theorem 4.4 (Compatibility between the elder rule and algebraic decomposition). Let $\mathcal{K}$ and $\theta_{X}$ be the filtration and the treegram in Remark 4.2, respectively. Let $\operatorname{barc}\left(\theta_{X}\right)=$ $\left\{\left[J_{x}: x \in X\right\}\right.$ be the elder-rule-barcode of $\theta_{X}$. Then, $\mathrm{H}_{0}(\mathcal{K}) \cong \bigoplus_{x \in X} \mathcal{I}^{J_{x}}$ (see Figure 6 ).

We are now ready to prove Theorem 3.7.

\footnotetext{
${ }^{1}$ This order $<$ is uniquely specified if all $x \in X$ have different birth times.
} 
Proof of Theorem 3.7. Fix $L \in \mathcal{L}$. Since $L$ is isomorphic to $\mathbb{R}$ as a totally ordered set, $\mathcal{K}=\left.\mathcal{R}_{\bullet}^{\text {bi }}(\mathcal{X})\right|_{L}: L \rightarrow$ Simp can be viewed as a 1-parameter filtration. Consider the treegram $\theta_{X}:=\pi_{0}(\mathcal{K}): L \rightarrow \operatorname{Subpart}(X)$. By the definition of $I_{x} \mathrm{~s}$, it is clear that $\left\{L \cap I_{x}: x \in X\right\}$ is the elder-rule-barcode of the treegram $\theta_{X}$ (Definition 4.3). Hence, by Theorem 4.4, the multiset $\left\{\left\{\cap \cap I_{x}: x \in X\right\}\right.$ is equal to the barcode of $\mathrm{H}_{0}(\mathcal{K})$. Since $\mathrm{H}_{0}(\mathcal{K})=\left.M\right|_{L}$, we have $\left\{L \cap I_{x}: x \in X\right\}=\operatorname{barc}\left(\left.M\right|_{L}\right)$.

Bipersistence treegrams. We now extend the notion of treegrams to encode the evolution of clusters of a 2-parameter filtration (similar ideas appear in [29]). A bipersistence treegram over a finite set $X$ is any function $\theta_{X}^{\text {bi }}: \mathbb{R}^{2} \rightarrow \operatorname{Subpart}(X)$ such that if $\mathbf{a} \leq \mathbf{b}$ in $\mathbb{R}^{2}$, then $\theta_{X}^{\mathrm{bi}}(\mathbf{a}) \leq \theta_{X}^{\mathrm{bi}}(\mathbf{b})$.

We induce a bipersistence treegram over $X$ from an aug-MS $\mathcal{X}$.

Definition 4.5 (Rips bipersistence treegrams). Let $\mathcal{X}=\left(X, d_{X}, f_{X}\right)$ be an aug-MS. We define $\theta_{\mathcal{X}}^{\text {bi }}: \mathbb{R}^{2} \rightarrow \operatorname{Subpart}(X)$ as $(\sigma, \varepsilon) \mapsto \pi_{0}\left(\mathcal{R}_{\varepsilon}\left(X_{\sigma}, d_{X}\right)\right)$. This $\theta_{\mathcal{X}}^{\text {bi }}$ is said to be the Rips bipersistence treegram of $\mathcal{X}$.

Observe that $x \in X$ belongs to the underlying set of $\theta_{\mathcal{X}}^{\text {bi }}(\mathbf{a})$ if and only if $\left(f_{X}(x), 0\right) \leq \mathbf{a}$, i.e. $\left(f_{X}(x), 0\right)$ is the birth grade of $x$ in $\theta_{\mathcal{X}}^{\text {bi }}$. Assume that $\mathcal{X}$ is injective. Then the birth grades of elements in $X$ is totally ordered. Note that the ER-staircode of $\mathcal{X}$ can be extracted from $\theta_{\mathcal{X}}^{\text {bi }}$ Indeed, $I_{x}$ in equation (2) can be rephrased as $I_{x}=\left\{(\sigma, \varepsilon) \in \mathbb{R}^{2}: x\right.$ is in the underlying set of $\theta_{\mathcal{X}}^{\text {bi }}(\sigma, \varepsilon)$ and $x$ has the smallest birth grade in its block of $\left.\theta_{\mathcal{X}}^{\text {bi }}(\sigma, \varepsilon)\right\}$. See Figure 7 .

Definition 4.6 (Fibered treegrams). Let $\theta_{\mathcal{X}}^{\text {bi }}$ be a Rips bipersistence treegram of an aug-MS $\mathcal{X}$. The fibered treegram of $\theta_{\mathcal{X}}^{\text {bi }}$ refers to the collection $\left\{\left.\theta_{\mathcal{X}}^{\text {bi }}\right|_{L}\right\}_{L \in \mathcal{L}}$ of treegrams obtained by restricting $\theta_{\mathcal{X}}^{\text {bi }}$ to positive-slope lines (see Figure 8 for an example).

A combinatorial analogue of Theorem 2.5. Recall the elder-rule feature functions of an aug-MS $\mathcal{X}$ (Definition 3.9). We will show that they can be used to retrieve the cardinality function of $\theta_{\mathcal{X}}^{\text {bi }}$.

Definition 4.7 (Cardinality function). Let $\theta_{X}^{\text {bi }}$ be a bipersistence treegram over a set $X$. We call the function $\left|\theta_{X}^{\mathrm{bi}}\right|: \mathbb{R}^{2} \rightarrow \mathbb{Z}_{\geq 0}$ defined as $\mathbf{a} \mapsto\left|\theta_{X}^{\text {bi }}(\mathbf{a})\right|$, the cardinality function of $\theta_{X}^{\text {bi }}$.

For $A \subseteq \mathbb{R}^{2}$ we define the indicator function $\mathbb{1}_{A}: \mathbb{R}^{2} \rightarrow \mathbb{Z}_{\geq 0}$ of $A$ as

$$
\mathbb{1}_{A}(\mathbf{a}):= \begin{cases}1, & \mathbf{a} \in A, \\ 0, & \text { otherwise }\end{cases}
$$

The following proposition directly follows [22, Proposition 32]:

Proposition 4.8. Let $I$ be a staircase interval. Then, $\mathbb{1}_{I}(\mathbf{a})=\sum_{\mathbf{x} \leq \mathbf{a}} \sum_{j=0}^{2}(-1)^{j} \gamma_{j}(I)(\mathbf{x})$.

The ER-staircode and elder-rule feature functions of an aug-MS $\mathcal{X}$ recovers the cardinality function of $\theta_{\mathcal{X}}^{\text {bi }}$, which is analogous to Theorem 2.5: 

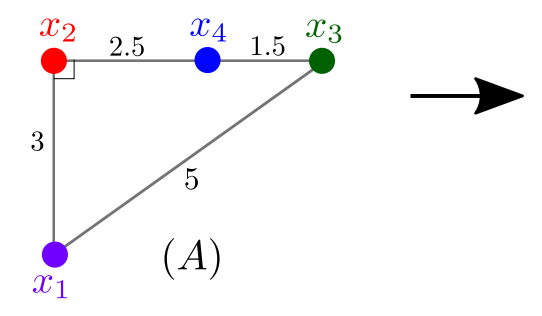

$(B)$
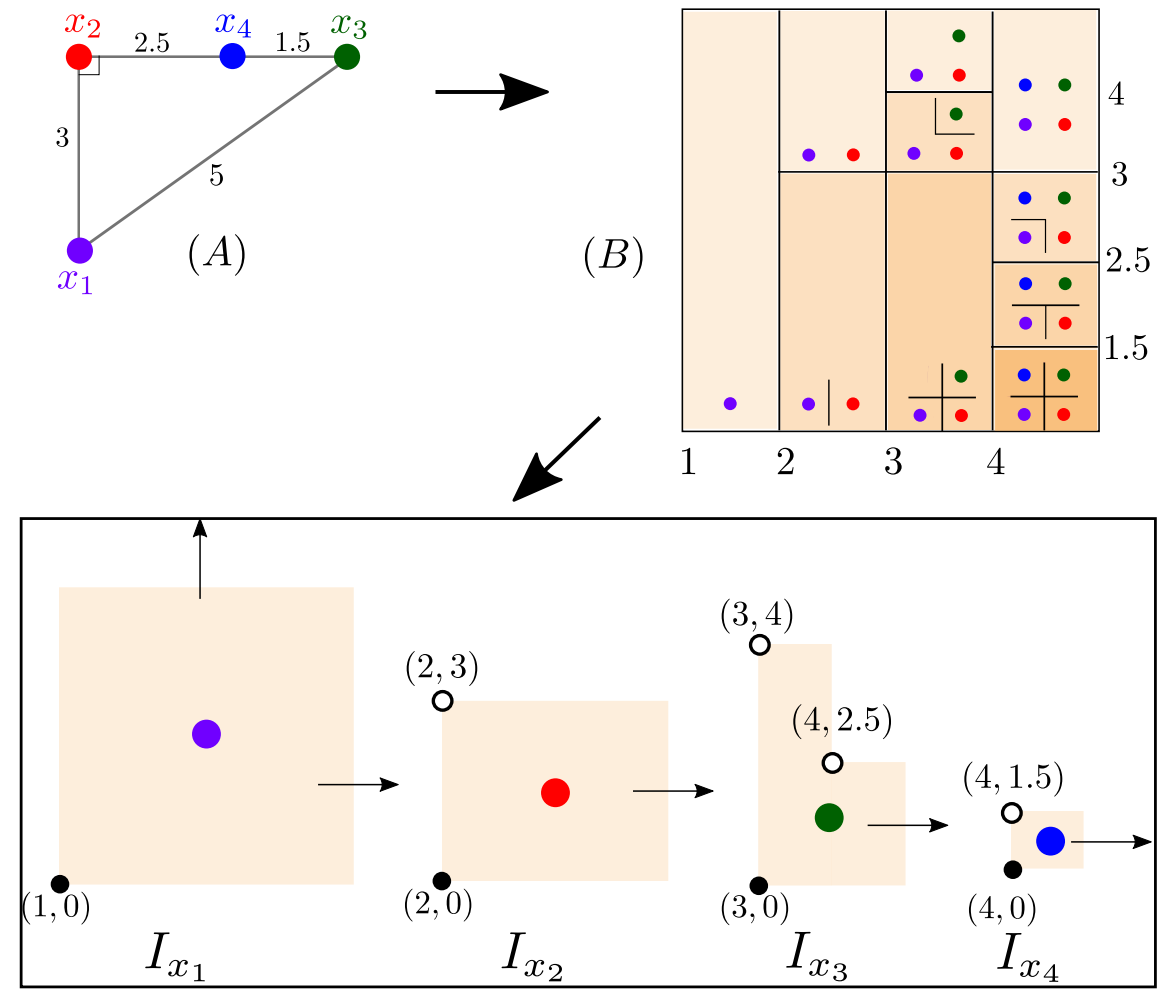

(C)

Figure 7: Consider the aug-MS $\mathcal{X}$ defined in Figure 1. Figure (A) and (C) above are identical to Figure $1(\mathrm{~A})$ and (B), respectively. (B) The Rips bipersistence treegram of $\mathcal{X}$ (Definition 4.5). The summarization processes $(\mathrm{A}) \rightarrow(\mathrm{B}) \rightarrow(\mathrm{C})$ are analogous to the processes depicted in Figure 6. Figures are best viewed in color.

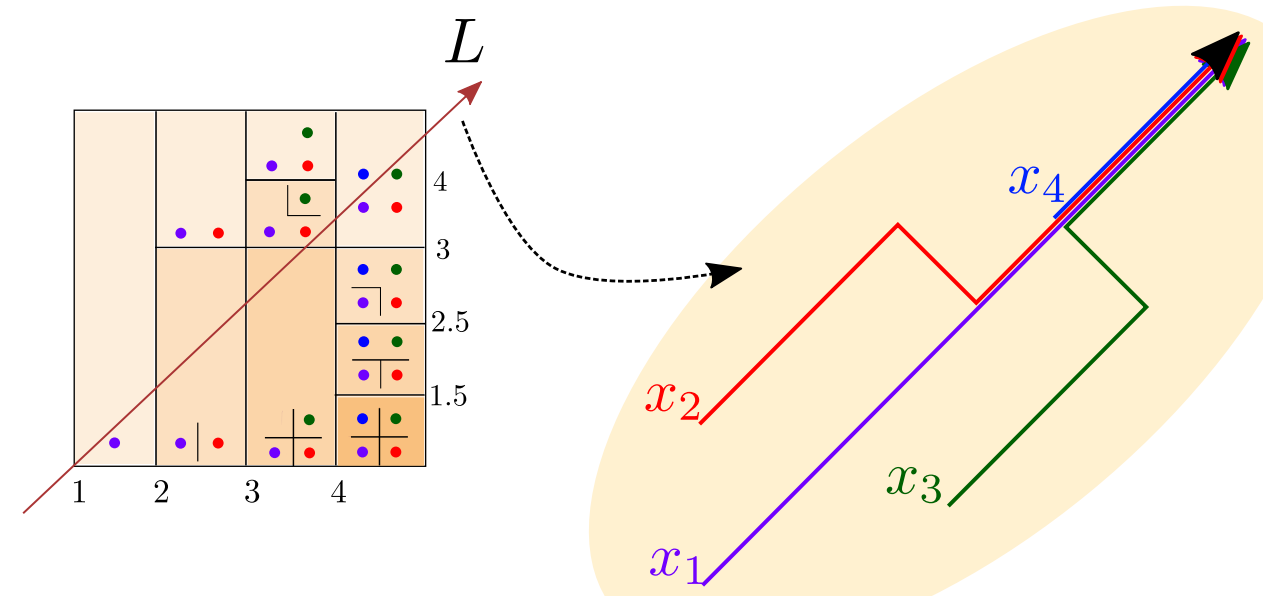

Figure 8: Consider the bipersistence treegram in Figure 7 (B) and pick a line $L$ of positive slope. Then, we obtain a treegram over $L$. 
Theorem 4.9. Let $\mathcal{X}$ be an aug-MS and let $I_{\mathcal{X}}=\left\{\left\{I_{x}: x \in X\right\}\right.$ be an ER-staircode of $\mathcal{X}$. For each $\mathbf{a} \in \mathbb{R}^{2}$,

$$
\begin{aligned}
\left|\theta_{\mathcal{X}}^{\mathrm{bi}}(\mathbf{a})\right| & =\left(\text { The number of intervals } I_{x} \in \mathcal{I}_{\mathcal{X}} \text { containing } \mathbf{a}\right) . \\
& =\sum_{\mathbf{x} \leq \mathbf{a}} \sum_{j=0}^{2}(-1)^{j} \gamma_{j}^{\mathcal{X}}(\mathbf{x}) .
\end{aligned}
$$

Proof. For simplicity we assume the injectivity of $\mathcal{X}$. We prove the equality in (5). Let $(\sigma, \varepsilon) \in \mathbb{R}^{2}$. Since each block in $\theta_{\mathcal{X}}^{\text {bi }}(\sigma, \varepsilon)$ contains its unique oldest element, $\left|\theta_{\mathcal{X}}^{\text {bi }}(\sigma, \varepsilon)\right|$ is equal to the cardinality of the set

$$
A(\sigma, \varepsilon):=\left\{x \in X_{\sigma}: x \text { is the oldest in the block containing } x \text { in } \theta_{X}(\sigma, \varepsilon)\right\} .
$$

By equation (2), a belongs to $I_{x_{i}}$ if and only if $x_{i} \in A(\sigma, \varepsilon)$, implying the equality

$$
|A(\sigma, \varepsilon)|=\left(\text { The number of intervals } I_{x_{i}} \in \mathcal{I}_{\mathcal{X}} \text { containing }(\sigma, \varepsilon)\right),
$$

as desired. The equality in (6) directly follows from Proposition 4.8 and Definition 3.9.

\subsection{Elder-rule-staircodes and fibered treegrams}

In this section we identify a sufficient condition on an aug-MS $\mathcal{X}$ for its ER-staircode to coincide with the barcode of the 2-parameter persistence module $\mathrm{H}_{0}\left(\mathcal{R}_{\bullet}^{\mathrm{bi}}(\mathcal{X})\right.$ ) (Theorem 4.10). Also, in general, all fibered treegrams can be recovered from ER-staircodes (Theorem 4.13).

Let $\left(X, d_{X}\right)$ be a metric space and fix $x, x^{\prime} \in X$. Recall that an $\varepsilon$-chain between $x$ and $x^{\prime}$ is a finite sequence $x=x_{1}, x_{2}, \ldots, x_{\ell}=x^{\prime}$ in $X$ where each consecutive pair $x_{i}, x_{i+1}$ is within distance $\varepsilon$. Define (in fact an ultrametric) $u_{X}: X \times X \rightarrow \mathbb{R}_{\geq 0}$ as

$$
u_{X}\left(x, x^{\prime}\right):=\min \left\{\varepsilon \in[0, \infty): \text { there exists an } \varepsilon \text {-chain between } x \text { and } x^{\prime}\right\} \text { (see [10]). }
$$

For a metric space $\left(X, d_{X}\right)$ and pick any total order $<$ on $X$. Let $x \in X$ be a non-minimal element of $(X,<)$. A $<$-conqueror of $x$ is an element $x^{\prime} \in X$ such that $(1) x^{\prime}<x$, and (2) for any $x^{\prime \prime} \in X$ with $x^{\prime \prime}<x$, it holds that $u_{X}\left(x, x^{\prime}\right) \leq u_{X}\left(x, x^{\prime \prime}\right)$.

Now consider an aug-MS $\mathcal{X}=\left(X, d_{X}, f_{X}\right)$. A <-conqueror function $c_{x}:\left[f_{X}(x), \infty\right) \rightarrow X$ of a non-minimal $x \in X$ sends $\sigma \in\left[f_{X}(x), \infty\right)$ to a conqueror of $x$ in $\left(X_{\sigma}, d_{X}\right)$. For the minimum $x^{\prime} \in(X,<)$, define $c_{x^{\prime}}:\left[f_{X}\left(x^{\prime}\right), \infty\right) \rightarrow X$ to be the constant function at $x^{\prime}$.

We generalize Theorem 4.4 and at the same time strengthen Theorem 3.7 for 2-parameter persistence modules induced by a special type of aug-MSs:

Theorem 4.10 (Compatibility between the ER-staircodes and algebraic decomposition). Let $\mathcal{X}=\left(X, d_{X}, f_{X}\right)$ be an aug-MS and fix any order $<$ on $X$ compatible with $f_{X}$. Assume that there exists a constant $<$-conqueror function for each $x \in X{ }^{2}$ Then, $\mathrm{H}_{0}\left(\mathcal{R}_{\bullet}^{\mathrm{bi}}(\mathcal{X})\right)$ is interval decomposable and its barcode coincides with the ER-staircode $\mathcal{I}_{\mathcal{X}}^{<}$.

\footnotetext{
${ }^{2}$ Observe that if this property holds for the order $<$, then the same property holds for any other order $<^{\prime}$ that is compatible with $f_{X}$, and $\mathcal{I}_{\mathcal{X}}^{<}=\mathcal{I}_{\mathcal{X}}^{<^{\prime}}$.
} 
The proof of Theorem 4.10 is similar to that of Theorem 4.4. The both proofs are given at the end of this section. Consider the aug-MS $\mathcal{X}$ in Figure 1. Observe that $\mathcal{X}$ satisfies the assumption in Theorem 4.10. Therefore, $\mathrm{H}_{0}\left(\mathcal{R}_{\bullet}^{\mathrm{bi}}(\mathcal{X})\right)$ is interval decomposable. There exists a class of aug-MSs to which Theorem 4.10 applies as shown by the following corollary.

Corollary 4.11. Let $\mathcal{X}=\left(X, d_{X}, f_{X}\right)$ be any aug-MS where $d_{X}$ is an ultrametric, i.e. $d_{X}\left(x, x^{\prime \prime}\right) \leq \max \left(d_{X}\left(x, x^{\prime}\right), d_{X}\left(x^{\prime}, x^{\prime \prime}\right)\right)$ for all $x, x^{\prime}, x^{\prime \prime} \in X$. Then, $\mathrm{H}_{0}\left(\mathcal{R}_{\bullet}^{\mathrm{bi}}(\mathcal{X})\right)$ is interval decomposable (in fact, its barcode consists solely of infinite rectangular intervals).

Proof. Let $<$ be an order on $X$ which is compatible with $f_{X}$. For each non-minimal $x \in$ $(X,<)$, pick an $x^{\prime} \in X$ such that (1) $x^{\prime}<x$, and (2) for any $x^{\prime \prime} \in X$ with $x^{\prime \prime}<x$, it holds that $d_{X}\left(x, x^{\prime}\right) \leq d_{X}\left(x, x^{\prime \prime}\right)$. Now observe that $x^{\prime}$ is a $<$-conqueror in $\left(X_{\sigma}, d_{X}\right)$ for every $\sigma \in\left[f_{X}(x), \infty\right)$, completing the proof.

The converse of Theorem 4.10 is false by virtue of the following example.

Example 4.12. Let $X:=\left\{x_{i}\right\}_{i=1}^{8}$. Consider $\mathcal{X}=\left(X, d_{X}, f_{X}\right)$ where $\left(X, d_{X}\right)$ is depicted in Figure 9 and $f_{X}\left(x_{i}\right)=i$ for each $i=1, \ldots, 8$. Then, $\mathrm{H}_{0}\left(\mathcal{R}^{\mathrm{bi}}(\mathcal{X})\right)$ is interval decomposable even though $x_{6} \in X$ does not have a constant conqueror. See below for the proofs of these claims.

Details from Example 4.12. The fact that $x_{6}$ does not have a constant conqueror can be ascertained from the following observation: For $\sigma \in[6,7), x_{1}, x_{2}$ and $x_{3}$ are the conquerors of $x_{6}$ in $X_{\sigma}$. For $\sigma \in[7,8), x_{3}, x_{4}$ and $x_{5}$ are the conquerors of $x_{6}$ in $X_{\sigma}$. For $\sigma \in[8, \infty), x_{5}$ is the unique conqueror of $x_{6}$ in $X_{\sigma}$.

Let $\mathcal{I}_{\mathcal{X}}=\left\{I_{x_{i}}\right\}_{i=1}^{8}$ be the ER-staircode of $\mathcal{X}$. To prove that $M:=\mathrm{H}_{0}\left(\mathcal{R}^{\mathrm{bi}}(\mathcal{X})\right)$ is interval decomposable, it suffices to construct an isomorphism $f$ from $N:=\bigoplus_{i=1}^{8} I^{I_{x_{i}}}$ to $M$. For $i=1, \ldots, 8$ and for $(\sigma, \varepsilon) \in[i, \infty) \times \mathbb{R}_{+}$, let $\left[x_{i}\right]_{(\sigma, \varepsilon)}$ be the zeroth homology class of $x_{i}$. When confusion is unlikely, we will suppress the subscript $(\sigma, \varepsilon)$ in $\left[x_{i}\right]_{(\sigma, \varepsilon)}$.

For each $i$, consider $1_{i}:=1 \in\left(I^{I_{x_{i}}}\right)_{(i, 0)}(=\mathbb{F})$. We declare that

$$
\begin{array}{ll}
1_{1} \stackrel{f_{(1,0)}}{\longmapsto}\left[x_{1}\right] & 1_{2} \stackrel{f_{(2,0)}}{\longmapsto}\left[x_{2}\right]-\left[x_{1}\right] \\
1_{3} \stackrel{f_{(3,0)}}{\longmapsto}\left[x_{3}\right]-\left[x_{1}\right] & 1_{4} \stackrel{f_{(4,0)}}{\longmapsto}\left[x_{4}\right]-\left[x_{3}\right] \\
1_{5} \stackrel{f_{(5,0)}}{\longmapsto}\left[x_{5}\right]-\left[x_{4}\right] & 1_{6} \stackrel{f_{((6,0)}}{\longmapsto}\left[x_{2}\right]-\left[x_{1}\right]+\left[x_{4}\right]-\left[x_{3}\right]+\left[x_{6}\right]-\left[x_{5}\right] \\
1_{7} \stackrel{f_{(7,0)}}{\longmapsto}\left[x_{7}\right]-\left[x_{3}\right] & 1_{8} \stackrel{f_{(8,0)}}{\longmapsto}\left[x_{8}\right]-\left[x_{6}\right] .
\end{array}
$$

Since $\left\{1_{i}: i=1, \ldots, 8\right\}$ is a set of all generators of $N$, the above specification gives rise to a unique morphism $f: N \rightarrow M$. It is not hard to check that $f$ is actually an isomorphism.

We enrich the ER-staircode in order to query the fibered treegram: Let $\mathcal{X}=\left(X, d_{X}, f_{X}\right)$ be an aug-MS. Let $<$ be any order on $X$ which is compatible with $f_{X}$. For each $x$, we define $I_{x}^{*}$ as the pair $\left(I_{x}, c_{x}\right)$ of the set $I_{x}$ and the <-conqueror function $c_{x}$. The collection $\mathcal{I}_{\mathcal{X}}^{*}:=\left\{I_{x}^{*}\right\}_{x \in X}$ is said to be the decorated ER-staircode of $\mathcal{X}$. See Figure 10. The following result is easy to obtain with the help of decorations.

Theorem 4.13. Given any $L \in \mathcal{L}$, the fibered treegram $\left.\theta_{\mathcal{X}}^{\text {bi }}\right|_{L}$ can be recovered from the decorated ER-staircode $\mathcal{I}_{\mathcal{X}}^{*}$ of the aug-MS $\mathcal{X}=\left(X, d_{X}, f_{X}\right)$. 


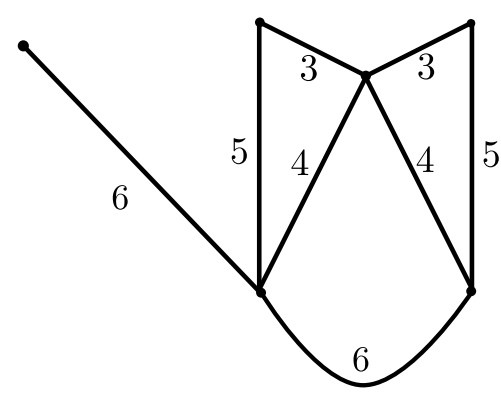

(A)

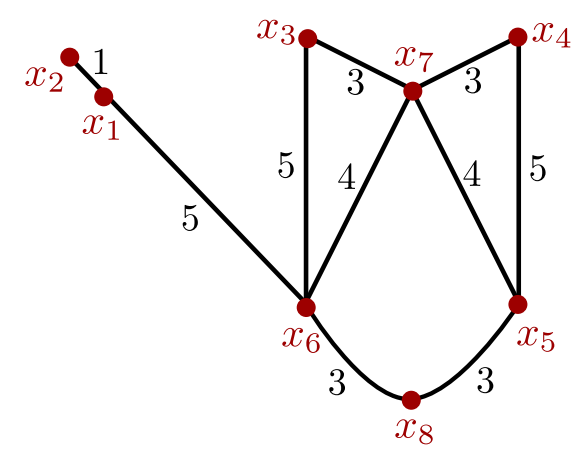

(B)

Figure 9: (A) A metric graph $G$. The distance between any two points on $G$ is the length of a shortest path connecting them. (B) The embedding of $\left(X, d_{X}\right)$ in $G$.

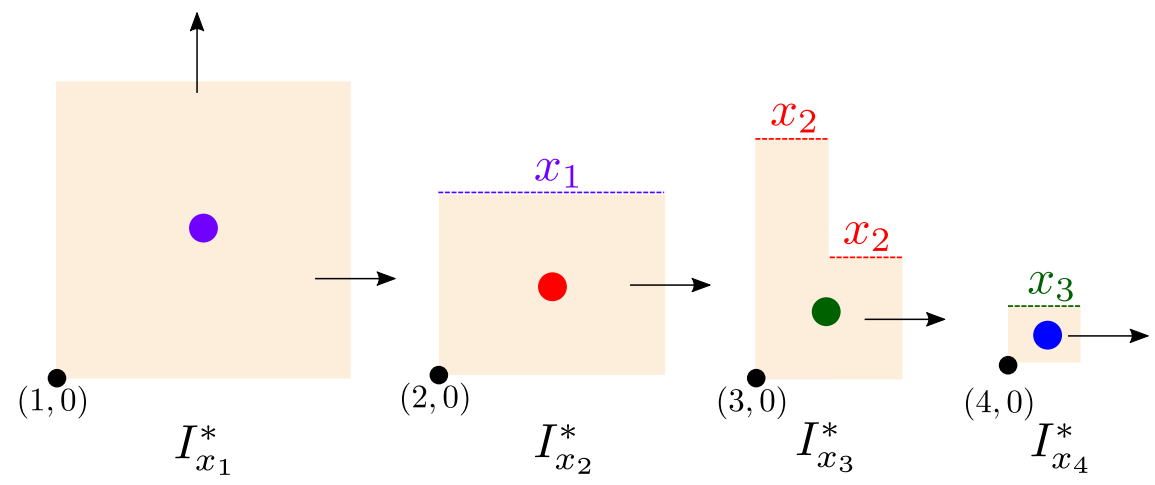

Figure 10: Decorated intervals corresponding to the four intervals in Figure 1 (C). For each $i=2,3,4$, the upper boundary of $I_{x_{i}}$ is decorated by the conqueror of $x_{i}$. 
Proofs of Theorems 4.4 and 4.10. We first define the linearization functor:

Definition 4.14 (Linearization functor). Let $X$ be a non-empty finite set. We define the linearization functor $\mathcal{F}_{\mathbb{F}}: \operatorname{Subpart}(X) \rightarrow$ Vec as follows.

(i) Each $P \in \operatorname{Subpart}(X)$ is sent to the vector space $\mathcal{F}_{\mathbb{F}}(B)$ which consists of formal linear combinations of elements of $P$ over the field $\mathbb{F}$. In other words,

$$
\mathcal{F}_{\mathbb{F}}(P)=\left\{\sum_{B \in P} c_{B} B: c_{B} \in \mathbb{F}\right\} .
$$

By identifying each $B \in P$ with $1 \cdot B \in \mathcal{F}_{\mathbb{F}}(P)$, the sub-partition $P$ can be viewed as a basis of $\mathcal{F}_{\mathbb{F}}(P)$.

(ii) Each pair $P \leq Q$ in $\operatorname{Subpart}(X)$ is sent to the linear map $\mathcal{F}_{\mathbb{F}}(P) \rightarrow \mathcal{F}_{\mathbb{F}}(Q)$ which sends each $1 \cdot B \in \mathcal{F}_{\mathbb{F}}(P)$ to $1 \cdot B^{\prime} \in \mathcal{F}_{\mathbb{F}}(Q)$ such that $B \subseteq B^{\prime}$.

The following proposition is straightforward by [38, Theorem 7.1]:

Proposition 4.15. $\quad$ (i) Let $\theta_{X}: \mathbb{R} \rightarrow \operatorname{Subpart}(X)$ be the treegram obtained by applying $\pi_{0}$ to a filtration $\mathcal{K}: \mathbb{R} \rightarrow \operatorname{Simp}$ (Remark 4.2). The two 1-parameter persistence modules $\mathcal{F}_{\mathbb{F}} \circ \theta_{X}$ and $\mathrm{H}_{0}(\mathcal{K})$ are isomorphic.

(ii) Let $\mathcal{X}$ be an aug-MS. The two 2-parameter persistence modules $\mathcal{F}_{\mathbb{F}} \circ \theta_{\mathcal{X}}^{\text {bi }}$ and $\mathrm{H}_{0}\left(\mathcal{R}_{\bullet}^{\text {bi }}(\mathcal{X})\right)$ (Definitions 3.2 and 4.5) are isomorphic.

Now we are ready to prove Theorems 4.4 and 4.10 .

Proof of Theorem 4.4. Without loss of generality, let $X=\left\{x_{1}, \ldots, x_{n}\right\}$. By Proposition 4.15 (i), $\mathrm{H}_{0}(\mathcal{K})$ is isomorphic to $M:=\mathcal{F}_{\mathbb{F}} \circ \theta_{X}$, and thus it suffices to show that $M \cong$ $\bigoplus_{i=1}^{n} I^{\left[b\left(x_{i}\right), d\left(x_{i}\right)\right)}=: N$. We may assume that $b\left(x_{1}\right) \leq b\left(x_{2}\right) \leq \ldots \leq b\left(x_{n}\right)$. For each $i \in$ $\{2,3, \ldots, n\}$, we pick a certain $x_{q(i)}$ which merges with $x_{i}$ earliest in the treegram $\theta_{X}$ among all the points in $\left\{x_{1}, x_{2}, \ldots, x_{i-1}\right\}$. This defines a function $q:\{2,3, \ldots, n\} \rightarrow\{1,2, \ldots, n\}$ (such function $q$ is not necessarily unique, since some two points $x_{j_{1}}, x_{j_{2}}$ might merge with another point $x_{j_{3}}$ at the same time).

For $x_{i} \in X$ and $\varepsilon \in\left[b\left(x_{i}\right), \infty\right)$, let $\left[x_{i}\right]_{\varepsilon}$ be the block containing $x_{i}$ in the sub-partition $\theta_{X}(\varepsilon)$ of $X$.

On the interval $\left(-\infty, b\left(x_{1}\right)\right)$, both $M$ and $N$ are trivial and thus let $f_{\varepsilon}$ be the zero map for $\varepsilon \in\left(-\infty, b\left(x_{1}\right)\right)$.

Fix $\varepsilon \in\left[b\left(x_{1}\right), \infty\right)$. Note that the vector space $M(\varepsilon)$ is spanned by $\mathcal{A}=\left\{\left[x_{i}\right]_{\varepsilon} \in \theta_{X}(\varepsilon)\right.$ : $\left.b\left(x_{i}\right) \leq \varepsilon\right\}$. Therefore, $M(\varepsilon)$ is also spanned by $\mathcal{B}=\left\{\left[x_{i}\right]_{\varepsilon}-\left[x_{q(i)}\right]_{\varepsilon}: b\left(x_{i}\right) \leq \varepsilon\right\}$, which is obtained by applying elementary linear operations on $\mathcal{A}$. Furthermore, observe that

$$
\mathcal{B}^{\prime}=\left\{\left[x_{1}\right]_{\varepsilon}\right\} \cup\left(\left\{\left[x_{i}\right]_{\varepsilon}-\left[x_{q(i)}\right]_{\varepsilon}: b\left(x_{i}\right) \leq \varepsilon\right\} \backslash\{0\}\right)
$$

is a linearly independent set and in turn a basis of $M(\varepsilon)$. Define the linear map $f_{\varepsilon}: M(\varepsilon) \rightarrow$ $N(\varepsilon)$ by defining it on the basis $\mathcal{B}^{\prime}$ as follows:

(i) send $\left[x_{1}\right]_{\varepsilon}$ to 1 in the 1 -st summand of $N(\varepsilon)=\bigoplus_{i=1}^{n} I^{\left[b\left(x_{i}\right), d\left(x_{i}\right)\right)}(\varepsilon)$. 
(ii) send each basis element $\left[x_{i}\right]_{\varepsilon}-\left[x_{q(i)}\right]_{\varepsilon}(\neq 0)$ to 1 in the $i$-th summand of

$$
\bigoplus_{i=1}^{n} I^{\left[b\left(x_{i}\right), d\left(x_{i}\right)\right)}(\varepsilon) .
$$

Then, one can check that the collection $f=\left\{f_{\varepsilon}\right\}_{\varepsilon \in \mathbb{R}}$ is an isomorphism between $M$ and $N$, as desired.

We make use of the same strategy as Theorem 4.4 for proving Theorem 4.10:

Proof of Theorem 4.10. Without loss of generality, we may assume that $X=\left\{x_{1}, \ldots, x_{n}\right\}$, $f_{X}\left(x_{1}\right) \leq f_{X}\left(x_{2}\right) \leq \ldots \leq f_{X}\left(x_{n}\right)$, and let the order $<$ on $X$ defined as $\left(x_{1}<x_{2}<\ldots<x_{n}\right)$. Also, assume that each <-conqueror function $c_{x_{i}}: \mathbb{R} \rightarrow X$ is constant at $q(i) \in X$ (then by definition $q(1)=x_{1}$ ). By Proposition 4.15 (ii), it suffices to show that $M:=\mathcal{F}_{\mathbb{F}} \circ \theta_{\mathcal{X}}^{\text {bi }}$ is isomorphic to $N=\bigoplus_{i=1}^{n} I^{I_{x_{i}}^{<}}$.

For $x_{i} \in X$, and $(\sigma, \varepsilon) \in \mathbb{R}^{2}$ with $(\sigma, \varepsilon) \geq\left(f\left(x_{i}\right), 0\right)$, let $\left[x_{i}\right]_{(\sigma, \varepsilon)}$ be the block containing $x_{i}$ in the sub-partition $\theta_{\mathcal{X}}^{\text {bi }}(\sigma, \varepsilon)$ of $X$.

For any $(\sigma, \varepsilon) \in \mathbb{R}^{2}$ such that $(\sigma, \varepsilon) \nsupseteq\left(f_{X}\left(x_{1}\right), 0\right)$, both $M(\sigma, \varepsilon)$ and $N(\sigma, \varepsilon)$ are trivial and thus let $f_{(\sigma, \varepsilon)}$ be the zero map for $(\sigma, \varepsilon) \nsupseteq\left(f_{X}\left(x_{1}\right), 0\right)$.

Fix $(\sigma, \varepsilon) \in \mathbb{R}^{2}$ such that $(\sigma, \varepsilon) \geq\left(f_{X}\left(x_{1}\right), 0\right)$. The vector space $M(\sigma, \varepsilon)$ is spanned by $\mathcal{A}=\left\{\left[x_{i}\right]_{(\sigma, \varepsilon)} \in \theta_{\mathcal{X}}^{\text {bi }}(\sigma, \varepsilon):\left(f_{X}\left(x_{i}\right), 0\right) \leq(\sigma, \varepsilon)\right\}$. Therefore, $M(\sigma, \varepsilon)$ is also spanned by $\mathcal{B}=\left\{\left[x_{1}\right]_{(\sigma, \varepsilon)}\right\} \cup\left\{\left[x_{i}\right]_{(\sigma, \varepsilon)}-\left[x_{q(i)}\right]_{(\sigma, \varepsilon)}:\left(f_{X}\left(x_{i}\right), 0\right) \leq(\sigma, \varepsilon)\right\}$, which is obtained by applying elementary linear operations on $\mathcal{A}$. Furthermore, note that

$$
\mathcal{B}^{\prime}:=\left\{\left[x_{1}\right]_{(\sigma, \varepsilon)}\right\} \cup\left(\left\{\left[x_{i}\right]_{(\sigma, \varepsilon)}-\left[x_{q(i)}\right]_{(\sigma, \varepsilon)}:\left(f_{X}\left(x_{i}\right), 0\right) \leq(\sigma, \varepsilon)\right\} \backslash\{0\}\right)
$$

is a linearly independent set and in turn a basis of $M(\sigma, \varepsilon)$. Let us define a linear map $f_{(\sigma, \varepsilon)}: M(\sigma, \varepsilon) \rightarrow N(\sigma, \varepsilon)$ by defining it on the basis $\mathcal{B}^{\prime}$ as follows:

(i) send $\left[x_{1}\right]_{(\sigma, \varepsilon)}$ to 1 in the 1 -st summand of $N(\sigma, \varepsilon)=\bigoplus_{i=1}^{n} I^{I_{x_{i}}}(\sigma, \varepsilon)$.

(ii) send each basis element $\left[x_{i}\right]_{(\sigma, \varepsilon)}-\left[x_{q(i)}\right]_{(\sigma, \varepsilon)}(\neq 0)$ to 1 in the $i$-th summand of $N(\sigma, \varepsilon)=$ $\bigoplus_{i=1}^{n} I^{I_{x_{i}}}(\sigma, \varepsilon)$.

By invoking the construction of the $<$-conqueror functions $c_{x_{i}}$ and the ER-staircode $\mathcal{I}_{\mathcal{X}}^{<}=$ $\left\{\left\{I_{x_{i}}^{<}: i=1, \ldots, n\right\}\right\}$, one can check that the collection $f=\left\{f_{(\sigma, \varepsilon)}\right\}_{(\sigma, \varepsilon) \in \mathbb{R}^{2}}$ is an isomorphism between $M$ and $N$, as desired.

\subsection{Elder-rule-staircodes are the only candidates for the barcodes}

The compatibility between the elder-rule and the algebraic decomposition theory (Theorem 4.10) will be enhanced to Theorem 4.16 below. For any $\left(\sigma_{0}, \varepsilon_{0}\right) \in \mathbb{R}^{2}$, let $U\left(\sigma_{0}, \varepsilon_{0}\right):=$ $\left\{(\sigma, \varepsilon) \in \mathbb{R}^{2}:\left(\sigma_{0}, \varepsilon_{0}\right) \leq(\sigma, \varepsilon)\right\}$, i.e. the closed quadrant whose lower-left corner point is $\left(\sigma_{0}, \varepsilon_{0}\right)$.

Theorem 4.16. Let $\mathcal{X}$ be an injective aug-MS such that $M:=\mathrm{H}_{0}\left(\mathcal{R}_{\bullet}^{\mathrm{bi}}(\mathcal{X})\right)$ is interval decomposable. Then, the barcode of $M$ coincides with the ER-staircode $\mathcal{I}_{\mathcal{X}}^{<}$of $\mathcal{X}$. 
The proof utilizes results in Section 5 and thus is deferred to that section.

Remark 4.17. By Theorem 4.16, testing the interval decomposability of $\mathrm{H}_{0}\left(\mathcal{R}^{\text {bi }}(\mathcal{X})\right)$ is equivalent to testing whether $\mathrm{H}_{0}\left(\mathcal{R}^{\mathrm{bi}}(\mathcal{X})\right) \cong N:=\bigoplus_{i=1}^{n} I^{I_{x_{i}}}$. In [6] there exists a deterministic algorithm for testing such an isomorphism.

Stratification of the collection of augmented metric spaces Let us consider the following collections of aug-MSs.

(i) Aug is defined as the collection of all finite aug-MSs.

The following are sub-collections of Aug.

(ii) Ult consists of all finite $\operatorname{aug}-\operatorname{MSs}\left(X, d_{X}, f_{X}\right)$ where $d_{X}$ is an ultrametric.

(iii) $\operatorname{Rep}(e, m)$ consists of all finite aug-MSs $\mathcal{X}$ such that the horizontal internal maps of $\mathrm{H}_{0}\left(\mathcal{R}_{\bullet}^{\mathrm{bi}}(\mathcal{X})\right)$ are injective.

(iv) Rec consists of all finite aug-MSs $\mathcal{X}$ such that $\mathrm{H}_{0}\left(\mathcal{R}_{\bullet}^{\mathrm{bi}}(\mathcal{X})\right)$ is rectangle decomposable, i.e. each indecomposable summand is $I^{[a, b) \times[c, d)}$ for some intervals $[a, b),[c, d)$ of $\mathbb{R}$.

(v) ER consists of all finite aug-MSs $\mathcal{X}$ such that the assumption of Theorem 4.10 holds (and thus interval decomposable).

(vi) Dec consists of all finite aug-MSs $\mathcal{X}$ such that $\mathrm{H}_{0}\left(\mathcal{R}_{\bullet}^{\mathrm{bi}}(\mathcal{X})\right)$ is interval decomposable.

In order to clarify the relationship among these collections, we begin by recalling:

Theorem 4.18 ([4, Corollary 3.17]). $\boldsymbol{R e p}(e, m)=\mathbf{R e c}$.

We enrich Theorem 4.18 as follows:

Theorem 4.19. Ult $\subsetneq \operatorname{Rep}(e, m)=\operatorname{Rec} \subsetneq \mathrm{ER} \subsetneq \operatorname{Dec} \subsetneq$ Aug.

We in particular remark that Example 5.5 provides an aug-MS which does not belong to Dec. Such examples provide clues for how to construct aug-MSs $\mathcal{X}$ which yield $\mathrm{H}_{0}\left(\mathcal{R}^{\mathrm{bi}}(\mathcal{X})\right)$ whose isomorphism type is exotic, thus complementing the results of [4].

Proof. (i) Ult $\subseteq \boldsymbol{R e p}(e, m)$ : Consider an aug-MS $\mathcal{X}=\left(X, d_{X}, f_{X}\right)$ where $d_{X}$ is an ultrametric. By Proposition 4.15 (ii), it suffices to show that every horizontal internal map of $\theta_{X}^{\text {bi }}: \mathbb{R}^{2} \rightarrow \operatorname{Subpart}(X)$ is injective. Pick $\left(\sigma_{1}, \varepsilon\right),\left(\sigma_{2}, \varepsilon\right) \in \mathbb{R}^{2}$ with $\sigma_{1} \leq \sigma_{2}$ and pick $x, y \in X$ with $f_{X}(x), f_{X}(y) \leq \sigma_{1}$. Assume that $[x]_{\left(\sigma_{2}, \varepsilon\right)}=[y]_{\left(\sigma_{2}, \varepsilon\right)}$ and let us show that $[x]_{\left(\sigma_{1}, \varepsilon\right)}=[y]_{\left(\sigma_{1}, \varepsilon\right)}$. The assumption implies that there exists a sequence $x=x_{0}, \ldots, x_{n}=y$ in $X_{\sigma_{2}}$ such that $d_{X}\left(x_{i}, x_{i+1}\right) \leq \varepsilon$ for each $i$. Since $d_{X}$ is an ultrametric, we have that $d_{X}(x, y) \leq \max _{i=0}^{n-1} d_{X}\left(x_{i}, x_{i+1}\right) \leq \varepsilon$. Invoking $f_{X}(x), f_{X}(y) \leq \sigma_{1}$, we have $[x]_{\left(\sigma_{1}, \varepsilon\right)}=[y]_{\left(\sigma_{1}, \varepsilon\right)}$, as desired.

(ii) Ult $\neq \boldsymbol{\operatorname { R e p }}(e, m)$ : Let us equip the set $X:=\{1,2,3\}$ with the standard metric $d(i, j):=|i-j|, i, j \in\{1,2,3\}$, and the map $f: X \rightarrow \mathbb{R}$ defined as $i \mapsto i$ for $i=1,2,3$. Observe that $d_{X}$ is not an ultrametric, but every horizontal internal map of $\mathrm{H}_{0}\left(\mathcal{R}^{\mathrm{bi}}(\mathcal{X})\right)$ is injective. 
(iii) $\boldsymbol{R e p}(e, m) \subseteq \mathbf{E R}$ : Consider an aug-MS $\mathcal{X}=\left(X, d_{X}, f_{X}\right)$ in $\boldsymbol{R e p}(e, m)$. Pick an order $<$ on $X$ which is compatible with $f_{X}$. Let $x \in(X,<)$ be a non-minimal element and let $\sigma_{0}:=f_{X}(x)$. Let $x^{\prime}$ be a conqueror of $x$ in the metric space $\left(X_{\sigma_{0}}, d_{X}\right)$. It suffices to show that for each $\sigma \in\left[\sigma_{0}, \infty\right), x^{\prime}$ is a conqueror of $x$ in $\left(X_{\sigma}, d_{X}\right)$. Fix $\sigma \in\left[\sigma_{0}, \infty\right)$. Let $x^{\prime \prime} \in X_{\sigma}$ be a conqueror of $x$ in $\left(X_{\sigma}, d_{X}\right)$. Let $u_{X}^{\sigma}: X \times X \rightarrow \mathbb{R}$ be the (ultra)metric induced by $\left(X_{\sigma}, d_{X}\right)$ as in $(7)$. Let $\varepsilon:=u_{X}^{\sigma}\left(x^{\prime \prime}, x\right)$. By definition of $x^{\prime \prime}$, we have

$$
\varepsilon \leq u_{X}^{\sigma}\left(x^{\prime}, x\right) \text {. }
$$

Also, by definition of $\varepsilon$, we have $[x]_{(\sigma, \varepsilon)}=\left[x^{\prime \prime}\right]_{(\sigma, \varepsilon)}$. Since $\mathcal{X}$ belongs to $\operatorname{Rep}(e, m)$, it also holds that $[x]_{\left(\sigma_{0}, \varepsilon\right)}=\left[x^{\prime \prime}\right]_{\left(\sigma_{0}, \varepsilon\right)}$, implying

$$
u_{X}^{\sigma_{0}}\left(x, x^{\prime \prime}\right) \leq \varepsilon
$$

Since $x^{\prime}$ is a conqueror of $x$ in $\left(X_{\sigma_{0}}, d_{X}\right)$, we have:

$$
u_{X}^{\sigma_{0}}\left(x, x^{\prime}\right) \leq u_{X}^{\sigma_{0}}\left(x, x^{\prime \prime}\right) .
$$

Also, since $u_{X}^{\sigma} \leq u_{X}^{\sigma_{0}}$, we have:

$$
u_{X}^{\sigma}\left(x, x^{\prime}\right) \leq u_{X}^{\sigma_{0}}\left(x, x^{\prime}\right)
$$

By concatenating inequalities (8), (9), (10), and (11) we obtain:

$$
u_{X}^{\sigma}\left(x, x^{\prime}\right) \leq u_{X}^{\sigma_{0}}\left(x, x^{\prime \prime}\right) \leq \varepsilon \leq u_{X}^{\sigma}\left(x^{\prime}, x\right) \leq u_{X}^{\sigma}\left(x, x^{\prime}\right) .
$$

The both very end sides are the same, implying that $\varepsilon=u_{X}^{\sigma}\left(x, x^{\prime}\right)$. Since $\varepsilon=u_{X}^{\sigma}\left(x^{\prime \prime}, x\right)$ and $x^{\prime \prime}$ is a conqueror of $x$ in $\left(X_{\sigma}, d_{X}\right)$, we conclude that $x^{\prime}$ is another conqueror of $x$ in $\left(X_{\sigma}, d_{X}\right)$, as desired.

(iv) $\boldsymbol{R e p}(e, m) \neq \mathbf{E R}$ : It is not hard to check that the aug-MS depicted in Figure 1 (A) belongs to $\mathbf{E R}$ but not $\boldsymbol{\operatorname { R e p }}(e, m)$.

(v) ER $\subsetneq$ Dec: This follows from Theorem 4.10 and Example 5.5.

(vi) Dec $\subsetneq$ Aug: This directly follows from Example 5.5.

\section{$5 \quad$ Elder-rule-staircodes and graded Betti numbers}

In this section we show that given an aug-MS $\mathcal{X}$ the graded Betti numbers of $\mathrm{H}_{0}\left(\mathcal{R}_{\bullet}^{\mathrm{bi}}(\mathcal{X})\right)$ can be easily extracted from the ER-staircode of $\mathcal{X}$ (Theorem 5.4). Along the way, we obtain

a characterization result for the graded Betti number of $\mathrm{H}_{0}\left(\mathcal{R}_{\bullet}^{\mathrm{bi}}(\mathcal{X})\right.$ ) (Theorem 5.2), which is of independent interest. 
Computing the graded Betti numbers of $\mathrm{H}_{0}\left(\mathcal{R}_{\bullet}^{\mathrm{bi}}(\mathcal{X})\right)$ for an aug-MS $\mathcal{X}$. Henceforth, for simplicity, every aug-MS $\mathcal{X}=\left(X, d_{X}, f_{X}\right)$ is assumed to be generic: $f_{X}$ is injective and every pair of elements in $X$ has different distance. The case of non-generic aug-MS can be easily handled; see Remark 5.6. Since $\mathcal{X}$ is finite, it suffices to consider $\mathbb{Z}^{2}$-indexed filtration described subsequently as a substitute of $\mathcal{R}_{\bullet}^{\text {bi }}(\mathcal{X})$ for our inductive proof of Theorem 5.4:

Definition 5.1. Consider an aug- $M S \mathcal{X}=\left(X, d_{X}, f_{X}\right)$ with $X:=\left\{x_{1}, \ldots, x_{n}\right\}$ and assume that $f_{X}\left(x_{1}\right)<\ldots<f_{X}\left(x_{n}\right)$. Define $f_{X}^{\mathbb{Z}}: X \rightarrow \mathbb{N}$ as $x_{i} \mapsto i$. Define $d_{X}^{\mathbb{Z}}: X \times X \rightarrow \mathbb{N}$ by sending each non-trivial pair $\left(x_{i}, x_{j}\right)(i \neq j)$ to $\ell \in\left\{1, \ldots,\left(\begin{array}{l}n \\ 2\end{array}\right)\right\}$, where $d_{X}\left(x_{i}, x_{j}\right)$ is the $\ell$-th smallest distance (among non-zero distance values). The restriction of $\mathcal{R}_{\bullet}^{\mathrm{bi}}\left(X, d_{X}^{\mathbb{Z}}, f_{X}^{\mathbb{Z}}\right)$ : $\mathbb{R}^{2} \rightarrow$ Simp to $\mathbb{Z}^{2}$ is the $\mathbb{Z}^{2}$-indexed Rips filtration ${ }^{3}$ of $\mathcal{X}$. Also, let $\gamma_{j}^{\mathcal{X}}$ denote the $j$-th elder-rule feature function of $\left(X, d_{X}^{\mathbb{Z}}, f_{X}^{\mathbb{Z}}\right)$ for $j=0,1,2$ in this section.

For Theorem 5.2, we introduce relevant terminology and notation. Let $\mathcal{S}$ be the $\mathbb{Z}^{2}$ indexed Rips filtration of an aug-MS $\mathcal{X}$ and let $\mathcal{K}$ be the 1-skeleton of $\mathcal{S}$, i.e. $\mathcal{K}$ is another $\mathbb{Z}^{2}$-indexed filtration where $\mathcal{K}(\mathbf{a})$ is the 1 -skeleton of $\mathcal{S}(\mathbf{a})$ for every $\mathbf{a} \in \mathbb{P}$.

- Note that $\mathcal{K}$ is 1 -critical: every simplex that appears in $\mathcal{K}$ has a unique birth index. Let $e$ be an edge that appears in $\mathcal{K}$ whose birth index is $\mathbf{b}(e)=\left(b_{1}, b_{2}\right) \in \mathbb{Z}^{2}$. We say that the edge $e$ is negative if the number of connected components in $\mathcal{K}\left(b_{1}, b_{2}\right)$ is strictly less than that of $K\left(b_{1}, b_{2}-1\right)$. Otherwise, the edge $e$ is positive.

- Given a simplicial complex $K$ and $k \in \mathbb{Z}_{\geq 0}$, let $C_{k}(K)$ be the $k$-th chain group of $K$, i.e. the $\mathbb{F}$-vector space freely generated by $k$-simplices in $K$. For $k \in \mathbb{Z}_{\geq 0}$, let $\partial_{k}: C_{k}(K) \rightarrow$ $C_{k-1}(K)$ be the boundary map, and $Z_{k}(K):=\operatorname{ker}\left(\partial_{k}\right)$ the $k$-th cycle group of $K$.

- Let $\mathcal{K}: \mathbb{Z}^{2} \rightarrow$ Simp be a filtration. For each $k \in \mathbb{Z}_{\geq 0}$, let $C_{k}(\mathcal{K}): \mathbb{Z}^{2} \rightarrow$ Vec be the module defined as $C_{k}(\mathcal{K})(\mathbf{a}):=C_{k}(\mathcal{K}(\mathbf{a}))$, where the internal maps $\varphi_{\mathcal{K}}(\mathbf{a}, \mathbf{b})$ are the canonical inclusion maps $C_{k}(\mathcal{K}(\mathbf{a})) \hookrightarrow C_{k}(\mathcal{K}(\mathbf{b}))$. In particular, if $\mathcal{K}$ is 1-critical, then $C_{k}(\mathcal{K})$ is the free module whose basis elements one-to-one correspond to all the $k$-th simplices in $S$. More specifically, the birth of a simplex $\sigma \in S$ in $\mathcal{K}$ at $\mathbf{a} \in \mathbb{Z}^{d}$ corresponds to a generator of $C_{k}(\mathcal{K})$ at a.

Theorem 5.2. Let $\mathcal{K}$ be the 1-skeleton of the $\mathbb{Z}^{2}$-indexed Rips filtration of an aug-MS. Let $\mathcal{K}^{-}$be the filtration of $\mathcal{K}$ that is obtained by removing all positive edges in $\mathcal{K}$. Then,

(i) The following sequence of persistence modules is exact:

$$
0 \rightarrow Z_{1}\left(\mathcal{K}^{-}\right) \stackrel{i}{\rightarrow} C_{1}\left(\mathcal{K}^{-}\right) \stackrel{\partial_{1}}{\rightarrow} C_{0}\left(\mathcal{K}^{-}\right) \stackrel{p}{\rightarrow} \mathrm{H}_{0}(\mathcal{K}) \rightarrow 0,
$$

where $i$ is the canonical inclusion, $\partial_{1}$ is the boundary map, $p$ is the canonical projection.

(ii) The sequence in (12) is a minimal free resolution of $\mathrm{H}_{0}(\mathcal{K}){ }^{4}$

We prove Theorem 5.2 at the end of this section. For example, consider the aug-MS $\mathcal{X}$ in Figure 1 (A). We can read off the graded Betti number of $\mathrm{H}_{0}\left(\mathcal{R}_{\bullet}^{\mathrm{bi}}(\mathcal{X})\right): \mathbb{R}^{2} \rightarrow$ Vec from $\mathcal{R}_{\bullet}^{\text {bi }}(\mathcal{X})$. See Figure 11.

\footnotetext{
${ }^{3} d_{X}^{\mathbb{Z}}$ does not necessarily satisfy the triangle inequality, but it does not prevent from defining $\mathcal{R}_{\bullet}^{\mathrm{bi}}\left(X, d_{X}^{\mathbb{Z}}, f_{X}^{\mathbb{Z}}\right)$.

${ }^{4}$ This means that $F^{0}=C_{0}\left(\mathcal{K}^{-}\right), F^{1}=C_{1}\left(\mathcal{K}^{-}\right), F^{2}=Z_{1}\left(\mathcal{K}^{-}\right)$and $F^{i}=0$ for $i>2$ in the chain of (1).
} 


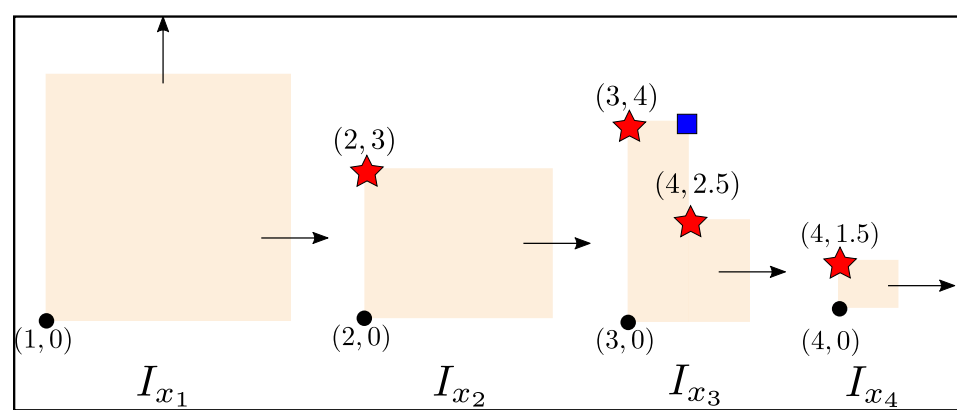

$(A)$

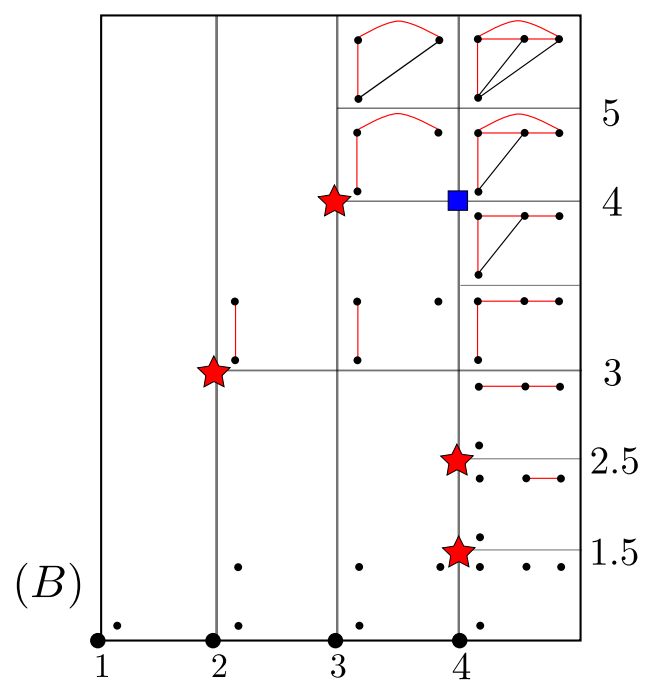

Figure 11: (A) The ER-staircode $\mathcal{I}_{\mathcal{X}}$ of $\mathcal{X}$ in Figure 1 (A). The types of corner points are indicated by circles (0-th), stars (1-st), and squares (2-nd). (B) The 1-skeleton of $\mathcal{K}:=\mathcal{R}_{\bullet}^{\mathrm{bi}}(\mathcal{X})$. Red edges and black edges are negative and positive, respectively. The four generators of $C_{0}(\mathcal{K})$ are located at grades $(1,0),(2,0),(3,0),(4,0)$, forming the support of the zeroth graded Betti number of $\mathrm{H}_{0}(\mathcal{K})$ (marked by circles). The birth grades of four negative edges are $(2,3),(3,4),(4,1.5)$, and $(4,2.5)$, forming the support of the first graded Betti number (marked by stars). The unique cycle consisting solely of negative edges is $x_{2} x_{3}+x_{3} x_{4}+x_{4} x_{2}$, which is born at $(4,4)$, the unique support point of the second graded Betti number. Observe that the locations of corner points in $\mathcal{I}_{\mathcal{X}}$ one-to-one correspond to the support of graded Betti numbers of $\mathrm{H}_{0}(\mathcal{K})$ which illustrates that Theorem 5.4 holds. 
The ER-staircode and the graded Betti numbers. Next we will see that for any aug-MS $\mathcal{X}$, the graded Betti numbers of the zeroth homology of $\mathcal{R}_{\bullet}^{\text {bi }}(\mathcal{X})$ can be extracted from the ER-staircode of $\mathcal{X}$.

Given finite $M: \mathbb{Z}^{2} \rightarrow$ Vec, the support of the $i$-th graded Betti number $\beta_{i}^{M}$ of $M$ is defined as $\operatorname{supp}\left(\beta_{i}^{M}\right):=\left\{\mathbf{a} \in \mathbb{Z}^{2}: \beta_{i}^{M}(\mathbf{a}) \neq 0\right\}$. Theorem 5.2 directly implies:

Lemma 5.3. Let $\mathcal{K}$ be the $\mathbb{Z}^{2}$-indexed Rips filtration of an aug-MS and let $M:=\mathrm{H}_{0}(\mathcal{K})$. For each $i=0,1,2, \beta_{i}^{M}(\mathbf{a}) \leq 1, \mathbf{a} \in \mathbb{Z}^{2}$ and for every pair $i \neq j$ in $\{0,1,2\}, \operatorname{supp}\left(\beta_{i}^{M}\right) \cap$ $\operatorname{supp}\left(\beta_{j}^{M}\right)=\emptyset$

Proof. Since we concern the zeroth homology of $\mathcal{K}$, let us assume that $\mathcal{K}$ itself consists solely of vertices and edges. By Theorem 5.2, it suffices to show that every generator of $Z_{1}\left(\mathcal{K}^{-}\right)$, $C_{1}\left(\mathcal{K}^{-}\right)$, and $C_{0}\left(\mathcal{K}^{-}\right)$is born at a different grade. In $C_{0}\left(\mathcal{K}^{-}\right)$, every vertex $x_{i}$ is born at $(i, 0)$ for $i=1, \ldots, n$. Therefore, $\beta_{0}^{M}(\mathbf{a}) \leq 1$ for every $\mathbf{a} \in \mathbb{Z}^{2}$ and $\operatorname{supp}\left(\beta_{0}^{M}\right) \subset \mathbb{Z} \times\{0\}$. Also, by Definition 5.1, every generator of $C_{1}\left(\mathcal{K}^{-}\right)$and $Z_{1}\left(\mathcal{K}^{-}\right)$is born at different grade in $\mathbb{Z} \times \mathbb{N}$, completing the proof.

Given any two functions $\alpha, \alpha^{\prime}: \mathbb{Z}^{2} \rightarrow \mathbb{Z}_{\geq 0}$, we define $\alpha-\alpha^{\prime}: \mathbb{Z}^{2} \rightarrow \mathbb{Z}_{\geq 0}$ as

$$
\left(\alpha-\alpha^{\prime}\right)(\mathbf{x})=\max \left(\alpha(\mathbf{x})-\alpha^{\prime}(\mathbf{x}), 0\right), \text { for } \mathbf{x} \in \mathbb{Z}^{2} .
$$

Theorem 5.4. Let $\mathcal{K}$ be the $\mathbb{Z}^{2}$-indexed Rips filtration of an aug- $M S \mathcal{X}$ and let $M:=\mathrm{H}_{0}(\mathcal{K})$. Let $\beta_{i}^{M}$ be the $i$-th grade Betti number of $M$. Then,

$$
\beta_{0}^{M}=\gamma_{0}^{\mathcal{X}}, \quad \beta_{1}^{M}=\gamma_{1}^{\mathcal{X}}-\gamma_{2}^{\mathcal{X}}, \quad \beta_{2}^{M}=\gamma_{2}^{\mathcal{X}}-\gamma_{1}^{\mathcal{X}} .
$$

In particular, we note that the elder-rule feature functions $\gamma_{j}^{\mathcal{X}}$ are easy to compute, as one only needs to compute and aggregate the type of each corner in staircase intervals in the ER-staircode of $\mathcal{X}$. Once $\gamma_{j}^{\mathcal{X}}$ s are known, one can easily compute the graded Betti number of $\mathrm{H}_{0}\left(\mathcal{R}_{\bullet}^{\text {bi }}(\mathcal{X})\right)$ by Theorem 5.4. See Example 5.5 below. We also remark that Koszul homology formulae [34, Proposition 5.1] are in a similar form to those in (13). However, Koszul homology formulae do not directly imply those in (13) nor vice versa.

Example 5.5 (Non-interval-decomposable case). Consider the metric space $\left(\left\{x_{i}\right\}_{i=1}^{4}, d_{X}\right)$ in Figure 1 (A). Define $h_{X}:\left\{x_{i}\right\}_{i=1}^{4} \rightarrow \mathbb{R}$ as $h_{X}\left(x_{1}\right)=1, h_{X}\left(x_{2}\right)=3, h_{X}\left(x_{3}\right)=2, h_{X}\left(x_{4}\right)=4$. For $\mathcal{X}:=\left(X, d_{X}, g_{X}\right)$, let $\mathcal{I}_{\mathcal{X}}:=\left\{I_{x_{i}}: i=1,2,3,4\right\}$ be the ER-staircode and let $M:=$ $\mathrm{H}_{0}\left(\mathcal{R}^{\mathrm{bi}}(\mathcal{X})\right)$ and $N:=\bigoplus_{i=1}^{4} I^{I_{x_{i}}}$. Utilizing Theorem 5.4, it it not hard to check that $\beta_{1}^{M} \neq \beta_{1}^{N}$ and $\beta_{2}^{M} \neq \beta_{2}^{N}$ (see Figure 12). Therefore, $M \neq N$ and thus, by Theorem 4.16, $M$ is not interval decomposable.

Proof of Theorem 5.4. Let $\mathcal{X}:=\left(X, d_{X}, f_{X}\right)$ with $X=\left\{x_{1}, \ldots, x_{n}\right\}$, and assume that $f_{X}\left(x_{1}\right)<\ldots<f_{X}\left(x_{n}\right)$. By the construction of $\mathcal{K}$ and $\gamma_{i}^{\mathcal{X}}$, it suffices to show the equalities in (13) hold on $\mathcal{A}:=\{1,2, \ldots, n\} \times\left\{0,1, \ldots,\left(\begin{array}{l}n \\ 2\end{array}\right)\right\} \subset \mathbb{Z}^{2}\left(\beta_{i}^{M}\right.$ and $\gamma_{i}^{\mathcal{X}}$ vanish outside $\mathcal{A}$ for $i=0,1,2)$. By Theorem 5.2 and the construction of $\gamma_{0}^{\mathcal{X}}$, both of $\beta_{0}^{M}$ and $\gamma_{0}^{\mathcal{X}}$ have values 1 on $\left.\mathcal{A}\right|_{y=0}=\{(1,0),(2,0),(3,0) \ldots,(n, 0)\}$ and zero outside $\left.\mathcal{A}\right|_{y=0}$, implying that $\beta_{0}^{M}=\gamma_{0}^{\mathcal{X}}$. Note that when $i=1,2$, the supports of $\beta_{i}^{M}$ and $\gamma_{i}^{\mathcal{X}}$ are contained in $\left.\mathcal{A}\right|_{y>0}=\{1,2, \ldots, n\} \times\left\{1, \ldots,\left(\begin{array}{l}n \\ 2\end{array}\right)\right\}$. Using induction on $x$-coordinate of $\mathbb{Z}^{2}$, we will prove 

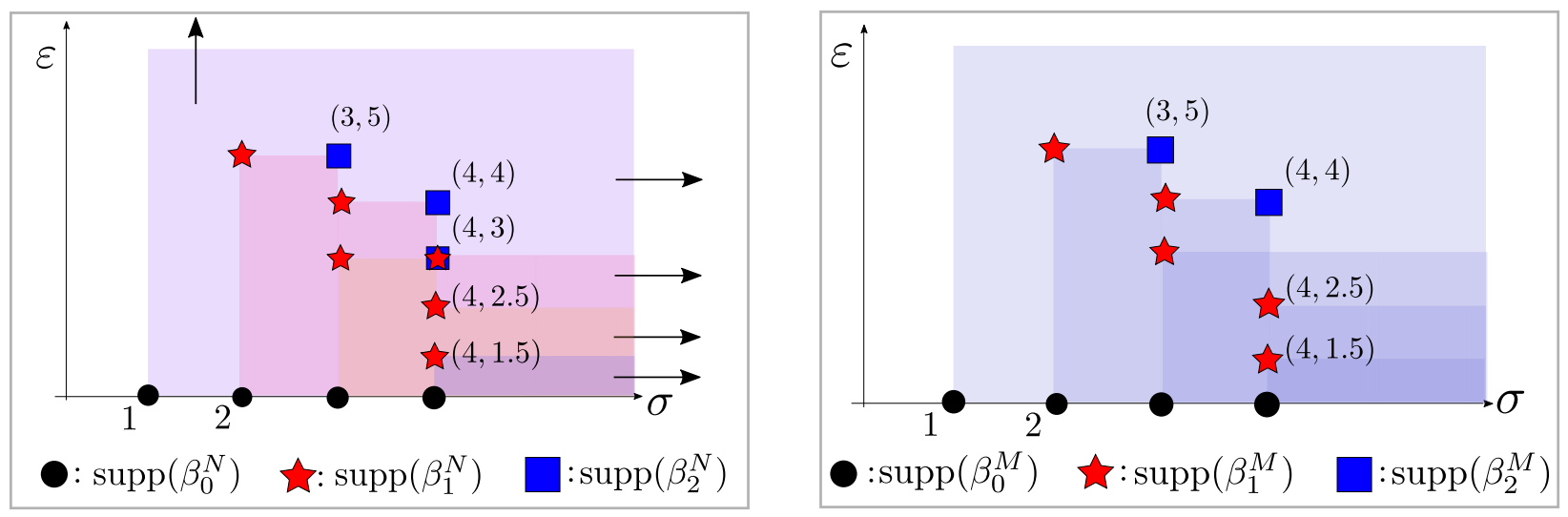

Figure 12: The respective supports of the graded Betti numbers of $N$ (left) and $M$ (right) from Example 5.5. All the graded Betti numbers attain the value 1 on their supports. The graded Betti numbers of $N$ are directly obtained by stacking the staircase intervals in the ER-staircode of $\mathcal{X}$ (Remarks 2.4 (i) and 3.10). The graded Betti numbers of $M$ are obtained by applying Theorem 5.4 to the graded Betti numbers of $N$; in particular the support-points of $\beta_{1}^{N}$ and $\beta_{2}^{N}$ at $(4,3)$ are canceled out.

that $\beta_{1}^{M}=\gamma_{1}^{\mathcal{X}}-\gamma_{2}^{\mathcal{X}}$ and $\beta_{2}^{M}=\gamma_{2}^{\mathcal{X}}-\gamma_{1}^{\mathcal{X}}$ on the horizontal line $\left.\mathcal{A}\right|_{y=1}=\{1,2, \ldots, n\} \times\{1\}$. Note that $\mathcal{K}(1, b)=\left\{\left\{x_{1}\right\}\right\}$ for all $1 \leq b \leq\left(\begin{array}{l}n \\ 2\end{array}\right)$, and thus again by Theorem 5.2 and the construction of $\gamma_{i}^{\mathcal{X}}, i=1,2$,

$$
\text { for } 1 \leq b \leq\left(\begin{array}{c}
n \\
2
\end{array}\right), \quad \beta_{1}^{M}(1, b)=\gamma_{1}^{\mathcal{X}}(1, b)=0 \text {, and } \beta_{2}^{M}(1, b)=\gamma_{2}^{\mathcal{X}}(1, b)=0 .
$$

Specifically, we have $\beta_{1}^{M}(1,1)=\gamma_{1}^{\mathcal{X}}(1,1)=\gamma_{1}^{\mathcal{X}}(1,1)-\gamma_{2}^{\mathcal{X}}(1,1)$ and $\beta_{2}^{M}(1,1)=\gamma_{2}^{\mathcal{X}}(1,1)=$ $\gamma_{2}^{\mathcal{X}}(1,1)-\gamma_{1}^{\mathcal{X}}(1,1)$. Fix a natural number $m>2$ and assume that $\beta_{1}^{M}(a, 1)=\gamma_{1}^{\mathcal{X}}(a, 1)-$ $\gamma_{2}^{\mathcal{X}}(a, 1)$ and $\beta_{2}^{M}(a, 1)=\gamma_{2}^{\mathcal{X}}(a, 1)-\gamma_{1}^{\mathcal{X}}(a, 1)$ for $1 \leq a \leq m-1$. By Theorem 4.9 and Theorem 2.5 in the appendix, we have: $\sum_{\mathbf{x} \leq(m, 1)} \sum_{i=0}^{2}(-1)^{i} \beta_{i}^{M}(\mathbf{x}) \stackrel{(*)}{=} \sum_{\mathbf{x} \leq(m, 1)} \sum_{i=0}^{2}(-1)^{i} \gamma_{i}^{\mathcal{X}}(\mathbf{x})$. Since (1) $\beta_{0}^{M}=\gamma_{0}^{\mathcal{X}}$ on the entire $\mathbb{Z}^{2}$, and (2) $\beta_{i}^{M}, \gamma_{i}^{\mathcal{X}}$ vanish outside $\mathcal{A}$ for $i=1,2$, the induction hypothesis reduces equality $(*)$ to

$$
-\beta_{1}^{M}(m, 1)+\beta_{2}^{M}(m, 1)=-\gamma_{1}^{\mathcal{X}}(m, 1)+\gamma_{2}^{\mathcal{X}}(m, 1) .
$$

By Lemma 5.3, three cases are possible: (Case 1) $\beta_{1}^{M}(m, 1)=1$ and $\beta_{2}^{M}(m, 1)=0$, (Case 2) $\beta_{1}^{M}(m, 1)=0$ and $\beta_{2}^{M}(m, 1)=1$, or (Case 3) $\beta_{1}^{M}(m, 1)=0$ and $\beta_{2}^{M}(m, 1)=0$. Invoking that $\gamma_{1}^{\mathcal{X}}(m, 1)$ and $\gamma_{2}^{\mathcal{X}}(m, 1)$ are non-negative, in all cases, we have

$$
\beta_{1}^{M}(m, 1)=\gamma_{1}^{\mathcal{X}}(m, 1)-\gamma_{2}^{\mathcal{X}}(m, 1), \quad \beta_{2}^{M}(m, 1)=\gamma_{2}^{\mathcal{X}}(m, 1)-\gamma_{1}^{\mathcal{X}}(m, 1),
$$

completing the proof of $\beta_{1}^{M}=\gamma_{1}^{\mathcal{X}}-\gamma_{2}^{\mathcal{X}}$ and $\beta_{2}^{M}=\gamma_{2}^{\mathcal{X}}-\gamma_{1}^{\mathcal{X}}$ on $\left.\mathcal{A}\right|_{y=1}$. We next apply the same strategy to the horizontal lines $y=2, \ldots, y=\left(\begin{array}{l}n \\ 2\end{array}\right)$ in order, completing the proof.

Remark 5.6 (Theorem 5.4 for non-generic cases). Consider an aug-MS $\mathcal{X}=\left(X, d_{X}, f_{X}\right)$ such that $\mathcal{X}$ is not generic. Then we pick a total order $<$ on $X$ and another total order $\prec$ on the collection of all pairs $x_{i}, x_{j}\left(x_{i} \neq x_{j}\right)$ in $X$, which are compatible with $f_{X}$ and $d_{X}$, 

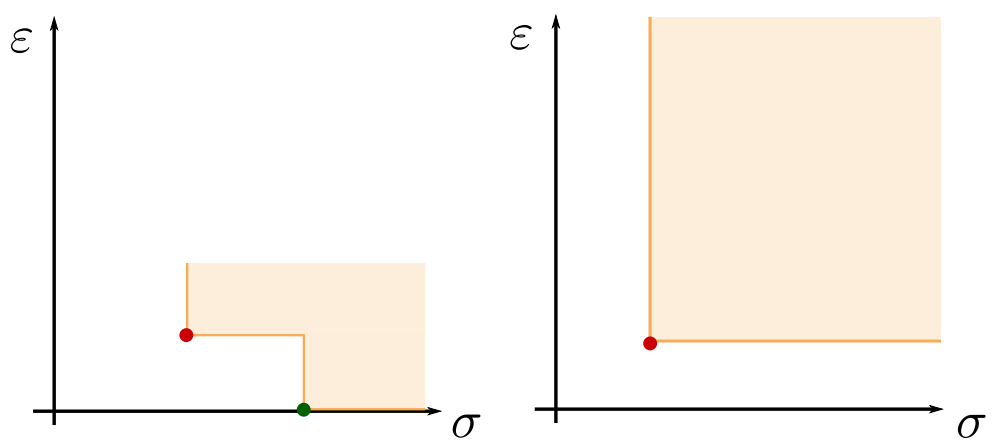

Figure 13: If an interval $J$ that is contained in the quadrant $U(1,0)$ is either [not a staircase] or [a staircase whose minimum is not in the $\sigma$-axis], then there exists a point $\mathbf{a}$ in the interior of $U(0,0)$ such that $\beta_{0}^{I^{J}}(\mathbf{a})=1$ (red points in the figure above).

respectively. This gives rise to the injective function $f_{X}^{\mathbb{Z}}$ and the pairwise-distinct-distance $d_{X}^{\mathbb{Z}}$ on $X$ as in Definition 5.1. The unique ER-staircode of $\mathcal{X}^{\mathbb{Z}}=\left(X, d_{X}^{\mathbb{Z}}, f_{Z}^{\mathbb{Z}}\right)$ recovers the graded Betti numbers of $\left.\mathrm{H}_{0}\left(\mathcal{R}_{\bullet}^{\text {bi }}\left(\mathcal{X}^{\mathbb{Z}}\right)\right)\right|_{\mathbb{Z}^{2}}: \mathbb{Z}^{2} \rightarrow$ Vec by Theorem 5.4.

Below, we will make use of Theorem 5.4 in proving Theorem 4.16.

Proof of Theorem 4.16. Without loss of generality, let us assume that $X=\left\{x_{1}, x_{2}, \ldots, x_{n}\right\}$ with $f_{X}\left(x_{i}\right)=i$ for $i=1,2, \ldots, n$. Also, let $M \cong \bigoplus_{k \in K} I^{J_{k}}$ for some indexing set $K$. Observe that $M$ is upper-right continuous, i.e. for each $\left(\sigma_{0}, \varepsilon_{0}\right) \in \mathbb{R}^{2}$, there exist $e_{1}, e_{2}>0$ s.t. if $\sigma_{0} \leq \sigma \leq \sigma_{0}+e_{1}$ and $\varepsilon_{0} \leq \varepsilon \leq \varepsilon_{0}+e_{2}$, then $M_{\left(\sigma_{0}, \varepsilon_{0}\right)}=M_{(\sigma, \varepsilon)}$. Hence, the lower-left boundary ${ }^{5}$ of each $J_{k}$ belongs to $J_{k}$. Also, note that $M_{(\sigma, \varepsilon)} \neq 0$ if and only if $(\sigma, \varepsilon) \in U(1,0)$.

Claim $1 \quad[\operatorname{barc}(M)$ consists of $n$ staircase intervals (Definition 3.4) and their minimal elements are $(1,0),(2,0), \ldots,(n, 0)$.] First, let us show that each interval in $\operatorname{barc}(M)$ is a staircase whose minimal element lies on the $\sigma$-axis. Suppose not, i.e. there exists $k_{0} \in K$ s.t. $J_{k_{0}}$ is either [not a staircase] or [a staircase whose minimum is not in the $\sigma$-axis]. Either implies that $J_{k_{0}}$ contains a minimal element a in the interior of $U(0,0)$ (see Figure 13). Then, since $M \cong \bigoplus_{k \in K} I^{J_{k}}$, by Remark 2.4 (i), we have

$$
1=\beta_{0}^{I^{J_{k_{0}}}}(\mathbf{a}) \leq \sum_{k \in K} \beta_{0}^{I^{J_{k}}}(\mathbf{a})=\beta_{0}^{M}(\mathbf{a}) .
$$

However, by Theorem 5.4, we have

$$
\operatorname{supp}\left(\beta_{0}^{M}\right)=\{(i, 0): i=1, \ldots, n\} \not \ngtr \mathbf{a},
$$

a contradiction. Therefore, (1) each $J_{k}$ has its minimum element in the $\sigma$-axis, and (2) since $\beta_{0}^{M}=\sum_{k \in K} \beta_{0}^{I^{J_{k}}}$, by invoking Remark 3.10, the minimums of $J_{k} \mathrm{~s}$ form the set $\{(i, 0): i=$ $1, \ldots, n\}$. This implies that the indexing set $K$ contains $n$ elements, as desired.

From now on, we denote $\operatorname{barc}(M)$ by $\left\{J_{k}\right\}_{k=1}^{n}$ where the minimum of $J_{k}$ is $(k, 0)$ for each $k$. Also, let

$$
\varepsilon_{1}:=\max _{x_{i}, x_{j} \in X} d_{X}\left(x_{i}, x_{j}\right) .
$$

\footnotetext{
${ }^{5}(\sigma, \varepsilon) \in \mathbb{R}^{2}$ is a lower-left boundary point of $J_{k}$ if $(\sigma, \varepsilon)$ belongs to the boundary of $J_{k}$ and for any $r>0$, $(\sigma-r, \varepsilon-r) \notin J_{k}$. The set of lower-left boundary points of $J_{k}$ is called the lower-left boundary of $J_{k}$.
} 
Claim $2 \quad\left[J_{1}=U(1,0).\right]$ Observe that, if $\sigma \in[n, \infty)$ and $\varepsilon \in\left[\varepsilon_{1}, \infty\right)$, then

$$
\operatorname{dim} M_{(\sigma, \varepsilon)}=1 \quad \text { and } \operatorname{rank} \varphi_{M}((1,0),(\sigma, \varepsilon))=1 .
$$

Since rank $\varphi_{M}((1,0),(\sigma, \varepsilon))$ is equal to the total multiplicity of elements of $\operatorname{barc}(M)$ which contain both $(1,0)$ and $(\sigma, \varepsilon), J_{1}$ must be $U(1,0)$.

Now let $f: \bigoplus_{k=1}^{n} I^{J_{k}} \rightarrow M$ be any isomorphism. For each $k$, let $1_{k}:=1 \in\left(I^{J_{k}}\right)_{(k, 0)}(=\mathbb{F})$, and let $f_{(k, 0)}\left(1_{k}\right):=v_{k} \in M_{(k, 0)}$. For $x_{k} \in X$ and $(\sigma, \varepsilon) \in[k, \infty) \times \mathbb{R}_{+}$, let $\left[x_{k}\right]_{(\sigma, \varepsilon)}$ be the zeroth homology class of $x_{k}$. When confusion is unlikely, we will suppress the subscript $(\sigma, \varepsilon)$ in $\left[x_{k}\right]_{(\sigma, \varepsilon)}$.

Note that, by the definition of $M_{(k, 0)}$ for each $k=1, \ldots, n$, there exist $c_{k \ell} \in \mathbb{F}$ for $\ell=1, \ldots, k$ s.t.

$$
\begin{aligned}
v_{1} & =c_{11}\left[x_{1}\right] \\
v_{2} & =c_{21}\left[x_{1}\right]+c_{22}\left[x_{2}\right] \\
\vdots & \\
v_{n} & =c_{n 1}\left[x_{1}\right]+\ldots+c_{n n}\left[x_{n}\right] .
\end{aligned}
$$

An $x_{\ell} \in X$ will be called a summand of $v_{k}$ if $c_{k l} \neq 0$. Also, for each $k$, we define the function $\mathbf{v}_{k}: U(k, 0) \rightarrow \coprod_{(\sigma, \varepsilon) \in U(k, 0)} M_{(\sigma, \varepsilon)}$ as $(\sigma, \varepsilon) \mapsto \varphi_{M}((k, 0),(\sigma, \varepsilon))\left(v_{k}\right)$. Let $\operatorname{supp}\left(\mathbf{v}_{k}\right)$ be the set of $(\sigma, \varepsilon) \in U(k, 0)$ s.t. $\mathbf{v}_{k}(\sigma, \varepsilon)$ is nonzero in $M_{(\sigma, \varepsilon)}$. Since $f$ is an isomorphism, we have:

(i) $\left\{\operatorname{supp}\left(\mathbf{v}_{k}\right)\right\}_{k=1}^{n}=\left\{J_{k}\right\}_{k=1}^{n}$,

(ii) For each $(\sigma, \varepsilon) \in U(1,0),\left\{\mathbf{v}_{k}(\sigma, \varepsilon): \sigma \in[k, \infty)\right\}$ is a basis of $M_{(\sigma, \varepsilon)}$.

Now we investigate constraints on the coefficients $c_{k \ell}$.

Claim 3 [For each $k, x_{k}$ is a summand of $v_{k}$.] By item (ii) above, the set

$$
B_{k}:=\left\{\mathbf{v}_{1}(k, 0), \mathbf{v}_{2}(k, 0), \ldots, \mathbf{v}_{k}(k, 0)\right\}
$$

is linearly independent in $M_{(k, 0)}$. Invoking equations in (16) and the definition of $\mathbf{v}_{k}$, observe that if $c_{k k}=0$, then $B_{k}$ is linearly dependent, a contradiction.

Claim 4 [For $k \in\{2, \ldots, n\}, \sum_{\ell=1}^{k} c_{k \ell}=0$ and $v_{k}$ has at least two summands.] Fix $k \in\{2, \ldots, n\}$ and pick any $(\sigma, \varepsilon) \in U\left(n, \varepsilon_{1}\right)$ (see $\left.(15)\right)$. Then, we have $\left[x_{\ell_{1}}\right]_{(\sigma, \varepsilon)}=\left[x_{\ell_{2}}\right]_{(\sigma, \varepsilon)}$ for all $\ell_{1}, \ell_{2} \in\{1, \ldots, n\}$, and thus $\mathbf{v}_{k}(\sigma, \varepsilon)=\left(\sum_{\ell=1}^{k} c_{k \ell}\right) \cdot\left[x_{k}\right]_{(\sigma, \varepsilon)}$. Note that $1=\operatorname{dim} M_{(\sigma, \varepsilon)}$, which is equal to the number of intervals in $\operatorname{barc}(M)$ that includes $(\sigma, \varepsilon)$. Since $U(1,0) \in$ $\operatorname{barc}(M)$ includes $(\sigma, \varepsilon)$ (Claim 2), $\operatorname{supp}\left(\mathbf{v}_{k}\right)$ must not include $(\sigma, \varepsilon)$, which implies $\sum_{\ell=1}^{k} c_{k \ell}$ to be 0 . This also forces $v_{k}$ to admit at least two different summands, including $x_{k}$ (Claim $3)$.

Recall that, for each $k, I_{x_{k}}$ denotes the elder-rule interval associated to $x_{k}$ (see $\left.(2)\right)$. 
Claim 5 [For each $\left.k, I_{x_{k}} \subseteq \operatorname{supp}\left(\mathbf{v}_{k}\right)\right]$ By Claim 2, item (i) above, and Definition 3.3, we readily know $I_{x_{1}}=\operatorname{supp}\left(\mathbf{v}_{1}\right)=U(1,0)$. Let us fix any $k \in\{2, \ldots, n\}$ and any $(\sigma, \varepsilon) \in I_{x_{k}}$. By definition of $I_{x_{k}},\left[x_{k}\right]_{(\sigma, \varepsilon)}$ is the singleton $\left\{x_{k}\right\}$. Therefore, in $\mathbf{v}_{k}(\sigma, \varepsilon)=\sum_{\ell=1}^{k} c_{k \ell}\left[x_{\ell}\right]_{(\sigma, \varepsilon)}$, the nontrivial term $c_{k k}\left[x_{k}\right]_{(\sigma, \varepsilon)}$ cannot be combined with any other term (By Claim $3, c_{k k} \neq 0$, and by Claim 4, there is another nonzero $c_{k \ell}$ ). This implies that $\mathbf{v}_{k}(\sigma, \varepsilon) \neq 0$, and in turn $(\sigma, \varepsilon) \in \operatorname{supp}\left(\mathbf{v}_{k}\right)$.

By Claim 5, we have:

$$
\operatorname{dm}(M)=\sum_{k=1}^{n} \mathbb{1}_{I_{x_{k}}} \leq \sum_{k=1}^{n} \mathbb{1}_{\operatorname{supp}\left(\mathbf{v}_{k}\right)}=\operatorname{dm}(M) .
$$

This implies that for each $k, \mathbb{1}_{I_{x_{k}}}=\mathbb{1}_{\operatorname{supp}\left(\mathbf{v}_{k}\right)}$ and in turn $I_{x_{k}}=\operatorname{supp}\left(\mathbf{v}_{k}\right)=J_{k}$ by item (i) above.

Proof of Theorem 5.2. In order to prove Theorem 5.2, we need the two lemmas below.

Lemma 5.7. Let $\mathcal{K}: \mathbb{Z}^{2} \rightarrow$ Simp be the 1-skeleton of the $\mathbb{Z}^{2}$-indexed Rips filtration of an aug-MS. Let $K^{-}$be the filtration of $\mathcal{K}$ that is obtained by removing all positive edges in $\mathcal{K}$. Then, $\mathrm{H}_{0}(\mathcal{K}) \cong \mathrm{H}_{0}\left(\mathcal{K}^{-}\right)$.

Proof. Observe that, for each $\mathbf{a} \in \mathbb{Z}^{2}$, it holds that $\pi_{0}(\mathcal{K}(\mathbf{a}))=\pi_{0}\left(\mathcal{K}^{-}(\mathbf{a})\right) \in \operatorname{Subpart}(X)$. Therefore, the two bipersistence treegrams $\pi_{0}(\mathcal{K}), \pi_{0}\left(\mathcal{K}^{-}\right): \mathbb{Z}^{2} \rightarrow \operatorname{Subpart}(X)$ are the same. By Proposition 4.15, we have $\mathrm{H}_{0}(\mathcal{K}) \cong \mathcal{F}_{\mathbb{F}} \circ \pi_{0}(\mathcal{K}) \cong \mathcal{F}_{\mathbb{F}} \circ \pi_{0}\left(\mathcal{K}^{-}\right) \cong \mathrm{H}_{0}\left(\mathcal{K}^{-}\right)$.

Lemma 5.8. For any simplicial 1-complex, the following sequence is exact

$$
0 \rightarrow Z_{1}(K) \stackrel{i}{\rightarrow} C_{1}(K) \stackrel{\partial_{1}}{\rightarrow} C_{0}(K) \stackrel{p}{\rightarrow} \mathrm{H}_{0}(K) \rightarrow 0
$$

where $i$ is the canonical inclusion, $\partial_{1}$ is the boundary map, and $p$ is the canonical quotient.

The proof is straightforward and thus we omit it.

For a persistence module $M$, let $I M$ denote the submodule of $M$ that is generated by the images of all linear maps $\varphi_{N}(\mathbf{a}, \mathbf{b})$, with $\mathbf{a}<\mathbf{b}$ in $\mathbb{Z}^{2}$. We are now ready to prove Theorem 5.2 .

Proof of Theorem 5.2. Let us prove (i). By Lemma 5.7, $\mathrm{H}_{0}\left(\mathcal{K}^{-}\right)$is isomorphic to $\mathrm{H}_{0}(\mathcal{K})$ and thus it suffices to show the exactness of

$$
0 \rightarrow Z_{1}\left(\mathcal{K}^{-}\right) \stackrel{i}{\rightarrow} C_{1}\left(\mathcal{K}^{-}\right) \stackrel{\partial_{1}}{\rightarrow} C_{0}\left(\mathcal{K}^{-}\right) \stackrel{p}{\rightarrow} \mathrm{H}_{0}\left(\mathcal{K}^{-}\right) \rightarrow 0
$$

At each grade $\mathbf{a} \in \mathbb{Z}^{2}$, we have the sequence of vector spaces and linear maps:

$$
0 \rightarrow Z_{1}\left(\mathcal{K}_{\mathbf{a}}^{-}\right) \stackrel{i_{\mathbf{a}}}{\rightarrow} C_{1}\left(\mathcal{K}_{\mathbf{a}}^{-}\right) \stackrel{\left(\partial_{1}\right)_{\mathbf{a}}}{\rightarrow} C_{0}\left(\mathcal{K}_{\mathbf{a}}^{-}\right) \stackrel{p_{\mathbf{a}}}{\rightarrow} \mathrm{H}_{0}\left(\mathcal{K}_{\mathbf{a}}^{-}\right) \rightarrow 0
$$

which is exact by Lemma 5.8 .

Next, we prove (ii). In the following proof, we assume the ground field $\mathbb{F}$ is $\mathbb{Z}_{2}$ for the sake of simplicity. We need to show that (a) $C_{0}\left(\mathcal{K}^{-}\right), C_{1}\left(\mathcal{K}^{-}\right)$, and $Z_{1}\left(\mathcal{K}^{-}\right)$are free modules, and that (b) the sequence in (12) satisfies the minimality condition. Let us prove (a). By 
definition, it is clear that $C_{0}\left(\mathcal{K}^{-}\right)$and $C_{1}\left(\mathcal{K}^{-}\right)$are free. Also, $Z_{1}\left(\mathcal{K}^{1}\right)$, the kernel of $\partial_{1}$, is free by $[15$, Section 6$] .{ }^{6}$ Let us check (b). We show that the image of $C_{1}\left(\mathcal{K}^{-}\right)$via $\partial_{1}$ is contained in $I C_{0}\left(\mathcal{K}^{-}\right)$. It suffices to show that every generator of $C_{1}\left(\mathcal{K}^{-}\right)$is mapped into $I C_{0}\left(\mathcal{K}^{-}\right)$. Pick any edge $x_{i} x_{j}(i<j)$ that appears in $\mathcal{K}^{-}$. Then, in the filtration $\mathcal{K}^{-}, x_{i} x_{j}$ is born at $\left(j, d_{X}^{\mathbb{Z}}\left(x_{i}, x_{j}\right)\right)=$ : a, whereas the vertices $x_{i}$ and $x_{j}$ are born at $(i, 0)$ and $(j, 0)$, respectively. Note that $(i, 0)<(j, 0)<\mathbf{a}$ in $\mathbb{Z}^{2}$. Therefore, $\left.\partial_{1}\right|_{\mathbf{a}}\left(x_{i} x_{j}\right)=x_{i}+x_{j} \in I C_{0}\left(\mathcal{K}^{-}\right)_{\mathbf{a}}$.

Let us show that the image of $Z_{1}\left(\mathcal{K}^{-}\right)$is contained in $I C_{1}\left(\mathcal{K}^{-}\right)$. To this end, it suffices to show that every generator of $Z_{1}\left(\mathcal{K}^{-}\right)$is mapped into $I C_{1}\left(\mathcal{K}^{-}\right)$. Suppose that a cycle

$$
c \stackrel{(*)}{=} x_{i_{1}} x_{j_{1}}+x_{i_{2}} x_{j_{2}}+\ldots+x_{i_{l}} x_{j_{l}}
$$

is born at grade $\mathbf{b}=\left(b_{1}, b_{2}\right) \in \mathbb{Z}^{2}$ in $\mathcal{K}^{-}$. Consider the vertical restriction $\left.\mathcal{K}^{-}\right|_{x=b_{1}}:\left\{b_{1}\right\} \times$ $\mathbb{Z} \rightarrow$ Simp. By the construction of $\mathcal{K}$ (Definition 5.1), at most one simplex can be added at each grade in $\left.\mathcal{K}^{-}\right|_{x=b_{1}}$ as index increases. Thus, there exists a unique edge on the RHS of equality $(*)$ which appears at $\mathbf{b}$ in $\left.\mathcal{K}^{-}\right|_{x=b_{1}}$ (if no edge is born at $\mathbf{b}$, then $c$ cannot be born at $\mathbf{b}$, contradicting the assumption). Without loss of generality, let $x_{i_{l}} x_{j_{l}}$ be such edge. We will show that $x_{i_{l}} x_{j_{l}}$ is not born at $\mathbf{b}$ in $\mathcal{K}^{-}$by contradiction. Suppose that $x_{i_{l}} x_{j_{l}}$ is born at $\mathbf{b}$ in $\mathcal{K}^{-}$. This implies that $x_{i_{l}} x_{j_{l}}$ is born at $\mathbf{b}$ in $\mathcal{K}\left(\supset \mathcal{K}^{-}\right)$, generating the cycle $c$. This means that $x_{i_{l}} x_{j_{l}}$ is positive, contradicting the fact that $x_{i_{l}} x_{j_{l}}$ is taken in the filtration $\mathcal{K}^{-}$ whose edges are negative in $\mathcal{K}$. Therefore, every edge on the RHS of equality $(*)$ is born at grades strictly smaller than $\mathbf{a}$. This implies that $\left.\partial_{1}\right|_{\mathbf{b}}(c) \in I C_{1}\left(\mathcal{K}^{-}\right)_{\mathbf{b}}$, as desired.

\section{Computation and Algorithms}

\subsection{Algorithm}

Theorem 6.1. Let $\left(X, d_{X}, f_{X}\right)$ be a finite aug-MS with $n=|X|$.

(a) We can compute the ER-staircode $I_{\mathcal{X}}=\left\{I_{x}: x \in X\right\}$ in $O\left(n^{2} \log n\right)$ time. If $X \subset \mathbb{R}^{d}$ for a fixed $d$ and $d_{X}$ the Euclidean distance, the time can be improved to $O\left(n^{2} \alpha(n)\right)$, where $\alpha(n)$ is the inverse Ackermann function.

(b) Each $I_{x} \in I_{\mathcal{X}}$ has complexity $O(n)$. Given $I_{\mathcal{X}}$, we can compute zeroth fibered barcode $B^{L}$ for any line $L$ with positive slope in $O\left(\left|B^{L}\right| \log n\right)$ time where $\left|B^{L}\right|$ is the size of $B^{L}$.

(c) Given $I_{\mathcal{X}}$, we can compute the zeroth graded Betti numbers in $O\left(n^{2}\right)$ time.

We sketch the proof of the above theorem in the remainder of this section, with missing details in Appendix C.

Consider a function value $\sigma \in \mathbb{R}$, and recall that $X_{\sigma}$ consists of all points in $X$ with $f_{X}$ value at most $\sigma$. Let $\mathcal{K}_{\sigma}=\mathcal{R}_{\bullet}\left(X_{\sigma}, d_{X}\right)$ denote the Rips filtration of $\left(X_{\sigma}, d_{X}\right)$ (recall Remark 4.2). The corresponding 1-parameter treegram (dendrogram) is $\theta_{\sigma}:=\pi_{0}\left(\mathcal{K}_{\sigma}\right)$. On the other hand, for any $\sigma$, we can consider the complete weighted graph $G_{\sigma}=\left(V_{\sigma}=X_{\sigma}, E_{\sigma}\right)$ with edge weight $w\left(x, x^{\prime}\right)=d_{X}\left(x, x^{\prime}\right)$ for any $x, x^{\prime} \in X_{\sigma}$. It is folklore that the treegram $\theta_{\sigma}$ can be computed from the minimum spanning tree (MST) $T_{\sigma}$ of $G_{\sigma}$.

\footnotetext{
${ }^{6}$ The authors of [15] observe that for any two free modules $M, N: \mathbb{Z}^{2} \rightarrow$ Vec, the kernel of any natural transformation $f: M \rightarrow N$ is a free module.
} 
Table 1: Complexity Comparison with [33] and [34] for computing the fibered barcode and graded Betti number of $\mathrm{H}_{0}(\mathcal{K})$, where $\mathcal{K}$ is the 1-skeleton $\mathbb{Z}^{2}$-indexed of Rips bifiltration of an aug-MS of $n$ points. $\left|B^{L}\right|$ is the cardinality of the fibered barcode for query line $L$ of positive slope.

\begin{tabular}{lccc}
\hline & Our Algorithm & RIVET [33] & Graded Betti number [34] \\
\hline Size of descriptor & $O\left(n^{2}\right)$ & $O\left(n^{6}\right) \sim O\left(n^{8}\right)$ & $\Omega\left(n^{2}\right)$ \\
Fibered barcodes query time & $O\left(\left|B^{L}\right| \log n\right)$ & $O\left(\log n+\left|B^{L}\right|\right)$ & - \\
Computation time & $O\left(n^{2} \log n\right)$ & $O\left(n^{8}\right) \sim O\left(n^{9}\right)$ & $\Omega\left(n^{3}\right)$ \\
\hline
\end{tabular}

Assume all points in $X$ are ordered $x_{1}, x_{2}, \ldots, x_{n}$ such that $f_{X}\left(x_{i}\right) \leq f_{X}\left(x_{j}\right)$ whenever $i<j$, and set $\sigma_{i}=f\left(x_{i}\right)$ for $i \in[1, n]$. Note that as $\sigma$ varies, $X_{\sigma}$ only changes at $\sigma_{i}$. For simplicity, we set $\theta_{i}:=\theta_{\sigma_{i}}=\pi_{0}\left(\mathcal{K}_{\sigma_{i}}\right), G_{i}:=G_{\sigma_{i}}$ and $T_{i}:=\operatorname{MST}\left(G_{i}\right)$ is the minimum spanning tree (MST) for the weighted graph $G_{i}$. Our algorithm depends on the following lemma, the proof of which is in Appendix C.2.

Lemma 6.2. A decorated ER-staircode for the finite aug-MS $\left(X, d_{X}, f_{X}\right)$ can be computed from the collection of treegrams $\left\{\theta_{i}, i \in[1, n]\right\}$ in $O\left(n^{2}\right)$ time.

In light of the above result, the algorithm to compute ER-staircode is rather simple:

(Step 1): We start with $T_{0}=$ empty tree. At the $i$-th iteration,

(Step 1-a) we update $T_{i-1}$ (already computed) to obtain $T_{i}$; and

(Step 1-b) compute $\theta_{i}$ from $T_{i}$ and $\theta_{i-1}$.

(Step 2): We use the approach described in the proof of Lemma 6.2 to compute the ERstaircode in $O\left(n^{2}\right)$ time.

For (Step 1-a), note that $G_{i}$ is obtained by inserting vertex $x_{i}$, as well as all $i-1$ edges between $\left(x_{i}, x_{j}\right), j \in[1, i-1]$, into graph $G_{i-1}$. By [19], one can update the minimum spanning tree $T_{i-1}$ of $G_{i-1}$ to obtain the MST $T_{i}$ of $G_{i}$ in $O(n)$ time.

For (Step 1-b), once all $i-1$ edges spanning $i$ vertices in $T_{i}$ are sorted, then we can easily build the treegram $\theta_{i}$ in $O(i \alpha(i))=O(n \alpha(n))$ time, by using union-find data structure (see Figure 16 in Appendix C.2). Sorting edges in $T_{i}$ takes $O(i \log i)=O(n \log n)$ time. Hence the total time spent on (Step 1-b) for all $i \in[1, n]$ is $O\left(n^{2} \log n\right)$.

We remark that knowing the order of all edges in $T_{i-1}$ may not help, as compared to $T_{i-1}, T_{i}$ may have $\Omega(i)$ different edges newly introduced, and these new edges still need to be sorted. Nevertheless, we show in Appendix C.1 that if $X \subset \mathbb{R}^{d}$ for a fixed dimension $d$, then each $T_{i}$ will only have constant number of different edges compared to $T_{i-1}$, and we can sort all edges in $T_{i}$ in $O(n)$ time by inserting the new edges to the sorted list of edges in $T_{i-1}$. Hence $\theta_{i}$ can be computed in $O(n \alpha(n))+O(n)=O(n \alpha(n))$ time for this case.

Putting everything together, Theorem 6.1 (a) follows. See Appendix C.1 for the proofs of (b) and (c). 


\subsection{Comparison with other algorithms}

Let $\mathcal{K}$ be the 1 -skeleton of the $\mathbb{Z}^{2}$-indexed Rips filtration of an aug-MS $\mathcal{X}=\left(X, d_{X}, f_{X}\right)$, where $|X|=n$. Let $M:=\mathrm{H}_{0}(\mathcal{K})$.

Comparison with [33]. Let $\kappa:=\kappa_{x} \kappa_{y}$, where $\kappa_{x}$ and $\kappa_{y}$ are the number of $x$ and $y$ coordinates in $\operatorname{supp}\left(\beta_{0}^{M}\right) \cup \operatorname{supp}\left(\beta_{1}^{M}\right)$, respectively. In our case $\kappa_{x}=n$ and

$$
\kappa_{y}=(\text { the number of negative edges in } \mathcal{K})
$$

which is between $O(n)$ and $O\left(n^{2}\right)$. Let $m$ be the number of simplices in $\mathcal{K}$, which is $O\left(n^{2}\right)$.

From the filtration $\mathcal{K}$, RIVET computes a certain data structure $\mathcal{A}^{\bullet}(M)$ of size $O\left(m \kappa^{2}\right)$ in $O\left(m^{3} \kappa+(m+\log \kappa) \kappa^{2}\right)$ time and $O\left(m^{2}+m \kappa^{2}\right)$ memory. This $\mathcal{A}^{\bullet}(M)$ allows efficient query about the fibered barcode of $M$ in $O\left(\log \kappa+\left|B^{L}\right|\right)$ where $\left|B^{L}\right|$ is the size of the fibered barcode $\operatorname{barc}\left(\left.M\right|_{L}\right)$ for a positive slope line $L \in \mathcal{L}$.

See Table 1 for the comparison of computational complexity between RIVET and our method.

Comparison with [34]. The algorithm in [34] takes as input a short chain complex of free modules $F^{2} \stackrel{\partial^{2}}{\longrightarrow} F^{1} \stackrel{\partial^{1}}{\longrightarrow} F^{0}$ such that $M \cong \operatorname{ker} \partial^{1} /$ im $\partial^{2}$ and outputs a minimal presentation of a 2-parameter persistence module $M$, from which the graded Betti numbers of $M$ are readily computed. It runs in time $O\left(\sum_{i}\left|F^{i}\right|^{3}\right)$ and requires $O\left(\sum_{i}\left|F^{i}\right|^{2}\right)$ memory, where $\left|F^{i}\right|$ denotes the size of a basis of $F^{i}$. In our setting, we readily have $\left|F^{0}\right|=0,\left|F^{1}\right|=n$, $\left|F^{2}\right|=$ (the number of negative edges in $\mathcal{K}$ ) which is between $O(n)$ and $O\left(n^{2}\right)$. Therefore, in order to obtain the graded Betti numbers via the method in [34], it takes at least $\Omega\left(n^{3}\right)$ time and $\Omega\left(n^{2}\right)$ memory.

\section{Discussion}

Some open questions and conjectures follow:

1. Barcodes and elder-rule-staircodes. (1) Let $\mathcal{X}=\left(X, d_{X}, f_{X}\right)$ be an aug-MS. If $x \in X$ has a constant conqueror, is the interval module supported by $I_{x}$ in (2) a summand of $\mathrm{H}_{0}\left(\mathcal{R}^{\text {bi }}(\mathcal{X})\right)$ ? (2) By virtue of Theorem 4.16, if $\mathrm{H}_{0}\left(\mathcal{R}^{\text {bi }}(\mathcal{X})\right)$ is interval decomposable, then the ER-staircode is identical to the generalized persistence diagram of $\mathrm{H}_{0}\left(\mathcal{R}^{\mathrm{bi}}(\mathcal{X})\right)$ [28]. In general: What is the relation between the ER-staircode and the generalized persistence diagram?

2. Extension to $d$-aug-MSs. Can we generalize our results to the setting of more than two parameters? Namely, for $d$-aug-MSs $\mathcal{X}^{d}:=\left(X, d_{X}, f_{1}, f_{2}, \ldots, f_{d}\right), f_{i}: X \rightarrow \mathbb{R}, i=1, \ldots, d$, can we recover the zeroth homological information of the $d+1$-parameter filtration induced by $\mathcal{X}^{d}$ by devising "an elder-rule-staircode" of $\mathcal{X}^{d}$ ? Note that, under the assumption the set $\left\{\left(f_{i}(x)\right)_{i=1}^{d} \in \mathbb{R}^{d}: x \in X\right\}$ is totally ordered in the poset $\mathbb{R}^{d}$, a straightforward generalization of the elder-rule staircode is conceivable. However, without this strict assumption, it is not very clear how elder-rule-staircodes should be defined. 
3. Extension to higher-order homology. The ambiguity mentioned in the previous paragraph also arises when trying to devise an "elder-rule-staircode" for higher-order homology of a multiparameter filtration; namely, when $k \geq 1$, the birth indices of $k$-cycles are not necessarily totally ordered in the multiparameter setting, and thus determining which cycle is older than another is not clear in general.

4. Metrics and stability. Recall that the collection $E(\mathcal{X})$ of all possible ER-staircodes of an aug-MS $\mathcal{X}$ is an invariant of $\mathcal{X}$ (the paragraph after Example 3.6). One possible metric between two collections of ER-staircodes is the Hausdorff distance $d_{\mathrm{H}}^{b}$ in the metric space of barcodes over $\mathbb{R}^{2}$ with the generalized bottleneck distance $d_{b}$ [5]. On the other hand, there exists a metric $d_{\mathrm{GH}}^{1}$ which measures the difference between aug-MSs [11] (see also [16]) and let $d_{\mathrm{I}}$ be the interleaving distance between 2-parameter persistence modules [32]. Are there constants $\alpha, \beta>0$ such that for all aug-MSs $\mathcal{X}$ and $\mathcal{Y}$, the inequalities below hold?

$$
\alpha \cdot d_{\mathrm{I}}\left(\mathrm{H}_{0}\left(\mathcal{R}_{\bullet}^{\mathrm{bi}}(\mathcal{X})\right), \mathrm{H}_{0}\left(\mathcal{R}_{\bullet}^{\mathrm{bi}}(\mathcal{Y})\right)\right) \leq d_{\mathrm{H}}^{b}(E(\mathcal{X}), E(\mathcal{Y})) \leq \beta \cdot d_{\mathrm{GH}}^{1}(\mathcal{X}, \mathcal{Y})
$$

5. Completeness. Recall that the collection $E(\mathcal{X})$ of all the elder-rule-staircodes of an aug-MS $\mathcal{X}$ is not a complete invariant (the paragraph after Example 3.6). How faithful is this collection in general? Is there any class of aug-MSs $\mathcal{X}$ such that $E(\mathcal{X})$ completely characterizes $\mathcal{X}$ ?

\section{A Missing details from Section 3}

Proof of Proposition 3.5. Let $x \in X$ be the point which achieves the minimum of $f_{X}$. Then $I_{x}=\left\{(\sigma, \varepsilon) \in \mathbb{R}^{2}:\left(f_{X}(x), 0\right) \leq(\sigma, \varepsilon)\right\}$, the closed quadrant whose lower-left corner is $\left(f_{X}(x), 0\right)$. Let $y \in X$ be a point which does not achieve the minimum of $f_{X}$. Define $u_{y}: \mathbb{R} \rightarrow \mathbb{R}_{\geq 0}$ by sending $\sigma \in \mathbb{R}$ to the minimum $\varepsilon \in \mathbb{R}_{\geq 0}$ for which there exists $z \in X$ with $f_{X}(z)<f_{X}(y)$ such that $y$ belongs to the same block with $z$ in the partition $\pi_{0}\left(\mathcal{R}_{\varepsilon}\left(X_{\sigma}, d_{X}\right)\right)$ (see the paragraph after Definition 4.1). It is clear that $u_{y}$ is non-increasing. Also, since $X$ is finite, $u_{y}$ is piecewise constant. By observing $I_{y}=\left\{(\sigma, \varepsilon) \in \mathbb{R}^{2}: \sigma \in\left[f_{X}(y), 0\right)\right.$ and $\varepsilon \in$ $\left.\left[0, u_{y}(\sigma)\right)\right\}$, we complete the proof.

We precisely define the $j$-th type corner points of staircase intervals depicted in Figure 3:

Definition A.1 (Types of corner points). Let $I$ be a staircase interval of $\mathbb{R}^{2}$. Fix $\mathbf{a} \in \mathbb{R}^{2}$. This a is a 0-th type corner point of $I$ if

$$
\mathbb{1}_{I}(\mathbf{a})=1, \quad \lim _{\varepsilon \rightarrow 0+} \mathbb{1}_{I}(\mathbf{a}-(\varepsilon, 0))=\lim _{\varepsilon \rightarrow 0+} \mathbb{1}_{I}(\mathbf{a}-(0, \varepsilon))=\lim _{\varepsilon \rightarrow 0+} \mathbb{1}_{I}(\mathbf{a}-(\varepsilon, \varepsilon))=0 .
$$

The point $\mathbf{a}$ is a 1-st type corner point of $I$ if

$$
\mathbb{1}_{I}(\mathbf{a})-\lim _{\varepsilon \rightarrow 0+} \mathbb{1}_{I}(\mathbf{a}-(\varepsilon, 0))-\lim _{\varepsilon \rightarrow 0+} \mathbb{1}_{I}(\mathbf{a}-(0, \varepsilon))+\lim _{\varepsilon \rightarrow 0+} \mathbb{1}_{I}(\mathbf{a}-(\varepsilon, \varepsilon))=-1 .
$$

The point a is a 2-nd type corner point of $I$ if

$$
\mathbb{1}_{I}(\mathbf{a})=0, \quad \lim _{\varepsilon \rightarrow 0+} \mathbb{1}_{I}(\mathbf{a}-(\varepsilon, 0))=\lim _{\varepsilon \rightarrow 0+} \mathbb{1}_{I}(\mathbf{a}-(0, \varepsilon))=\lim _{\varepsilon \rightarrow 0+} \mathbb{1}_{I}(\mathbf{a}-(\varepsilon, \varepsilon))=1 .
$$

We remark that Definition A.1 is closely related to the differential of an interval introduced in [23]. 


\section{B Missing details from Section 4}

In order to show that Definition 4.3 is well-defined, it suffices to show:

Proposition B.1 (Elder-rule-barcode is well-defined). Let $\theta_{X}: \mathbb{R} \rightarrow \operatorname{Subpart}(X)$ be $a$ treegram over $X$ and suppose that there exist different $y, z \in X$ with $b(y)=b(z)$. Consider two orders $<_{1},<_{2}$ which are the same except for the pair $y, z$, i.e. $y<_{1} z$ and $z<_{2} y$. Then, $\left\{\left[b(x), d^{<1}(x)\right): x \in X\right\}=\left\{\left\{b(x), d^{<2}(x)\right): x \in X\right\}$.

Proof. For $x \in X$ different from $y$ and $z$, it is clear that $\left[b(x), d^{<_{1}}(x)\right)=\left[b(x), d^{<2}(x)\right)$. Hence, letting $b:=b(y)=b(z)$, it suffices to show that

$$
\left\{\left\{b, d^{<1}(y)\right),\left[b, d^{<1}(z)\right)\right\}=\left\{\left\{\left[b, d^{<2}(y)\right),\left[b, d^{<2}(z)\right)\right\}\right\},
$$

or equivalently $\left\{\left\{d^{<_{1}}(y), d^{<_{1}}(z)\right\}=\left\{\left\{d^{<_{2}}(y), d^{<_{2}}(z)\right\}\right.\right.$. Assume that $y$ and $z$ merge at $\varepsilon=r_{0}$ in $\theta_{X}$. Since $<_{1}$ and $<_{2}$ are the same except for the pair $y, z$, we use $<$ to denote both $<_{1}$ and $<_{2}$ when we compare $y, z$ with the other elements in $X$. In the treegram $\theta_{X}$, there are only two possible cases: (Case 1) An element $w \in X$ with $w<y$, $z$ merges with the block containing both $y, z$ at $\varepsilon=r_{1} \geq r_{0}$. Then, $d^{<_{1}}(y)=r_{1}$ and $d^{<_{1}}(z)=r_{0}$, whereas $d^{<_{2}}(y)=r_{0}$ and $d^{<2}(z)=r_{1}$. (Case 2) assume that there are $w_{1}<y$ and $w_{2}<z$ such that $w_{1}$ and $y$ merge at $\varepsilon=r_{2} \leq r_{0}$ and $w_{2}$ and $z$ merge at $\varepsilon=r_{3} \leq r_{0}$ (it is possible that $w_{1}=w_{2}$ ). Then, $d^{<1}(y)=d^{<2}(y)=r_{2}$ and $d^{<1}(z)=d^{<2}(z)=r_{3}$, completing the proof.

\section{C $\quad$ Missing details from Section 6}

\section{C.1 Proofs of Theorem 6.1}

We first present a lemma needed for the proof of Theorem 6.1 (a). For simplicity, we assume that all distances between points in $X$ (and thus edge weights in $G_{i} \mathrm{~s}$ ) are distinct. If this is not the case, we only need to fix a total order compatible with all distances for the algorithm to work in the same way.

Lemma C.1. Given a graph $G=(V, E)$ with distinct edge weights, if $e \in E$ is the largest edge of a cycle $C$ in $G$, then e will not appear in the $M S T$ of $G$.

Proof. Let us denote $e$ as the largest edge in the cycle $C$ of size $k+1$ where $C$ consists of edges $e, e_{1}, e_{2}, \ldots, e_{k}$. Also denote the MST of $G$ as $T$. From $C$ and $T$, We will give a way to construct new cycle $C^{\prime}$ where all edges except $e$ belong to $T$.

Since $T$ is MST, for any $i \in\{1,2, \ldots, k\}$, if $e_{i}$ does not belong to $T$, adding $e_{i}$ will form a cycle $C_{i}$ where $e_{i}$ in the largest edge and the only non-MST edge in $C_{i}$.

Construct new cycle $C^{\prime}=C+\Sigma_{i \in\left\{j \mid e_{j} \notin T\right\}} C_{i}$ where the addition is performed on $\mathbb{F}_{2}$. Every time we add $C_{i}$, it will cancel out $e_{i}$. Since we did for all non-MST edges, the resulting cycle $C^{\prime}$ will consist of all MST edges plus $e$.

We argue that $e$ is also the largest edge in $C^{\prime}$. This holds because every time we add $C_{i}$, we knew $e_{i}$ is the largest edge in $C_{i}$, and because $|w(e)| \geq\left|w\left(e_{i}\right)\right|$ where $w$ is weight function on edges, we knew $e$ is also the largest edge in $C^{\prime}$. By the property of MST (any non-MST edge is the largest edge in the cycle created by adding itself to MST.), we conclude that $e$ is a non-MST edge. 


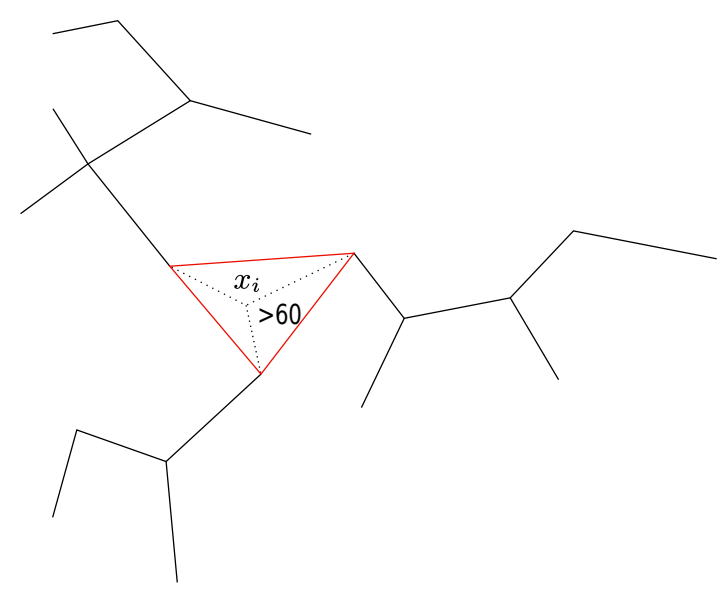

Figure 14: Illustration of packing argument in Theorem 6.1. $x_{i}$ is the new vertex. Dashed edges are new edges entering $T_{i}$. Red edges are non-MST edges and therefore by the property of non-MST edges, angle corresponding to red edges must be at least $\frac{\pi}{3}$.

The following lemma, combined with the argument in the main text, will establish the time complexity of the algorithm to compute ER-staircode for the case when $X$ is from a fixed dimensional Euclidean space $\mathbb{R}^{d}$.

Lemma C.2. Let $T_{i-1}$ and $T_{i}$ be the $M S T$ of $G_{i-1}$ and $G_{i}$ as defined in the algorithm. For fixed dimensional $\mathbb{R}^{d}$ and $d_{X}$ to be Euclidean distance, the number of edges in $T_{i} \backslash T_{i-1}$ is $O(1)$ (depending on $d$ ).

Proof. Recall that $G_{i}$ is obtained by adding a new vertex $x_{i}$ and edges incident to $x_{i}$. First, note that by Lemma C.1, edges in $T_{i}=M S T\left(G_{i}\right)$ are either from $T_{i-1}=M S T\left(G_{i-1}\right)$, or new edges just inserted. That is, no edge in $G_{i-1} \backslash T_{i-1}$ will contribute to $T_{i}$ : This is such an edge will be the largest-weight edge of some cycle in $G_{i}$.

We now prove that for the case where $X \subset \mathbb{R}^{d}$ only $O(1)$ (where the big-O hides terms depending on $d$ ) new edges (incident to $x_{i}$ ) can be in $T_{i}$.

In particular, comparing $T_{i-1}$ and $T_{i}$, there are only two types of edges that are subject to change: 1) edges that are in $T_{i-1}$ but will leave $T_{i-1}$ and 2) edges incident to $x_{i}$ and will enter the new $T_{i}$.

Assume there are $k$ edges that will leave $T_{i-1}$. By deleting them, the original $T_{i-1}$ is decomposed into $k+1$ small trees. There must be $k+1$ edges incident to $x_{i}$ entering $T_{i}$. We denote those $k+1$ edges as $\mathcal{E}_{\text {new }, i}=\left\{x_{i} x_{i_{1}}, x_{i} x_{i_{2}}, \ldots, x_{i} x_{i_{k+1}}\right\}$

Pick any two nodes $a, b$ from $\mathcal{E}_{\text {new }, i}=\left\{x_{i_{1}}, x_{i_{2}}, \ldots, x_{i_{k+1}}\right\}$, they will from a triangle with $x_{i}$. By property of MST, edge $a b$ in triangle $\triangle_{a b x_{i}}$ is the longest edge as $a b \notin T_{i}$ while $x_{i} a, x_{i} b \in T_{i}$. By elementary Euclidean geometry, it can be shown that angle $\varangle a x_{i} b$ must be no less than $\frac{\pi}{3}$, and this holds for every pair of nodes from $\mathcal{E}_{\text {new }, i}=\left\{x_{i_{1}}, x_{i_{2}}, \ldots, x_{i_{k+1}}\right\}$. Now by a packing argument, we can show that there can be $O\left(C^{d}\right)$ such well-separated points around $x_{i}$ in $\mathbb{R}^{d}$ for some constant $C$.

Indeed, consider the unit sphere $S$ around $x_{i}$ in $\mathbb{R}^{d}$, and let $y_{j}$ be the intersection of the ray starting at $x_{i}$ and passing through $x_{i_{j}}$ with $S$. The previous paragraph establishes that the angle $\varangle y_{j} x_{i} y_{j^{\prime}} \geq \pi / 3$ for any $j \neq j^{\prime} \in[1, k+1]$. It then follows that the geodesic distance 


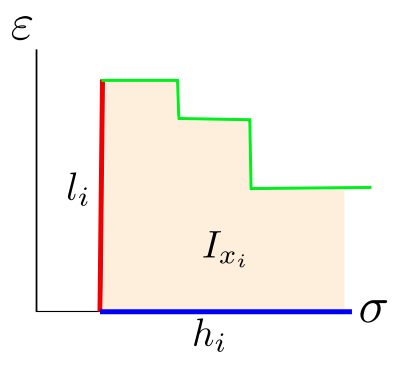

$(A)$

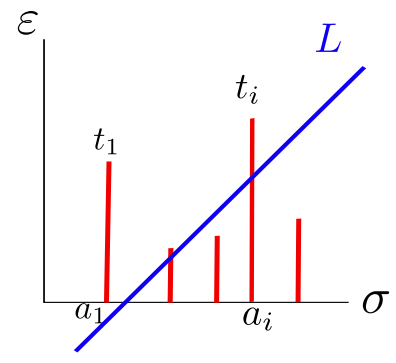

$(B)$

Figure 15: (A) An illustration of $I_{x_{i}}$ where its lower envelope $l_{i}$ (vertical line segment) and $h_{i}$ (horizontal ray) are colored red and blue. (B) An illustration of Case 2 in Lemma C.4

between $y_{j}$ and $y_{j^{\prime}}$ on $S$ is at least $\pi / 3$. In other words, geodesic balls of radius $\pi / 6$ centered at $y_{j}$ 's for $j \in[1, k+1]$ have to be all disjoint. The number of such balls (and thus $k+1$ ) is at most $\operatorname{Area}(S) / B$ where $\operatorname{Area}(S)$ stands for the surface volume of unit $d$-sphere in $\mathbb{R}^{d}$, while $B$ is the volume of a $(d-1)$-ball of radius $\sin \frac{\pi}{6}=\frac{1}{2}$. Hence there exists some constant $C>1$ such that $k=O\left(C^{d}\right)$. This proves the lemma.

We now present proofs for part (b) and (c) of Theorem 6.1.

Lemma C.3. The size of ER-staircode is $O\left(n^{2}\right)$.

Proof. We claim that for every $x \in \mathcal{X}$, the size of $I_{x}$ is $O(n)$ and the lemma will then follow. This holds because every $I_{x}$ has a staircase shape, and the $x$-coordinates of corner points can only be one of the values $f_{X}\left(x_{i}\right)$ for some $x_{i} \in X$.

Lemma C.4. Given $I_{\mathcal{X}}$, after $O\left(n^{2} \log n\right)$ time preprocessing, we can build a data structure of size $O\left(n^{2}\right)$ so that, given any line $L$ with positive slope, the zeroth fibered barcode $B^{L}$ w.r.t. $L$ can be computed in $O\left(\left|B^{L}\right| \log n\right)$ time where $\left|B^{L}\right|$ is the size of fibered barcode.

Proof. First, given an $I_{x}$, recall that it has a staircase shape: see Figure 15. In particular, its lower envelop consists of one vertical and one horizontal segment. Its upper envelope $U$ is the graph of a piecewise constant non-decreasing function in the plane consisting of $O(n)$ horizontal and vertical line segments. Given a line $L$ with positive slope, its intersection with the lower envelop of $I_{x}$ thus takes only $O(1)$ time. The upper envelope can only intersect with $L$ at most one point, either within some horizontal segment of $U$ or within a vertical segment of $U$. To identify this intersection point, we simply binary search twice: once among all horizontal segments, and once among all vertical segments, in $O(\log n)$ time.

Next, we show that we can avoid checking all $n$ number of $I_{x}$ s. Instead, we will compute only the set $\widehat{\mathcal{I}}_{L}$ of $I_{x}$ s that will intersect $L$ : Note that there are $k=\left|B^{L}\right|$ number of such staircodes. In what follows, we describe how to preprocess all staircodes so that this set $\widehat{\mathcal{I}}_{L}$ can be reported in $O(\log n+k)$ time.

Specifically, for any $x_{i} \in \mathcal{X}$, let $\ell_{i}$ and $h_{i}$ be the vertical and horizontal segments of the lower-envelop of $I_{x_{i}}$ - see Figure 15 for an illustration. Note that each $h_{i}$ is in fact a half line in the $x$-axis. It is easy to see that the line $L$ intersects $I_{x_{i}}$ if and only if $L$ intersects either $\ell_{i}$ or $h_{i}$. 
Case 1: reporting intersection with $h_{i} \mathrm{~s}$. Given the collection of all $h_{i} \mathrm{~s}, i \in[1, n]$, in $O(n \log n)$ time, we can build a standard 1D range reporting data structure of size $O(n)$, over the collection of left endpoints $a_{i}$ 's of $\left.h_{i} \mathrm{~s}, i \in[1, n]\right\}$, so that given a query point $b$, we can report all points in $\left\{a_{i}\right\}$ to the left of $b$, in $O(\log n+s)$ time where $s$ is the number of such points.

Now given a query line $L$, let $b_{L}$ be the intersection between $L$ and the $x$-axis. We use the data strutcure to compute, say $k_{1}$ number of points from $\left\{a_{i}\right\}$ to the left of $b_{L}$, in $O\left(\log n+k_{1}\right)$ time. Each such point corresponds to a ray $h_{i}$ that will intersect $L$.

Case 2: reporting intersection with $\ell_{i} \mathrm{~s}$. What remains is to build a data structure to report the set of $\ell_{i}$ s intersecting $L$. To this end, note that for each $i \in[1, n]$, the point $a_{i}$ introduced above is also the bottom endpoint of the vertical segment $\ell_{i}$; let $t_{i}$ denote the top endpoint for $\ell_{i}$. Given a query line $L$, we wish to report all $i$ 's such that $t_{i}$ is above $L$ while $a_{i}$ is below $L$. Again, let $b_{L}$ denote the intersection of $L$ with the $x$-axis: As the slope of $L$ is positive, if a vertical segment $\ell_{i}$ intersects $L$, then $a_{i}$ must lie to the right of $b_{L}$.

Now for each $j \in[1, n]$, set

$$
A_{j}:=\left\{t_{i} \mid a_{i} \geq a_{j}\right\} .
$$

Given $L$, let $a_{r}$ be the closest point to $b_{L}$ with $a_{r} \geq b_{L}$. Obviously, the line $L$ intersects $\ell_{i}$ if and only if $t_{i} \in A_{r}$ and $t_{i}$ is above $L$. Hence we want to perform a halfplane range reporting query among the points in $A_{r}$. To this end, for each $i \in[1, n]$, we use the classic approach of [18] to build a data structure of size $O\left(\left|A_{i}\right|\right)=O(n)$ in time $O\left(\left|A_{i}\right| \log \left|A_{i}\right|\right)=O(n \log n)$, so that given a line $L$, the set of points from $A_{i}$ above $L$ can be reported in $O(\log n+s)$ time where $s$ is the number of such points. Overall, the total size of all such data structures for all $i \in[1, n]$ is $O\left(n^{2}\right)$ and can be constructed in $O\left(n^{2} \log n\right)$ time. Given $L$, we first identify $a_{r}$ as described above, and then query for the set of $t_{i}$ s from $A_{r}$ lying above $L$ in $O\left(\log n+k_{2}\right)$ time, where $k_{2}$ is the number of such $t_{i}$ s.

Putting Case 1 and Case 2 together, we can report all $k=k_{1}+k_{2}$ staircodes $\widehat{\mathcal{I}}_{L}$ intersecting a query line $L$ of positive slope in time $O(\log n+k)$ time.

Once we have $\widehat{\mathcal{I}}_{L}$, for each $I_{x} \in \widehat{\mathcal{I}}_{L}$, we use the procedure described at the beginning of this proof to compute the intersection between $L$ and $I_{x}$ in $O(\log n)$ time for each $I_{x}$. In total, it takes $O(k \log n)$ to compute all intersections. The total query time is $O(\log n+k+k \log n)=$ $O(k \log n)=O\left(\left|B^{L}\right| \log n\right)$ as claimed.

Lemma C.5. Given $I_{\mathcal{X}}$, we can compute the zeroth graded Betti numbers in $O\left(n^{2}\right)$ time.

Proof. Since the total number of segments of ER-staircode is $O\left(n^{2}\right)$ so is the number of corner points. In other words, only $O\left(n^{2}\right)$ grades could potentially have a non-zero $\gamma_{i}^{\mathcal{X}}$ or $\beta_{i}^{M}$ value, for $i=0,1$, or 2 . We can therefore compute graded Betti numbers according to the formula in Section 5.4, by evaluating $\gamma_{i}^{\mathcal{X}}$ and $\beta_{i}^{M}$ at each of the $O\left(n^{2}\right)$ possible grades.

\section{C.2 Proof of Lemma 6.2}

We now give a detailed description of the process to recover ER-staircode from the collection of treegrams in $O\left(n^{2}\right)$ time. Recall conqueror is defined in Section 4.2. When $x^{\prime}$ is a conqueror of $x$ in $G_{i}=G_{\sigma_{i}}$, we also say $x$ is conquered by $x^{\prime}$ at height $u_{X_{\sigma_{i}}}\left(x, x^{\prime}\right)$. To 

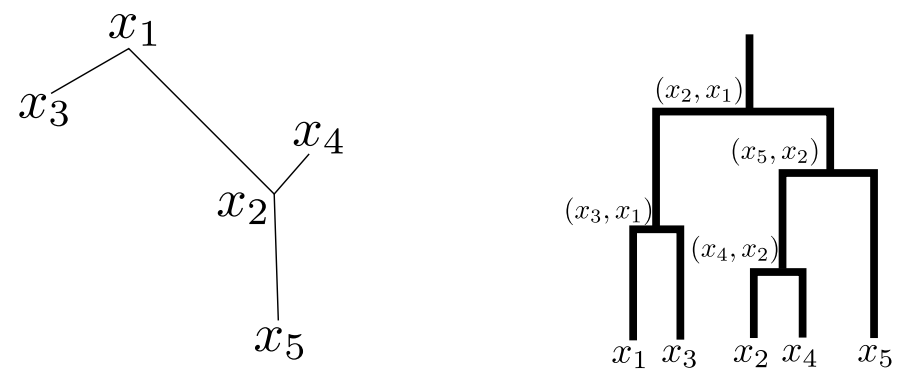

Figure 16: The left figure shows a MST of 5 points where node with low(high) index appear early(later). The weight of each edge is the length of the edge (e.g, $\left.w\left(x_{1}, x_{3}\right)>w\left(x_{2}, x_{4}\right)\right)$. The right figure shows the corresponding decorated treegram. At each non-leaf node, we only need to store a tuple where the first number stands for the index of the node that is conquered while the second number stands for the index of the node that has not been conquered (eldest) in the subtree.

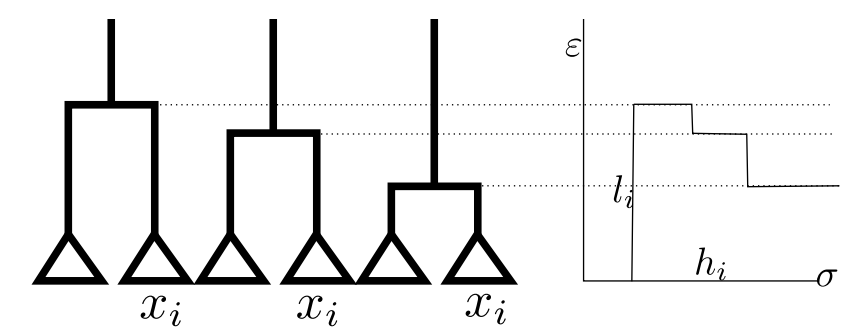

Figure 17: Illustration of assembling process to recover $I_{x_{i}}$. Note that we do not plot the whole treegram at each function value for simplicity. $x_{i}$ here is a leaf in the right subtree of every treegram. We will first compute the decorated treegrams, illustrated in Figure 16. From these decorated treegrams, we are able to retrieve $\epsilon$ values of $I_{x}$ at for each of the $n$ function values $\sigma_{1}, \ldots, \sigma_{n}$ with $\sigma_{i}=f\left(x_{i}\right)$ and thus assemble $I_{x_{i}}$. 
convert treegrams at different function values to staircode, we will decorate the treegrams with some extra information. On the high level, we need to keep the information about the node index conquered at different heights in the treegram, which can be done in linear time by traversing the treegram from bottom to top.

Specifically, denote the sorted height values of treegram $\theta_{i}$ at function value $\sigma_{i}$ as $\mathcal{E}_{i}=$ $\left\{\epsilon_{1}<\epsilon_{2}<\ldots, \epsilon_{i-1}\right\}$. At each non-leaf node of height $\epsilon_{j} \in \mathcal{E}_{i}$ in the treegram $\theta_{i}$, we record a) the index of the node that is conquered at height $\epsilon_{j}$ and b) index of the single node in subtrees (rooted at height $\epsilon_{j}$ ) who has not been conquered yet. b) is needed to update a) of the node at height $\epsilon_{j+1}$ in constant time. Traversing treegrams bottom-up and compute a) and b) for every non-rooted node takes $O(i)=O(n)$ time. An illustration of the idea of decorated treegrams is shown in Figure 16.

After computing $n$ decorated treegrams at $n$ function values, we can recover ER-staircode by assembling decorated treegrams in the following way. Without loss of generality, we state the process to recover single $I_{x}$ in ER-staircode. For every function value $\epsilon_{i}$, find corresponding $\sigma$ (i.e., $\left.u_{X_{\sigma_{i}}}\left(x, x^{\prime}\right)\right)$ in $\mathcal{E}_{i}$ at which $x$ is conquered. Repeat this process for all function values will recover $I_{x}$. Figure 17 illustrates the idea.

We restate the Lemma 6.2 with a proof.

Lemma C.6. A decorated ER-staircode for the finite aug-MS $\left(X, d_{X}, f_{X}\right)$ can be computed from the collection of dendrograms $\left\{\theta_{i}, i \in[1, n]\right\}$ in $O\left(n^{2}\right)$ time.

Proof. The decoration of every treegrams takes $O(n)$ time and in total $O\left(n^{2}\right)$ for $n$ treegrams. Assembling $I_{x}$ for each $x \in \mathcal{X}$ takes $O(n)$ time since the complexity of every $I_{x}$ is $O(n)$ so in total recovering ER-staircode takes $O\left(n^{2}\right)$ time. For the correctness, we prove our process can recover $I_{x}$ for every $x \in \mathcal{X}$. This holds because for any $x \in \mathcal{X}$ and $\sigma_{i} \in f_{X}$ we can recover $u_{X_{\sigma_{i}}}\left(x, x^{\prime}\right)$ where $x^{\prime}$ is the conqueror of $x$.

\section{References}

[1] Hideto Asashiba, Mickaël Buchet, Emerson G Escolar, Ken Nakashima, and Michio Yoshiwaki. On interval decomposability of $2 \mathrm{D}$ persistence modules. arXiv preprint arXiv:1812.05261, 2018.

[2] Hideto Asashiba, Emerson G Escolar, Ken Nakashima, and Michio Yoshiwaki. On approximation of $2 \mathrm{D}$ persistence modules by interval-decomposables. arXiv preprint arXiv:1911.01637, 2019.

[3] Gorô Azumaya et al. Corrections and supplementaries to my paper concerning krullremak-schmidt's theorem. Nagoya Mathematical Journal, 1:117-124, 1950.

[4] Ulrich Bauer, Magnus B Botnan, Steffen Oppermann, and Johan Steen. Cotorsion torsion triples and the representation theory of filtered hierarchical clustering. arXiv preprint arXiv:1904.07322, 2019.

[5] Magnus Botnan and Michael Lesnick. Algebraic stability of zigzag persistence modules. Algebraic \&f geometric topology, 18(6):3133-3204, 2018. 
[6] Peter A Brooksbank and Eugene M Luks. Testing isomorphism of modules. Journal of Algebra, 320(11):4020-4029, 2008.

[7] Chen Cai and Yusu Wang. Understanding the power of persistence pairing via permutation test. arXiv preprint arXiv:2001.06058, 2020.

[8] Ricardo JGB Campello, Davoud Moulavi, and Jörg Sander. Density-based clustering based on hierarchical density estimates. In Pacific-Asia conference on knowledge discovery and data mining, pages 160-172. Springer, 2013.

[9] G. Carlsson and A. Zomorodian. The theory of multidimensional persistence. Discrete \&6 Computational Geometry, 42(1):71-93, 2009.

[10] Gunnar Carlsson and Facundo Mémoli. Characterization, stability and convergence of hierarchical clustering methods. Journal of machine learning research, 11(Apr):14251470, 2010.

[11] Gunnar Carlsson and Facundo Mémoli. Multiparameter hierarchical clustering methods. In Classification as a Tool for Research, pages 63-70. Springer, 2010.

[12] Gunnar Carlsson and Afra Zomorodian. The theory of multidimensional persistence. Discrete \& Computational Geometry, 42(1):71-93, 2009.

[13] Mathieu Carriere, Frédéric Chazal, Yuichi Ike, Théo Lacombe, Martin Royer, and Yuhei Umeda. A general neural network architecture for persistence diagrams and graph classification. arXiv preprint arXiv:1904.09378, 2019.

[14] Andrea Cerri, Barbara Di Fabio, Massimo Ferri, Patrizio Frosini, and Claudia Landi. Betti numbers in multidimensional persistent homology are stable functions. Mathematical Methods in the Applied Sciences, 36(12):1543-1557, 2013.

[15] Wojciech Chacholski, Martina Scolamiero, and Francesco Vaccarino. Combinatorial presentation of multidimensional persistent homology. Journal of Pure and Applied Algebra, 221(5):1055-1075, 2017.

[16] Frédéric Chazal, David Cohen-Steiner, Leonidas J Guibas, Facundo Mémoli, and Steve Y Oudot. Gromov-Hausdorff stable signatures for shapes using persistence. In Computer Graphics Forum, volume 28 (5), pages 1393-1403. Wiley Online Library, 2009.

[17] Frédéric Chazal, Leonidas J Guibas, Steve Y Oudot, and Primoz Skraba. Scalar field analysis over point cloud data. Discrete \& Computational Geometry, 46(4):743, 2011.

[18] Bernard Chazelle, Leo J. Guibas, and D. T. Lee. The power of geometric duality. BIT Numerical Mathematics, 25(1):76-90, Mar 1985.

[19] Francis Chin and David Houck. Algorithms for updating minimal spanning trees. Journal of Computer and System Sciences, 16(3):333-344, 1978. 
[20] David Cohen-Steiner, Herbert Edelsbrunner, and John Harer. Stability of persistence diagrams. Discrete \& Computational Geometry, 37(1):103-120, 2007.

[21] Justin Curry. The fiber of the persistence map for functions on the interval. Journal of Applied and Computational Topology, 2(3-4):301-321, 2018.

[22] Tamal K Dey and Cheng Xin. Computing bottleneck distance for 2-d interval decomposable modules. In Proceedings of the thirty-fourth International Symposium on Computational Geometry (SoCG 2018), pages 32:1-32:15, 2018.

[23] Tamal K Dey and Cheng Xin. Generalized persistence algorithm for decomposing multiparameter persistence modules. arXiv preprint arXiv:1904.03766, 2019.

[24] Herbert Edelsbrunner and John Harer. Computational topology: an introduction. American Mathematical Soc., 2010.

[25] David Eisenbud. Commutative Algebra: with a view toward algebraic geometry, volume 150. Springer Science \& Business Media, 2013.

[26] Heather A Harrington, Nina Otter, Hal Schenck, and Ulrike Tillmann. Stratifying multiparameter persistent homology. SIAM Journal on Applied Algebra and Geometry, 3(3):439-471, 2019.

[27] Christoph Hofer, Roland Kwitt, Marc Niethammer, and Andreas Uhl. Deep learning with topological signatures. In Advances in Neural Information Processing Systems, pages 1634-1644, 2017.

[28] Woojin Kim and Facundo Mémoli. Generalized persistence diagrams for persistence modules over posets. arXiv preprint arXiv:1810.11517, 2018.

[29] Woojin Kim and Facundo Mémoli. Spatiotemporal persistent homology for dynamic metric spaces. Discrete $\&$ Computational Geometry, pages 1-45, 2020.

[30] Kevin P. Knudson. A refinement of multi-dimensional persistence. Homology, Homotopy and Applications, 10(1):259-281, 2008.

[31] Claudia Landi. The rank invariant stability via interleavings. In Research in Computational Topology, pages 1-10. Springer, 2018.

[32] Michael Lesnick. The theory of the interleaving distance on multidimensional persistence modules. Found. Comput. Math., 15(3):613-650, June 2015.

[33] Michael Lesnick and Matthew Wright. Interactive visualization of 2-d persistence modules. arXiv preprint arXiv:1512.00180, 2015.

[34] Michael Lesnick and Matthew Wright. Computing minimal presentations and betti numbers of 2-parameter persistent homology. arXiv preprint arXiv:1902.05708, 2019.

[35] Álvaro Martínez-Pérez. A density-sensitive hierarchical clustering method. Journal of Classification, 35(3):481-510, 2018. 
[36] Alex McCleary and Amit Patel. Multiparameter persistence diagrams. arXiv preprint arXiv:1905.13220v3, 2019.

[37] Ezra Miller. Data structures for real multiparameter persistence modules. arXiv preprint arXiv:1709.08155, 2017.

[38] James R Munkres. Elements of algebraic topology. Addison-Wesley Menlo Park, 1984.

[39] Amit Patel. Generalized persistence diagrams. Journal of Applied and Computational Topology, 1(3-4):397-419, 2018.

[40] Irena Peeva. Graded syzygies, volume 14. Springer Science \& Business Media, 2010.

[41] Zane Smith, Samir Chowdhury, and Facundo Mémoli. Hierarchical representations of network data with optimal distortion bounds. In 2016 50th Asilomar Conference on Signals, Systems and Computers, pages 1834-1838. IEEE, 2016.

[42] Oliver Vipond. Multiparameter persistence landscapes. Journal of Machine Learning Research, 21(61):1-38, 2020.

[43] Qi Zhao and Yusu Wang. Learning metrics for persistence-based summaries and applications for graph classification. In 33rd Annu. Conf. Neural Inf. Processing Systems (NeuRIPS), 2019. to appear.

[44] Afra Zomorodian and Gunnar Carlsson. Computing persistent homology. Discrete 85 Computational Geometry, 33(2):249-274, 2005. 